\title{
Numerical Model of an Evaporating Thin Film
}

by

\author{
John L. Polansky
}

\author{
A Thesis submitted to \\ the Faculty of Graduate Studies and Research \\ in partial fulfilment of \\ the requirements for the degree of \\ Master of Applied Science
}

Ottawa-Carleton Institute for

Mechanical and Aerospace Engineering

Department of Mechanical and Aerospace Engineering

Carleton University

Ottawa, Ontario, Canada

April 2011

Copyright (C)

2011 - John L. Polansky 


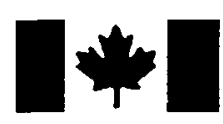

\author{
Library and Archives \\ Canada \\ Published Heritage \\ Branch \\ 395 Wellington Street \\ Ottawa ON K1A ON4 \\ Canada
}

Bibliothèque et

Archives Canada

Direction du

Patrimoine de l'édition

395, rue Wellington

Ottawa ON K1A ON4

Canada
Your file Votre référence
ISBN: $978-0-494-81670-7$
Our file Notre référence
ISBN: $978-0-494-81670-7$
NOTICE:

The author has granted a nonexclusive license allowing Library and Archives Canada to reproduce, publish, archive, preserve, conserve, communicate to the public by telecommunication or on the Internet, loan, distribute and sell theses worldwide, for commercial or noncommercial purposes, in microform, paper, electronic and/or any other formats.

The author retains copyright ownership and moral rights in this thesis. Neither the thesis nor substantial extracts from it may be printed or otherwise reproduced without the author's permission.
AVIS:

L'auteur a accordé une licence non exclusive permettant à la Bibliothèque et Archives Canada de reproduire, publier, archiver, sauvegarder, conserver, transmettre au public par télécommunication ou par l'Internet, prêter, distribuer et vendre des thèses partout dans le monde, à des fins commerciales ou autres, sur support microforme, papier, électronique etlou autres formats.

L'auteur conserve la propriété du droit d'auteur et des droits moraux qui protège cette thèse. $\mathrm{Ni}$ la thèse ni des extraits substantiels de celle-ci ne doivent être imprimés ou autrement reproduits sans son autorisation.
In compliance with the Canadian Privacy Act some supporting forms may have been removed from this thesis.

While these forms may be included in the document page count, their removal does not represent any loss of content from the thesis.
Conformément à la loi canadienne sur la protection de la vie privée, quelques formulaires secondaires ont été enlevés de cette thèse.

Bien que ces formulaires aient inclus dans la pagination, il n'y aura aucun contenu manquant. 
The undersigned recommend to the Faculty of Graduate Studies and Research acceptance of the Thesis

\title{
Numerical Model of an Evaporating Thin Film
}

\author{
Submitted by John L. Polansky \\ in partial fulfilment of the requirements for the degree of \\ Master of Applied Science
}

T. Kaya, Supervisor

M. Yaras, Department Chair

Carleton University

2011 


\section{Abstract}

A mathematical model is developed here in, to describe the thermodynamics of an evaporating thin film. The model is derived such that the working fluid is that of a non-polar nature. The evaporating thin film is described by a highly non-linear third order differential equation. It was solved numerically through a form of RungeKutta solver written in MATLAB. The solutions were found to be very sensitive to the choice of the initial conditions. This added to the investigation of perturbation sensitivities, where it was found that the thickness and slope terms of the differential equation are the most sensitive to perturbations.

A range of different simulations were performed to determine how the thin film was affected by the adjustment of different parameters. For the Marangoni term, it was found to have little to no effect on the thin film profile, given the low superheat. For the wall slip condition, it was found to lengthen the thin film region with an increase in wall slip length. For the case of different fluids, the MATLAB code successfully illustrated the effects of different operating fluids on the evaporative characteristics.

The last two cases were for that of the channel width and superheat ranges. The channel width was found to increase the thin film length and evaporative mass flux capability with an associated increase in channel width. Last to be considered was the variance of the applied superheat. The results of the study indicate that an increase in applied superheat results in the shortening of the thin film and the increase in evaporative mass flux. 
To my father. 


\section{Acknowledgments}

I would like to thank my supervisor, Professor Tarik Kaya, for his guidance and support throughout the course of my research. It is with his support that I was able to conduct my research with both direction and purpose.

I would also like to thank my father, Steve and brother, William for their unwaivering support in my studies. As my ambitions have taken me forward, I have never forgot where I have come from.

Lastly, I would like to extend thanks to my friends and colleagues for their encouragement and moral support. The grounded foundation for which you have provided has helped me stay a course. 


\section{Preface}

The process of evaporation has been used for centuries as a means of efficiently and effectively removing heat. Whether it is from that of hot iron or solar radiation, the ability to control and manipulate the transfer of heat drives the modern world. This drive toward greater thermal efficiency pushes engineers and scientists alike to find new and creative solutions. It is for these reasons that the study of evaporating thin films is investigated here.

The thin film, is taken to be a portion of a meniscus where the disjoining and capillary pressures dominate. As the region is sufficiently small, it can not be easily observed and requires the concurrent development of models. It is with these models that the heat transfer capacity can be estimated for the purposes of sizing and safety.

This thesis is intended to present a clear and concise model for that of a stable evaporating thin film. It is hoped that the mathematical model developed here, will provide engineers with a tool to further enhance the efficiency of heat exchanging devices, such as heat pipes. For the researchers continuing in this field, it may provide a stepping stone from which more accurate models may be devised. 


\section{Table of Contents}

Abstract $\quad$ iii

Acknowledgments $\quad$ v

$\begin{array}{lll}\text { Preface } & \text { vi }\end{array}$

Table of Contents $\quad$ vii

List of Tables $\quad$ xii

List of Figures $\quad$ xiii

Nomenclature $\quad$ xvi

1 Introduction $\quad 1$

1.1 The thin film region ........................ 2

1.2 Research motivation .................. 4

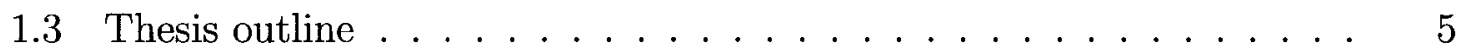

2 Literature Review $\quad 6$

2.1 The meniscus ........................... 6

2.1.1 Adsorbed region . . . . . . . . . . . . . . . 7

2.1.2 Thin film region ................. 8

2.1.3 Bulk meniscus region . . . . . . . . ....... 8 
2.2 Thin film applications $\ldots \ldots \ldots \ldots$

2.3 Model development . . . . . . . . . . . . . . . . . 9

2.3 .1 Young-Laplace . . . . . . . . . . . . . . . . . 10

2.3 .2 Disjoining pressure . . . . . . . . . . . . 11

2.3 .3 Hamaker constant. . . . . . . . . . . . . . . . 13

2.3.4 Augmented Young-Laplace . . . . . . . . . . . . . . . . 14

2.3 .5 Evaporation model . . . . . . . . . . . . . . . . . 14

2.4 Numerical studies . . . . . . . . . . . . . . . . . . . . . 15

2.4 .1 Types of numerical results $\ldots \ldots \ldots \ldots$

2.4 .2 Methods of solution . . . . . . . . . . . 16

2.4 .3 Equation behaviour . . . . . . . . . . . . . . 17

2.4 .4 Trends in numerical results . . . . . . . . . . . . 18

2.5 Alternate models . . . . . . . . . . . . . . . . . . . 19

2.6 Experimental observations . . . . . . . . . . . . . . 20

2.7 Summary and objectives . . . . . . . . . . . . . . 21

3 Mathematical Model 23

3.1 Assumptions . . . . . . . . . . . . . . . . . 25

3.2 Force balance . . . . . . . . . . . . . . . . 26

3.3 Navier-Stokes equations $\ldots \ldots \ldots \ldots \ldots \ldots$

$3.3 .1 \quad$ Assumptions . . . . . . . . . . . . . . . . . 27

3.3 .2 Boundary conditions . . . . . . . . . . . . . 28

3.4 Continuity equation . . . . . . . . . . . . . . . 28

3.5 Energy equation . . . . . . . . . . . . . . . . . 29

3.5 .1 Assumptions . . . . . . . . . . . . . . . . 29

3.5 .2 Boundary conditions $\ldots \ldots \ldots \ldots \ldots$

3.6 Liquid pressure gradient $\ldots \ldots \ldots \ldots \ldots \ldots$ 
3.7 Kelvin-Clapeyron equation . . . . . . . . . . . . . . . . . . 31

3.8 Interface temperature gradient . . . . . . . . . . . . . . 32

3.9 Complete Model . . . . . . . . . . . . . . . . . . . . . . . . . . . 32

3.10 Initial conditions . . . . . . . . . . . . . . . . . . 33

3.11 Adsorbed thickness . . . . . . . . . . . . . . . . . . 34

3.12 Initial condition limits $\ldots \ldots \ldots$

3.12 .1 Initial condition minimum . . . . . . . . . . . . . 35

3.12 .2 Initial condition maximum . . . . . . . . . . . . . . 36

4 Numerical Method of Solution $\quad 37$

4.1 The differential equation . . . . . . . . . . . . . . . . . 38

4.2 Algorithm outline . . . . . . . . . . . . . . . . . 38

4.3 Required inputs . . . . . . . . . . . . . . . . . 41

4.4 Initial conditions $\ldots \ldots \ldots \ldots \ldots$. . . . . . . . . . . 41

4.5 Applied perturbations $\ldots \ldots \ldots \ldots$. . . . . . . . . . . 42

4.5 .1 Perturbation limits . . . . . . . . . . . . . . . . 43

4.6 Parametric study . . . . . . . . . . . . . . . . . . . . . 44

4.7 The Hamaker constant . . . . . . . . . . . . . . . . . . . . . 44

4.8 Adsorbed thickness . . . . . . . . . . . . . . . . . . 45

4.9 Integral evaluation $\ldots \ldots \ldots \ldots \ldots \ldots \ldots$

4.9 .1 Thin film thickness $\ldots \ldots \ldots \ldots \ldots$

4.9 .2 Interfacial temperature $\ldots \ldots \ldots \ldots \ldots$

4.9 .3 Integral total $\ldots \ldots \ldots \ldots \ldots \ldots \ldots$

4.10 Runge-Kutta Solution _ . . . . . . . . . . . . . . . . 48

4.10 .1 Interface temperature $\ldots \ldots \ldots \ldots \ldots$

4.10 .2 Film thickness . . . . . . . . . . . . . . . . . . 51

4.11 Convergence criteria $\ldots \ldots \ldots \ldots \ldots \ldots$ 
4.11 .1 Interface temperature $\ldots \ldots \ldots \ldots \ldots$

4.11 .2 Film thickness . . . . . . . . . . . . . . . . . 52

4.12 Constant curvature . . . . . . . . . . . . . . . . . 52

4.13 Far field curvature . . . . . . . . . . . . . . 54

4.14 Complete model . . . . . . . . . . . . . . . . . . . . . . . 54

4.15 Sources of error . . . . . . . . . . . . . . . 56

5 Numerical Results $\quad 57$

5.1 Basic results . . . . . . . . . . . . . . . . . . . 59

5.2 Uniqueness study $\ldots \ldots \ldots \ldots \ldots \ldots \ldots$

5.3 Parametric study . . . . . . . . . . . . . . . . 75

5.3 .1 Thickness perturbation $\ldots \ldots \ldots \ldots \ldots \ldots$

5.3 .2 Slope perturbation $\ldots \ldots \ldots \ldots \ldots$

5.3 .3 Curvature perturbation . . . . . . . . . . . . 91

5.4 Marangoni effects . . . . . . . . . . . . . . . . . 96

5.5 Wall slip . . . . . . . . . . . . . . . . . . 103

5.6 Different fluids . . . . . . . . . . . . . . . . . 108

5.7 Different channel widths . . . . . . . . . . . . . . . . . 113

5.8 Different superheats . . . . . . . . . . . . . . . . . . . . . . . 119

6 Conclusions and Recommendations $\quad 124$

6.1 Conclusions . . . . . . . . . . . . . . . . . . . . . . 124

6.2 Recommendations . . . . . . . . . . . . . . . . . 128

$\begin{array}{ll}\text { List of References } & 130\end{array}$

$\begin{array}{ll}\text { Appendix A Derivation of Thin Film Model } & 134\end{array}$

Appendix B Determining the Adsorbed Film Thickness 150 
Appendix C Second Derivative Limits

Appendix D Reduction of Order 


\section{List of Tables}

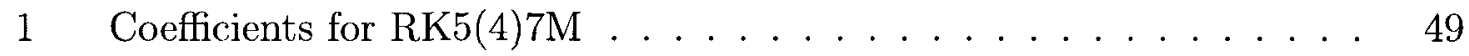

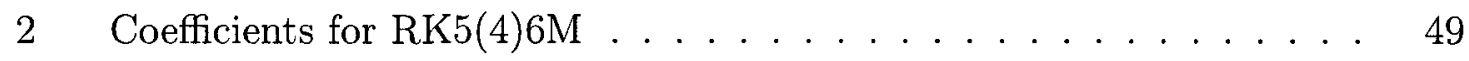

3 Available results for graphical plotting . . . . . . . . . . 55

$4 \quad$ Numerical model input values . . . . . . . . . . . . . . . . . . . 59

$5 \quad$ Perturbations for uniqueness test $\ldots \ldots \ldots \ldots$

6 Marangoni model input values . . . . . . . . . . . . . . . . . 96

$7 \quad$ Marangoni model input values . . . . . . . . . . . . . . . . . . 99

8 Slip length initial conditions . . . . . . . . . . . . . . . . . . 103

9 Pentane and octane initial conditions . . . . . . . . . . . . . 108

10 Channel width initial conditions . . . . . . . . . . . . . . 113

11 Initial conditions for varied superheats . . . . . . . . . . . . . 119 


\section{List of Figures}

1 Meniscus formed in a channel . . . . . . . . . . . . 3

2 Disjoining pressure via thin film interaction . . . . . . . . . 11

3 Thin film portion of a meniscus . . . . . . . . . . . . . 24

4 Main algorithm flowchart . . . . . . . . . . . . . . . 39

5 Runge-Kutta algorithm flowchart . . . . . . . . . . . . 40

6 Thin film thickness . . . . . . . . . . . . . . . 60

$7 \quad$ Local curvature . . . . . . . . . . . . . . . . . . . . 61

8 Evaporative $\operatorname{mass}$ flux $\ldots \ldots \ldots \ldots \ldots$

$9 \quad$ Local interface temperature . . . . . . . . . . . . . . 63

10 Local surface tension . . . . . . . . . . . . . . . . . . . . 64

11 Capillary pressure profile . . . . . . . . . . . . 65

12 Disjoining pressure profile $\ldots \ldots \ldots \ldots 6$

13 Liquid pressure profile $\ldots \ldots \ldots \ldots \ldots$

14 Composite pressure profiles $\ldots \ldots \ldots \ldots 6$

15 Liquid pressure gradient $\ldots \ldots \ldots$. . . . . . . . . . . 69

16 Thin film thickness profiles . . . . . . . . . . . . . . 71

17 Local curvature profiles . . . . . . . . . . . . . . . . . 72

18 Evaporative mass flux profiles . . . . . . . . . . . . . 73

19 Relationship between $\epsilon_{1}$ and $\delta^{\prime} \ldots \ldots \ldots \ldots \ldots \ldots$

20 Curvature variance with $\epsilon_{1} \ldots \ldots \ldots \ldots \ldots$ 
21 Thin film thickness variance with $\epsilon_{1} \ldots \ldots \ldots \ldots 78$

22 Curvature variance with $\epsilon_{1} \ldots \ldots \ldots \ldots \ldots$

23 Mass flux variance with $\epsilon_{1} \ldots \ldots \ldots$. . . . . . . . 80

24 Interface temperature variance with $\epsilon_{1} \ldots \ldots \ldots$

25 Capillary pressure variance with $\epsilon_{1} \ldots \ldots \ldots \ldots$. . . . . . . 82

26 Disjoining pressure variance with $\epsilon_{1} \ldots \ldots \ldots \ldots$

27 Liquid pressure variance with $\epsilon_{1} \ldots \ldots \ldots \ldots$. . . . . . 84

28 Liquid pressure gradient variance with $\epsilon_{1} \ldots \ldots \ldots$. . . . . 85

29 Curvature variance with $\epsilon_{2} \ldots \ldots \ldots \ldots$. . . . . . . . 86

30 Film thickness variance with $\epsilon_{2} \ldots \ldots \ldots \ldots$. . . . . . . 87

31 Curvature variance with $\epsilon_{2} \ldots \ldots \ldots \ldots \ldots$

32 Evaporative mass flux variance with $\epsilon_{2} \ldots \ldots \ldots$. . . . . . 89

33 Interface temperature variance with $\epsilon_{2} \ldots \ldots \ldots$

34 Thin film thickness variance with $t_{3} \ldots \ldots \ldots$. . . . . . . 92

35 Curvature variance with $\epsilon_{3} \ldots \ldots \ldots \ldots$. . . . . . . . 93

36 Evaporative mass flux variance with $\epsilon_{3} \ldots \ldots \ldots$. . . . . . 94

37 Interface temperature variance with $\epsilon_{3} \ldots \ldots \ldots \ldots$

38 Marangoni effects on thin film thickness . . . . . . . . . . 97

39 Marangoni effects on far field curvature . . . . . . . . . . . . . . 97

40 Marangoni effects on evaporative mass flux . . . . . . . . . . 98

41 Marangoni effects on interface temperature . . . . . . . . . . . . 98

42 Marangoni effects on thin film thickness . . . . . . . . . . 100

43 Marangoni effects on far field curvature . . . . . . . . . . . 100

44 Marangoni effects on evaporative mass flux . . . . . . . . . . . 101

45 Marangoni effects on interface temperature . . . . . . . . . . . . 101

46 Wall slip effects on thin film thickness . . . . . . . . . . . . . . 104

47 Wall slip effects on the curvature . . . . . . . . . . 105 
48 Wall slip effects on the evaporative mass flux . . . . . . . . . 106

49 Wall slip effects on the interfacial temperature profile . . . . . . . 107

50 Pentane and octane thin film thickness . . . . . . . . . . . . . 109

51 Pentane and octane curvature profiles . . . . . . . . . . . . 110

52 Pentane and octane evaporative mass flux profiles . . . . . . . . . 111

53 Pentane and octane interface temperature profiles . . . . . . . . . 112

54 Channel radius effects on thin film thickness . . . . . . . . . . . . 114

55 Channel radius effects on local curvature . . . . . . . . . . . 115

56 Channel radius effects on evaporative mass flux . . . . . . . . . 117

57 Channel radius effects on interface temperature . . . . . . . . . . 118

58 Superheat effects on thin film thickness . . . . . . . . . . . . 120

59 Superheat effects on curvature . . . . . . . . . . . . . . . 121

60 Superheat effects on evaporative mass flux . . . . . . . . . . . . 122

61 Superheat effects on interface temperature . . . . . . . . . . 123

62 Thin film portion of a meniscus . . . . . . . . . . . 134 


\section{Nomenclature}

\section{Latin}

\begin{tabular}{|c|c|c|}
\hline Symbol & Description & Units \\
\hline$A$ & Dispersion constant & {$[J]$} \\
\hline$A_{H}$ & Hamaker constant & {$[J]$} \\
\hline$\hat{A}$ & Area & {$\left[m^{2}\right]$} \\
\hline$a$ & Surface tension coefficient & {$\left[\frac{N}{m}\right]$} \\
\hline$b$ & Surface tension temperature coefficient & {$\left[\frac{N}{m K}\right]$} \\
\hline$C$ & Accommodation coefficient & {$[-]$} \\
\hline$C_{4}$ & Slip length relation & {$\left[m^{3}\right]$} \\
\hline$C_{5}$ & Slip length relation & {$\left[m^{2}\right]$} \\
\hline$d$ & Change of variable & {$[-]$} \\
\hline$G$ & Gibbs free energy & {$[J]$} \\
\hline$g_{x}$ & Gravitational acceleration & {$\left[\frac{m}{s^{2}}\right]$} \\
\hline$H$ & Channel height & {$[m]$} \\
\hline
\end{tabular}




\begin{tabular}{|c|c|c|}
\hline$h_{f g}$ & Latent heat of vapourization & {$\left[\frac{\mathrm{J}}{k g}\right]$} \\
\hline$h_{l v}^{c l}$ & Clapeyron evaporative mass flux coefficient & {$\left[\frac{k g}{m^{2} s K}\right]$} \\
\hline$h_{l v}^{k l}$ & Kelvin evaporative mass flux coefficient & {$\left[\frac{s}{m}\right]$} \\
\hline$i$ & Position counter along the thin film length & {$[-]$} \\
\hline$k_{B}$ & Stefan Boltzmann constant & {$\left[\frac{J}{s m^{2} K^{4}}\right]$} \\
\hline$k_{l}$ & Liquid thermal conductivity & {$\left[\frac{W}{m K}\right]$} \\
\hline$M$ & Molecular weight & {$\left[\frac{\mathrm{kg}}{\mathrm{kmol}}\right]$} \\
\hline$\dot{m}$ & Mass flow rate & {$\left[\frac{\mathrm{kg}}{\mathrm{s}}\right]$} \\
\hline$\dot{m}_{\text {evap }}^{\prime \prime}$ & Evaporative mass flux & {$\left[\frac{\mathrm{kg}}{\mathrm{m}^{2} s}\right]$} \\
\hline$P$ & Pressure & {$[P a]$} \\
\hline$P_{c}$ & Capillary pressure & {$[P a]$} \\
\hline$P_{d}$ & Disjoining pressure & {$[P a]$} \\
\hline$P_{l}$ & Liquid pressure & {$[P a]$} \\
\hline$P_{v}$ & Vapour pressure & {$[P a]$} \\
\hline$\dot{q}$ & Heat generation & {$\left[\frac{W}{m^{3}}\right]$} \\
\hline$R$ & Universal gas constant & {$\left[\frac{J}{m o l K}\right]$} \\
\hline$r$ & Channel radius & {$[m]$} \\
\hline$t$ & Time & {$[s]$} \\
\hline$T$ & Temperature & {$[K]$} \\
\hline
\end{tabular}




$\begin{array}{lll}T_{l v} & \text { Liquid vapour temperature } & {[K]} \\ T_{v} & \text { Vapour temperature } & {[K]} \\ T_{w} & \text { Wall temperature } & {[K]} \\ u & \text { Thin film velocity } & {\left[\frac{\mathrm{m}}{\mathrm{s}}\right]} \\ u_{s} & \text { Thin film velocity at the wall } & {\left[\frac{\mathrm{m}}{\mathrm{s}}\right]} \\ V_{l} & \text { Molar volume } & {\left[\frac{\mathrm{m}^{3}}{\mathrm{~mol}}\right]} \\ x & \text { Thin film length } & {[\mathrm{m}]}\end{array}$

\section{Greek Characters}

Symbol Description Units

$\alpha \quad$ Local curvature term

$\beta \quad$ Thin film wall slip length

$[m]$

$\delta \quad$ Thin film thickness $[m]$

$\delta_{0} \quad$ Adsorbed film thickness $\quad[m]$

$\epsilon_{1}$ Perturbation applied to $\delta_{0} \quad[m]$

$\epsilon_{2}$ Perturbation applied to $\delta^{\prime}$

$\epsilon_{3} \quad$ Perturbation applied to $\delta^{\prime \prime} \quad\left[\frac{1}{m}\right]$

$\epsilon_{4} \quad$ Perturbation applied to $T_{l v_{0}} \quad[K]$

$\eta \quad$ Kelvin-Clapeyron evaporative mass flux coefficient $\quad\left[\frac{\mathrm{mol}}{\mathrm{sm} m^{2}} K^{3 / 2}\right]$ 
Curvature gradient

$\left[\frac{1}{m^{2}}\right]$

$\lambda$ Collection of terms in ODE

$\mu$

Dynamic viscosity

$\left[\frac{N s}{m^{2}}\right]$

$\nu$

Kinematic viscosity

$\left[\frac{m^{2}}{s}\right]$

$\rho$

Density

$\left[\frac{\mathrm{kg}}{\mathrm{m}^{3}}\right]$

$\sigma$

Surface tension

$\left[\frac{N}{m}\right]$

$\tau$

Liquid vapour interfacial shear stress

$\left[\frac{N}{m^{2}}\right]$

$\chi$

Collection of terms in ODE

$\left[W m K^{3 / 2}\right]$

$\psi$

Collection of terms in ODE

$\left[\frac{1}{m K}\right]$

$\omega \quad$ Collection of terms in ODE

$\left[\frac{W K^{3 / 2}}{m}\right]$ 


\section{Subscripts}

\begin{tabular}{lll} 
Symbol & Description & Units \\
\hline guess & Guess value used in Runge-Kutta solver & {$[-]$} \\
in & Flow into the thin film & {$[-]$} \\
$l v$ & Liquid vapour interface & {$[-]$} \\
out & Flow out of the thin film & {$[-]$} \\
solved & Solved value used in Runge-Kutta solver & {$[-]$}
\end{tabular}

\section{Superscripts}

Symbol Description Units

Derivative with respect to $\mathrm{x} \quad[-]$ 


\section{Chapter 1}

\section{Introduction}

The unrelenting progression of science has taken humanity from the Stone Age through to the modern era of space flight. The greatest accomplishments to which we can lay claim stem from our inquisitive nature. The questions have progressed from the early days of Archimedes through to the middle ages of da Vinci, to what may now be considered the modern era of science. Recent strides in our understanding have come from people such as Newton, Einstein and Hawking. Theories of natural physics have provided the foundation, for our understanding of a range of phenomena from the galaxy to the behaviour of sub atomic particles.

With the solid foundations laid by previous generations of scientists and engineers alike, the continued study of the world in which we live, can be further explored. The advent of modern computing has allowed today's researchers to shed light on aspects of science which are of an interdisciplinary nature. It is this crossover of disciplines that holds great promise for the advancement of science and society. 
The philosophical foundations of modern science have allowed the steady progression of science and technology, such that we can study newly found aspects of engineering. The knowledge base held by engineers is continuously evolving as the understanding of the processes is better understood. This is most certainly true in the field of thin film heat transfer research.

The recent works of researchers have expanded the application of thin films to high performance phase change heat transfer devices [1]. These applications include spray cooling, heat pipes, capillary pumped loops and grooved evaporators [1-3]. The applications for such devices and methods have been reserved for heat flux problems, such as the cooling of central processing units, thermal distribution in satellite structures and the quenching of metals [4]. All of the aforementioned devices rely, in principle, upon the thermal properties of the evaporating thin film region of a meniscus.

\subsection{The thin film region}

As thin films play an important role in heat transfer, the definition of a thin film region needs to be specified explicitly. From the stand point of heat transfer and engineering, the thin film can be identified as a region of a liquid meniscus which approaches the wall and this thin film region forms the bridge between the adsorbed film and the bulk meniscus as shown in Figure 1. 


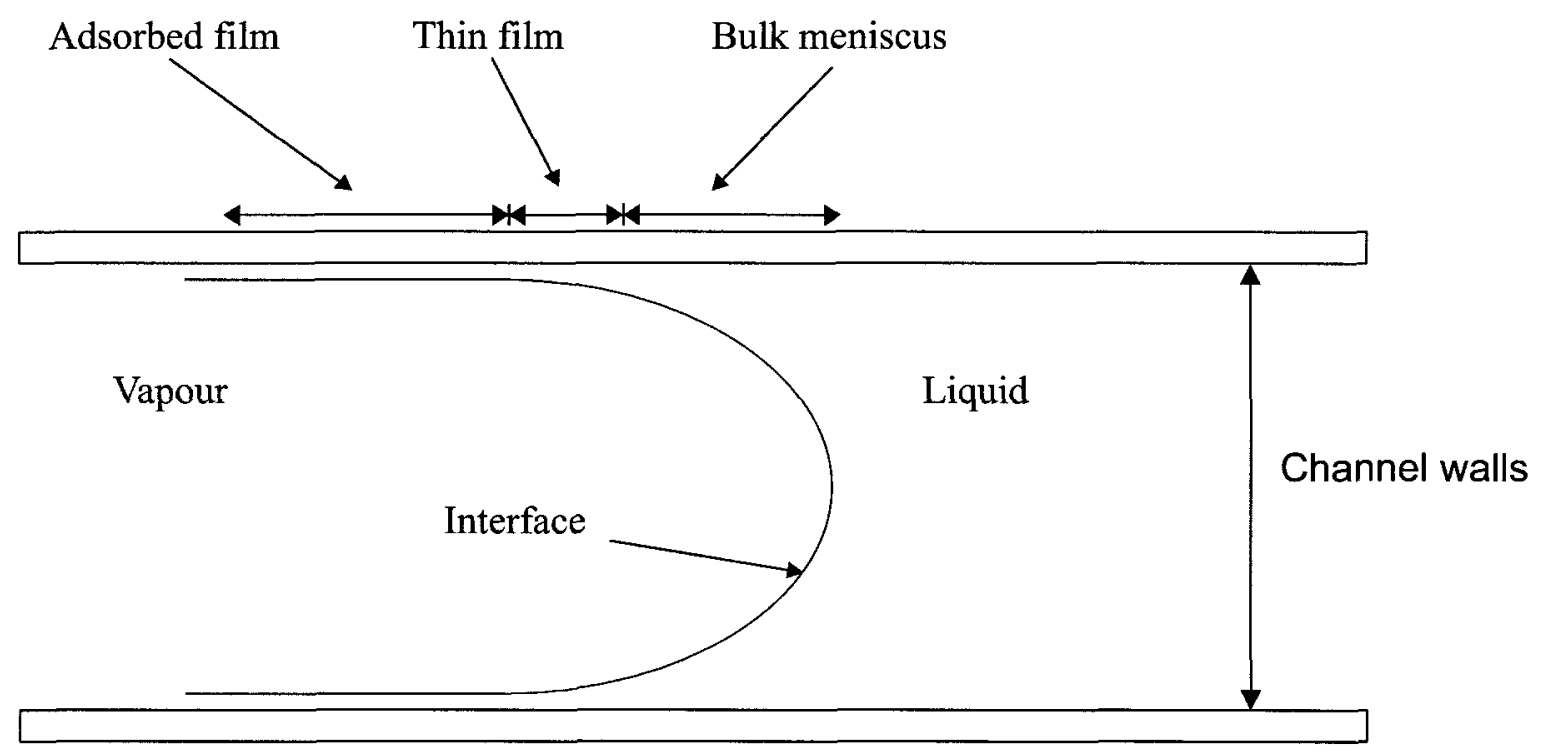

Figure 1: Meniscus formed in a channel

As illustrated, a meniscus and associated thin film can be formed by the close proximity of two walls. Other methods include the use of porous media such as a mesh structure, sintered material and grooved surfaces [5]. For the purpose of this thesis, the meniscus will be formed by the close proximity of two solid plates, such as that shown in Figure 1, that do not dissolve in the presence of the liquid within the channel.

The study of thin films and their associated thermal properties have been conducted both theoretically and experimentally. Of the theoretical methods, two approaches have been used. They include the application of continuum mechanics and molecular simulation. The continuum mechanics approach consists of two branches, the first requires the presence of an apparent contact angle at the start of the thin film region. The second involves the application of a set of perturbed initial conditions applied to an ordinary differential equation via perturbation theory. The second approach to understanding the thin film region is the application of molecular dynamics.

Both approaches to solving the thin film region have their merits and limitations. 
For the case of the apparent contact angle, this value cannot be directly obtained, and as stated by Wayner Jr. [6], vanishes on the molecular scale as you approach the adsorbed film region. For both methods of contact angle and continuum mechanics, they apply equations at a macro scale through to the adsorbed region. It is in this adsorbed region that the works of Derjaguin and Hammaker come to light, as the dominant forces shift toward the application of van der Waal's forces [7]. Despite this shift in force dominance, both methods account for this shift in force with the application of disjoining pressure.

\subsection{Research motivation}

The engineering applications of phase change heat transfer devices such as heat pipes, have lead to the refinement of thin film understanding. The limitations of such devices can be determined experimentally with the use of a well structured procedure. This method of investigation is slow and labour-intensive. As such, researchers continue looking for a means by which to theoretically determine the heat transport capabilities of such devices. As previous works have suggested, the bulk of the evaporation is expected to occur in the thin film region of a meniscus.

Thus the primary objective of this research is to develop a mathematical model to accurately describe an evaporating thin film. This model is meant to characterize and quantify the evaporative characteristics of the thin film region. The goals of this model are to include the effects of a liquid-vapour surface temperature gradient, slip at the liquid-solid interface, changes in applied superheat, different types of fluids and variation in channel width. Each of the aforementioned can be associated with change in the heat transport capability of the thin film, and are investigated individually. Lastly, the thin film length as found in this model will to be used in the study of thin film stability. 


\subsection{Thesis outline}

This thesis presents the development and solution of a mathematical model formulated to describe an evaporating thin film. This model is then used to simulate and analyze different aspects of its behaviour and range of applications. The structure of the thesis is organized as follows:

\section{Chapter 1: Introduction}

Provides an introduction to the progression of science and engineering, with a description of where a thin film occurs, and a statement of the problem to be investigated.

\section{Chapter 2: Literature Review}

Presents a thorough review of the theory and principles, that govern the evaporation process in thin films.

\section{Chapter 3: Mathematical Model}

Provides a concise formulation of the mathematical model and all associated boundary conditions.

\section{Chapter 4: Numerical Method of Solving}

Presents a description of how the governing differential equations are solved, as well as the details in determining initial conditions and the imposition of limits.

\section{Chapter 5: Numerical Results}

Provides a case by case analysis, with important results highlighted and discussed as they relate to published and experimental works.

\section{Chapter 6: Conclusions and Recommendations}

Presents conclusions based on results for each of the cases analyzed, and gives recommendations for future work. 


\section{Chapter 2}

\section{Literature Review}

The properties of bulk liquid phase change do not directly correlate with that of thin

films. As such, the observed properties of thin films deviate from traditional theories. Thus, new theories and methods of analysis have been presented by researchers and they continue to progress. The details of previous thin film theories are presented herein.

\subsection{The meniscus}

The interface formed between a liquid and a vapour for a large reservoir, such as a bucket full of water, is considered planar for the majority of the surface area. However, where the edges of the interface make contact with the solid substrate, there exists a small curved region. When the scale of the fluid reservoir is reduced, the interface increases in curvature to a point such that the radius can be observed and measured. This allows for the further breakdown of the meniscus into three regions: the adsorbed film, thin film and bulk meniscus.

The concavity of the meniscus is determined by the cohesive interaction of the liquid with that of the solid substrate. For a convex meniscus, the liquid molecules have a greater affinity for each other, than that of the solid substrate. Conversely, 
for the concave meniscus, the liquid molecules have a greater affinity for the solid substrate than the bulk liquid. This phenomena is described as wetting, and is defined for a 'solid-liquid set' based on a measured contact angle [8]. For the purposes of this research, only the concave meniscus is considered, as this form is the dominant type found in heat transfer devices.

\subsubsection{Adsorbed region}

The adsorbed region of the meniscus is characterized by the dominance of van der Waal's forces as a result of the interactions between liquid and solid substrates [9]. This adherence to the solid substrate is a form of pressure, first conceptualized by Derjaguin et al. [7], as disjoining pressure. As a result of the adhesive forces in this region, the superheated liquid molecules are restrained from evaporation $[9,10]$. Thus, the adsorbed layer has been described in recent works as a uniform film of even thickness extending from the thin film region for an unspecified length $[2,3,6,10-12]$.

With these descriptions for the adsorbed region, researchers have made efforts to quantify some aspects of the thin film from their inferences and assumptions. Firstly, from the uniform thickness of the adsorbed film, it has been inferred that the curvature is zero and constant [10]. This curvature condition allows for the determination of initial conditions regarding the thickness and associated derivatives. The next inference stems from the adsorbed layers inability to undergo evaporation. As the adsorbed layer is thin, it has been suggested that heat would be transported through the film via conduction, and that it would be of the same temperature as the solid substrate $[5,9,11]$.

As for the determination of the adsorbed film thickness, many researchers have calculated its thickness for a given superheat [5,12]. Even so, some researchers assigned a constant thickness value that is of the expected order of magnitude $[5,13,14]$. 


\subsubsection{Thin film region}

As the region spanning between the adsorbed film and the bulk meniscus, the thin film region is expected to share traits of both. Thus, the contributions of disjoining pressure and capillary pressure are of equal importance over this region [9].

Starting with the joining of the thin film region to the adsorbed region, the curvature at the joint is expected to be near zero $[5,9]$. Given that the thickness of adsorbed layers are of a nanometer scale, most researchers employed continuum mechanics $[2,5,6,9,11-22]$, with only one research team considering the application of molecular dynamics closest to the adsorbed region [23]. Given that the thin film region nearest the adsorbed layer is of comparable thickness, the thermal conductive losses should be small [5]. The increase in thickness is sufficient so as to permit evaporation, resulting from the decay in disjoining pressure.

As the thin film transitions toward the bulk region, it is characterized by the transition of dominant pressure forces from that of the disjoining to capillary $[11,15]$. This transition is driven by the change in thickness and the subsequent change in curvature. As the thin film region grows in thickness the disjoining pressure decreases in strength. All the while, the capillary pressure is increasing as the film thickness transitions from the constant, near zero curvature in the adsorbed region to a different curvature in the bulk region.

\subsubsection{Bulk meniscus region}

Of the three zones composing the meniscus region, the bulk meniscus region makes up the majority of the interface and is of a macro scale. Within the bulk meniscus, the capillary pressure is dominant, and the disjoining pressure is near zero $[5,9,15]$. Previous studies into phase change heat transfer suggest that this region has a small contribution to the evaporative mass flux $[5,17,24]$. 
The transition from the bulk meniscus region to that of the thin film has been of particular interest. The contributing conditions proposed include the achievement of a constant curvature value or the assignment of a capillary pressure cutoff value $[3,5$, 9]. Of these selection criteria, the dominant choice has been the constant curvature condition. As such, many researchers have assigned a $1 / r$ value for the constant curvature so as to keep with the description of the Young-Laplace equation [10].

\subsection{Thin film applications}

The potential heat transport capability of evaporating thin films are predominantly found in devices such as heat pipes, loop heat pipes, capillary pumped loops, grooved evaporators, nucleate boiling and coatings $[5,9,15,16,25]$. Such devices have been employed to maintain the operating temperature of central processing units through to spacecraft payloads. With the progression of computing technologies toward smaller systems, the thermal management of high power density devices is of a growing con-

cern [26]. For the increasing need to dissipate high heat fluxes quickly and efficiently, phase change heat transport devices which employ thin films, are of great importance.

\subsection{Model development}

With the applications and refinement of thin films, in use today, many researchers have begun working on ways by which to better represent the thin film. Of particular importance has been to quantify characteristics of the thin film such as its size and heat transport capability. Such investigations have lead to the development of thin film models for which, researchers can change and modify aspects such as geometry, types of fluids and applied heat loads. Thus, the development of thin film models can be used to help engineers in determining the size and suitability of phase change 
heat transfer devices. The models developed to represent thin films have progressed from the explanation of shape and interfacial pressure jump to the prediction of the phase change process itself.

\subsubsection{Young-Laplace}

The early works of Young and Laplace, lead to the development of the Young-Laplace equation of capillarity, so as to describe the properties of a curved liquid-vapour interface. For an interface to be curved, there must be a pressure difference between the two phases [27]. Thus, the Young-Laplace equation relates the pressure difference between the two immiscible phases to the curvature [27]. With the assumptions of thermodynamic equilibrium, steady state and no gravity, the Young-Laplace equation can be written as [27]:

$$
\Delta P=\sigma_{l v}\left(\frac{1}{r_{1}}+\frac{1}{r_{2}}\right)
$$

Where the equation is written for an interface composed of two principle radii $r_{1}$ and $r_{2}$. For a channel composed of two parallel, semi-infinite plates, the second principle radius can be assumed to approach infinity. This leads to a form of the Young-Laplace equation for a channel of height $2 r$ given by:

$$
\Delta P=\frac{\sigma_{l v}}{r}
$$

Where the $1 / r$ term represents the local curvature $(\kappa)$ of the interface. In addition, the interfacial pressure jump described by $\Delta P$ is from here forward referred to as capillary pressure as:

$$
P_{c}=\sigma_{l v} \kappa
$$




\subsubsection{Disjoining pressure}

The concept of disjoining pressure was first proposed by Boris Derjaguin in the mid nineteen thirties as a means of explaining solid-liquid thin film interactions. The study of colloids, foams, thin films, wetting and many other systems require the understanding of the Gibbs layer interactions [7]. The simplest description of disjoining pressure was given by Derjaguin et al. [7], as the interaction of two plates submerged in a homogeneous fluid. The interaction between the fluid and solid plates occurs at the molecular level as a result of van der Waal's interactions. This interaction results in the formation of a thin film encasing the plates. The interaction between the bulk liquid and the solid substrate alter the bulk fluid such that the thin film exhibits different properties than that of the bulk fluid [7]. Illustrating the presence of the thin film layers on the solid substrate Figure 2(a) and the interaction of the thin film layers in Figure 2(b) [7].

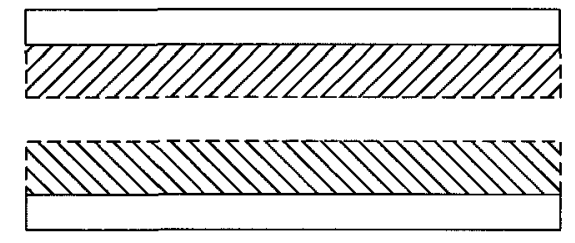

(a) Thin film layers

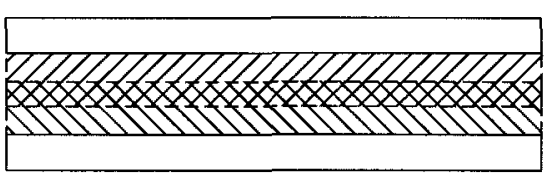

(b) Thin film interaction

Figure 2: Disjoining pressure via thin film interaction

This interaction of the thin film layers, generates a disjoining pressure. As such, Derjaguin defined disjoining pressure as the difference in pressure between that of the bulk fluid and the thin film fluid [7]. This was formulated using Gibbs free energy, as a function of the distance between the plates and the area normal to that of the plates. This is represented by Equation (4), for a constant area, temperature and pressure [27]: 


$$
P_{d}=-\frac{1}{\hat{A}}\left(\frac{\partial G}{\partial x}\right)_{\hat{A}, T, P}
$$

In combining the contributions of Hamaker, the disjoining pressure occurring within the thin film can be related to the thickness of the thin film. The Hamaker derivation defines a force per unit area for an infinite plate via Equation (5) [28]. This in essence, defines the relation for the disjoining pressure as a function of film thickness $(\delta)$ and the Hamaker constant $\left(A_{H}\right)$.

$$
P_{d}=\frac{A_{H}}{6 \pi} \frac{1}{\delta^{3}}
$$

This definition of the disjoining pressure is only applicable to non-polar fluids. To further simplify the relation for disjoining pressure, the dispersion constant is defined as:

$$
A=\frac{A_{H}}{6 \pi}
$$

Leading to the final form of the disjoining pressure as a function of film thickness and the dispersion constant.

$$
P_{d}=\frac{A}{\delta^{3}}
$$




\subsubsection{Hamaker constant}

The Hamaker constant is not an actual constant, but was originally defined as a function of the London-van der Waals constant and material bulk density [28]. The understanding of the Hamaker constant has since been broken down into three main components which include the Keesom, Debye and London interactions [27]. From these three principle molecular interactions, the Hamaker constant can be approximated using Equation (8) [10,27] as:

$$
A_{131}=\frac{3}{4} k_{B} T\left(\frac{\epsilon_{1}-\epsilon_{3}}{\epsilon_{1}+\epsilon_{3}}\right)^{2}+\frac{3 h \nu_{e}}{8 \sqrt{2}} \frac{\left(n_{1}^{2}-n_{3}^{2}\right)^{2}}{\left(n_{1}^{2}+n_{3}^{2}\right)^{3 / 2}}
$$

This form of the Hamaker constant is used for a symmetric case where the working fluid (3), is balanced on either side with a solid substrate (1). The geometric nature of the system for which Equation (8) is associated, requires a planar interface with no curvature. These are the conditions which make-up the subscript (131) of the Hamaker constant described by Equation (8). The value of the Hamaker constant is determined from the dielectric constants $\epsilon_{j}$, and refractive indices $n_{k}$, for the associated materials. This method of determining the Hamaker constant is an approximation and is valid provided the London dispersion interaction is greater than the sum of the Keesom and Debye interactions [27].

Of the Hamaker constant approximations, two other types have been proposed as the simple spectral method and the full spectral method [27]. The full spectral method has been investigated by French et al. [29], for the purposes of determining the Hamaker constant for ceramic systems. Their work into multi-layered systems has illustrated that the Hamaker constant is a function of each layer and their associated thickness. This approach to multi-layer systems or thickness dependence is not present in any of the works into evaporating thin films. Thus, it is possible to surmise that the disjoining pressure effects in the thin film region may have a more complex 
geometric relation. This relation is a result of the variance in thin film thickness with film length.

\subsubsection{Augmented Young-Laplace}

The Young-Laplace equation fails to account for the disjoining pressure affect present in thin films. This comes from the fact that disjoining pressure was not realized until the early twentieth century. Given the effect that disjoining pressure imposes on the shape and thermodynamic properties, Wayner Jr., proposed an augmented form of the Young-Laplace equation [13].

$$
P_{v}=P_{l}+P_{c}+P_{d}
$$

The augmented form of the Young-Laplace equation provides a representation of the force balance acting upon the evaporating thin film. With this relation the film profile can be defined based on the physical properties of the fluid and its flow behaviour. Conversely, the thin film profile can be measured and used as input data, so as to obtain the flow properties as was done by Panchamgam et al. [16].

\subsubsection{Evaporation model}

The interactions which occur across a curved interface such as that of an evaporating thin film have been analyzed using aspects of thermodynamics and kinetic theory [30]. An early model for evaporation was presented by Schrage, which related the interfacial conditions with the flux across an interface [13]. The formulation defined the interfacial mass flux $\left(\dot{m}^{\prime \prime}\right)$, as a function of the jump conditions at the interface for both pressure and temperature [30].

$$
\dot{m}_{\text {evap }}^{\prime \prime}=C_{1}\left(\frac{M}{2 \pi R T_{l v}}\right)^{1 / 2}\left(P_{l v}-P_{v}\right)
$$


The continued development of an evaporation model was conducted by Wayner et al. [31], for which they formulated a relation to explain the evaporative process in thin films. Thus, Wayner et al. [17] put forth the Kelvin-Clapeyron model, so as to relate the interfacial mass flux to that of the interfacial temperature jump (Clapeyron effect) and the interfacial pressure jump (Kelvin effect).

$$
\dot{m}_{e v a p}^{\prime \prime}=\frac{1}{h_{f g}}\left(h_{l v}^{c l}\left(T_{l v}-T_{v}\right)-h_{l v}^{k l}\left(P_{d}+P_{c}\right)\right)
$$

Given,

$$
\begin{gathered}
h_{l v}^{c l}=\eta\left(\frac{1}{T_{l v}}\right)^{3 / 2}\left(\frac{M h_{f g}}{T_{v}}\right) \\
h_{l v}^{k l}=\eta\left(\frac{1}{T_{l v}}\right)^{3 / 2} V_{l}
\end{gathered}
$$

This evaporative mass flux model has been extensively used to predict the variance and location of evaporation and condensation $[2,5,9,11-13,15-17,19,21,30]$.

\section{$2.4 \quad$ Numerical studies}

From the aforementioned models and developments, researchers have been conducting several numerical simulations. Of the research conducted, three different approaches have been employed. They include computational fluid dynamics, molecular dynamics and derived ordinary differential equations for continuum mechanics. The most prevalent form of simulation uses the equations of continuum mechanics to form a set of ordinary differential equations to represent evaporating thin film characteristics. 


\subsubsection{Types of numerical results}

The simulations conducted so far have employed an assortment of differential equations, with various associated boundary conditions. The objectives of each simulation appeared to be at the discretion of the researcher so as to find information specific to their needs.

\subsubsection{Methods of solution}

The differential equations have been solved in a variety of methods which include:

- Runge-Kutta

- Finite element method

- Adams method

Of these methods, the Runge-Kutta method has been used extensively to determine the thin film characteristics such as film length $[3,5,9,14,15,32]$. This method of solution for ordinary differential equations requires the input of initial conditions for all of the derivatives. As noted earlier, the derivatives associated with the film thickness should be zero at the transition point between the adsorbed region and the thin film region. However this set of initial conditions would result in a trivial solution. Thus, the Runge-Kutta method requires a set of perturbed initial conditions. The magnitudes of the perturbations were generally small, but arbitrary in value. Of note is the lack of relationship between the derivative perturbations with respect to each other, or the level of applied superheat $[5,12,14,15]$. In contrast to the applied perturbations to the derivatives, Park et al. [15], claim to have solved a third order differential equation using Runge-Kutta, and the initial conditions set to those of the adsorbed region. The means by which Park et al. [15], were able to avoid reaching a trivial solution were not provided. 
The method used by Panchamgam et al. [16], combined experimental data with that of a finite element method model for the solid substrate and a continuum fluid dynamics model. This joining of experimental data with that of a model allowed for an iterative search to find a solution for the thin film profile with respect to a thermally conductive solid substrate. This solution method is robust, yet required the input of experimentally measured values smoothed by numerical curve fitting so as to achieve a continuous function [16].

\subsubsection{Equation behaviour}

The governing differential equation is of either second, third or fourth order $[3,5$, $9,11,12,14-16,19,32]$. The fourth order governing equations are that of third order equations with the application of one more differentiation with respect to the thin film length. The increase in order applied to the governing equation is done so as to remove the integration term associated with the liquid pressure gradient and evaporative mass flux $[5,12]$. This differentiation removed the need to have knowledge of the thin film thickness and interface temperature at the current point in a Runge-Kutta solution scheme.

With the Runge-Kutta method of solving being dominant, the perturbation stability characteristics of the third and fourth order governing equations were partially investigated. The perturbation effects to third order models were discussed by Wee et al. [9] and Zhao et al. [3], so as to characterize the effects of initial condition perturbations on the thin film profile. The numerical model developed by Wee et al. [9], solved for the first derivative of thin film thickness, while holding all other initial conditions constant, suggesting a sensitivity to the first derivative. In addition, Zhao et al. [3], stated a strong influence from both the initial thickness and first derivative perturbations. For the fourth order governing equations, Wang et al. $[5,12]$, showed only a sensitivity to the second derivative perturbation. 


\subsubsection{Trends in numerical results}

The results of numerical simulations, such as completed in previous works $[3,5,9,11$, $12,14-16,19,32]$, have three predominant variances that include:

- Channel width

- Applied superheat

- Wall slip

The width of the channel or tube where the meniscus is formed, can be varied so as to determine the effects on the evaporative capability of the thin film. Thus, the variance in channel width was investigated to determine its effects on thin film profile, and evaporative properties [11]. The trend observed in numerical models suggests an increase in thin film length with increase in channel width [11]. Second, the applied superheat at the walls of the channel can be varied by the applied heat load. The change in applied superheat results in the decrease in thin film length with increasing superheat [3]. Lastly, is the effects of slip length and the solid-liquid interface has been observed to increase the length of the thin film region $[9,15]$. 


\subsection{Alternate models}

The models developed to describe the thin film have been extensively that of continuum fluid mechanics and solved for two dimensional problems. These models have been complimented by the development of two different types of models, the first uses computational fluid dynamics (CFD), and the second uses molecular dynamics. The use of CFD suites such as FLUENT have allowed for the expansion of the evaporating thin film from that of a two dimensional entity to that of three dimensional one. The modeling of evaporating thin films using CFD has been investigated for different types of wick structures for applications in heat pipes [33,34]. These CFD models illustrate the three dimensional nature of the flow in the bulk meniscus and how it relates to the formation of vortical structures and temperature distributions [22]. With these comprehensive three dimensional models, Ranjan et al. [33,34], have described the evaporative characteristics for different geometries and superheats. Lastly, the effects of temperature gradients in conjunction with gravitational effects on horizontal menisci, have been shown to affect the structure and size of Marangoni vortices [22].

The second type of model not in common use is that of molecular dynamics based simulations. The model as developed by Maroo et al. [23], describes the formation and operation of an evaporating meniscus. The simulation employed equations to describe atomic interaction between the two phases of the evaporating system [23]. The evaporative process as modeled on the atomic scale is used due to the scale of the adsorbed thin film, where molecular forces are expected to dominate. The results show the transient aspect of thin film evaporation, which extends to the evolution of disjoining pressure, capillary pressure and vapour pressure [23]. 


\subsection{Experimental observations}

The majority of the work in thin film research has been done with numerical techniques as discussed previously. This tendency to numerically simulate thin film evaporation is due to the fact that the thin film region is at a scale which is difficult to observe directly. Thus few experiments have been performed where direct measurements could be achieved. Of the experiments conducted, the observations and measurements are either optical or thermal. Despite the limiting measurement resolution, these experiments have been proven beneficial in confirming some of the fundamental assumptions used in numerical models.

However similar the experiment to numerical simulation, differences between current models and experiments exist. These differences may include but not limited to:

- transient effects

- temperature gradients

- radiation

- surface roughness

- fluid purity

- convection

Of the experiments conducted so far, the geometric aspects of the thin film have been experimentally investigated for cylindrical tubes and square corners. The works of $\mathrm{Qu}$ et al. [11] and Buffone et al. [24], have made geometric measurements of the meniscus shape for cylindrical tubes. Both investigations confirm a transient aspect of the thin film geometry based on applied power loading. The thin film is measured to decrease in contact angle as the applied heat load is increased $[11,24]$. 
The investigation of meniscus geometries formed in the corner of a cuvette were investigated by Panchamgam et al. [16,35], as a means of quantifying the geometric properties of the thin film. Once again the transient aspect of the thin film shows an increase in the applied power results in an apparent oscillation in thin film length as the thin film stabilizes to a steady state of evaporation [35]. Of the results obtained, Panchamgam et al. $[16,35]$, were able to successfully use the experimentally measured thickness and curvature values in a numerical model. This merging of theoretical and experimental data, suggests the continuum models in micro fluid flow may be valid [16]. Lastly, the use of a slip boundary condition between the liquid and the solid substrate was needed to explain the high heat fluxes observed [16].

Aside from optical measurements of the meniscus shape, some researchers have been able to make measurements of the solid substrate temperature profile. Temperature profiles such as those obtained by Höhmann et al. [8], show a significant wall temperature drop in the thin film region before rebounding to the temperature in the bulk meniscus region. This temperature drop at the wall was attributed to high evaporative rates in the thin film region, and the rebound in temperature was a result of convection in the bulk meniscus region [8]. This work was complimented by that of Sodtke et al. [1], in their efforts to measure the temperature profile of a single vapour bubble. Much the same as the results of Höhmann et al. [1], a wall temperature drop was found to occur in the thin film region, after which the temperature rebounds.

\subsection{Summary and objectives}

The types of devices used to facilitate phase change heat transfer are as wide and varied as their applications. As a result, the study of menisci and their phase change characteristics are of great interest to engineers and scientists. From the basic formation of a meniscus to the subsequent dissection into three main regions, the thin film 
portion has become the focus of much interest. To further understand the heat transport capabilities of the thin film region, numerical models and experimental setups have been used to assign quantitative values to the thin film region.

As such, numerical investigations of thin film characteristics and behaviour previously conducted $[3,5,9,11,12,14-16,19,32]$, have covered a variety of initial conditions, assumptions and desired results. The formulations and assumptions for thin film numerical model from previous works are each different and distinct. The differences stem from early attempts to understand the thin film region, resulting in the evolution from wetting contact angles to that of asymptotic fluid mechanics. These differences have lead to a wide scope of results and conclusions from which many inferences have been drawn.

The experimental study of thin films $[1,8,11,16,24,35]$, have been limited in scope and measurement resolution. The difficulties in experimental research have limited data collection to that of optical and thermal measurements on a sub-micrometer scale [16]. Despite the resolution short comings, the experimental results have provided a means of confirming basic assumptions used in numerical studies.

With the collected works of those previous, it is the objective of this work to formulate a coherent numerical model to represent an evaporating thin film. The mathematical model will then be solved numerically so as to obtain quantitative data. The data obtained from the numerical simulations will provide information regarding the shape, curvature, interface temperature, evaporative mass flux and any other data as required. The focus of the data collected, will be towards that of varied superheats, channel widths, wall slip, Marangoni effects and different fluids. Of the data collected a direct comparison will be conducted so as to confirm the trends in thin film variation with both numerical and experimental data. 


\section{Chapter 3}

\section{Mathematical Model}

For the purposes of this formulation, the evaporating thin film region is schematically represented by Figure 3. As stated previously, the thin film region forms the region between the bulk meniscus and the adsorbed region. Given the geometry of the thin film, a Cartesian coordinate system was chosen such the $x$ direction defines the thin film length, $y$ defines the thickness and $z$ defines the depth of the formed meniscus. The origin of the coordinate system is placed at the surface of the heated wall and the end of the adsorbed thin film. The position which describes the end of the evaporating thin film and the start of the bulk meniscus will be determined by a set of conditions solved by the model. A comprehensive version of the derivation is provided in Appendix A. 


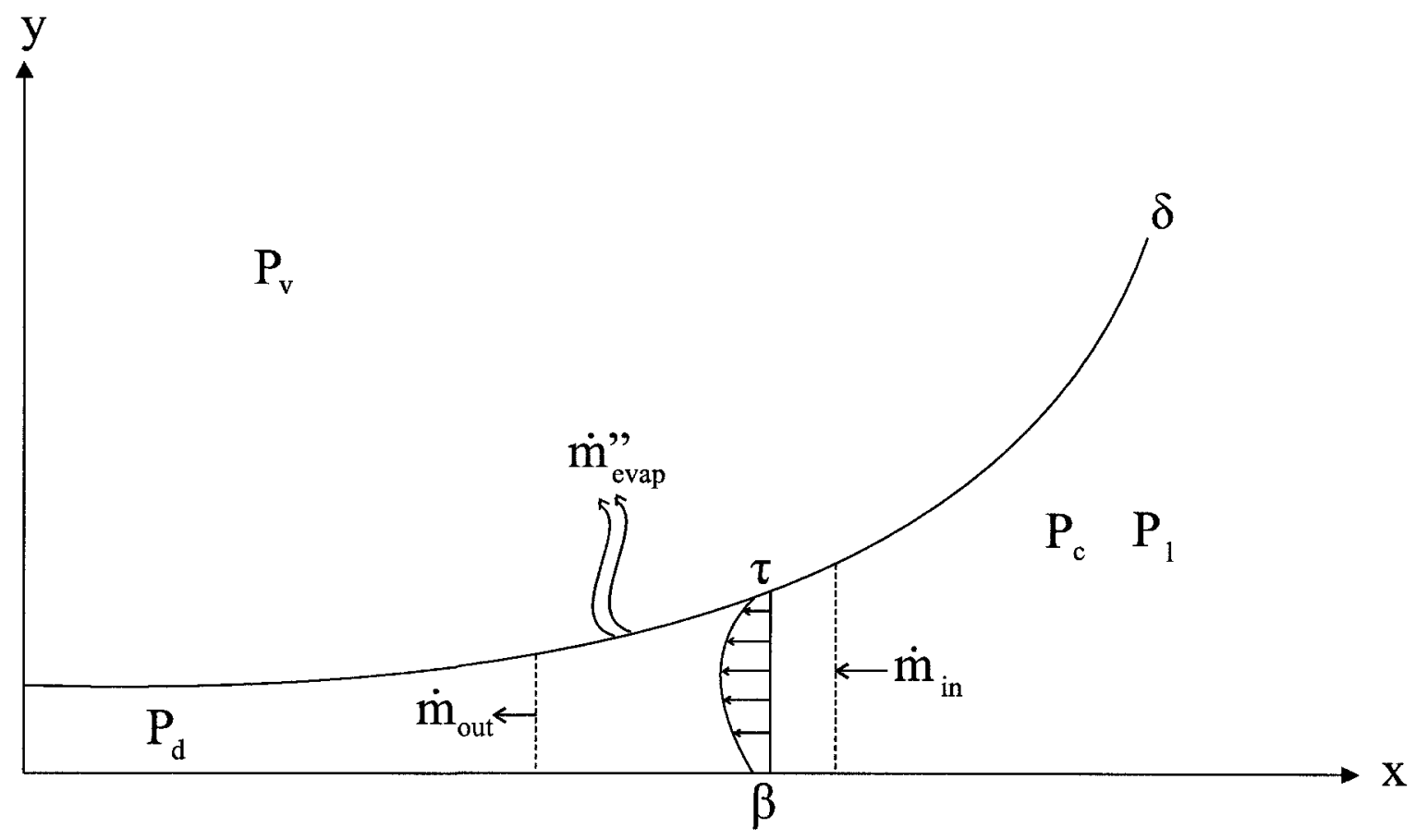

Figure 3: Thin film portion of a meniscus 


\subsection{Assumptions}

The governing equations for which the simulation is to be formed, require some knowledge of the system. Assumptions affecting the model as a whole include:

Purity

The working fluid of the system is assumed to be pure

Steady state

During evaporation, the system is assumed to be operating at steady state

\section{Vapour pressure}

The vapour pressure is assumed to be uniform and constant

\section{Wall substrate}

The walls are assumed to be of uniform temperature, with a smooth and planar surface and chemically neutral

\section{Meniscus depth}

The depth of the meniscus is assumed to be unity

\section{Liquid flow}

The liquid in the thin film does not flow in either the $y$ or $z$ directions

\section{Convection}

Convective effects are assumed negligible in both the vapour and liquid portions

\section{Radiation}

Radiative terms are assumed to be negligible

\section{Adsorbed region}

The intermolecular forces acting upon the adsorbed layer prohibit evaporation 


\subsection{Force balance}

To start the formulation of the mathematical model, the forces acting upon the thin film region are described by:

$$
P_{v}=P_{l}+P_{c}+P_{d}
$$

The pressures are defined as:

$$
\begin{gathered}
P_{c}=\sigma \kappa \\
P_{d}=\frac{A}{\delta^{3}} \\
P_{v}=\text { constant }
\end{gathered}
$$

Where the local curvature is described as:

$$
\kappa=\frac{\delta^{\prime \prime}}{\left(1+\left(\delta^{\prime}\right)^{2}\right)^{3 / 2}}
$$

The last remaining term of the force balance requires knowledge of the liquid pressure $P_{l}$, as a function of position. This can be obtained in the form of a differential, from the Navier-Stokes equations. Thus the relation for the liquid pressure gradient can be obtained by differentiating Equation (14) with respect to $x$. After differentiating and some manipulation the third order differential equation is obtained as:

$$
\delta^{\prime \prime \prime}=\frac{3 \delta^{\prime} \delta^{\prime 2}}{\alpha}+\frac{\alpha^{3 / 2}}{\sigma}\left(\frac{3 A}{\delta^{4}} \delta^{\prime}-\frac{d P_{l}}{d x}\right)-\frac{b}{\sigma}\left(\frac{d T_{l v}}{d x}\right) \delta^{\prime \prime}
$$


As desired, the thin film profile is now described in the form of a third order, ordinary differential equation (ODE). This form of the ODE requires knowledge of the liquid pressure gradient and the interfacial temperature gradient. Now, with the ODE in its current form, the liquid pressure gradient can be obtained directly from a solution to the Navier-Stokes equation.

\subsection{Navier-Stokes equations}

Given the coordinate system, the set of three Navier-Stokes equations can be used. However, flow in the directions of $y$ and $z$ are assumed to be zero, thus reducing the three principle equations to one in the $x$ direction [36].

$$
\rho\left(\frac{d u}{d t}+u \frac{d u}{d x}+v \frac{d u}{d y}+w \frac{d u}{d z}\right)=-\frac{d P_{l}}{d x}+\mu\left(\frac{d^{2} u}{d x^{2}}+\frac{d^{2} u}{d y^{2}}+\frac{d^{2} u}{d z^{2}}\right)+\rho g_{x}
$$

\subsubsection{Assumptions}

The flow is assumed to be uniform in the $x$ direction, with no velocity in the $y$ or $z$ directions, and the effects of gravity are neglected. Applying these assumptions to Equation (20) we get,

$$
\frac{d P_{l}}{d x}=\mu \frac{d^{2} u}{d y^{2}}
$$




\subsubsection{Boundary conditions}

The boundary conditions as they apply to Equation (21) are applied at the liquidvapour interface and at the solid-liquid wall.

At the wall, $y=0$, a slip velocity is permitted via:

$$
u=u_{s}=-\beta \frac{d u}{d y}
$$

At the liquid-vapour interface, $y=\delta$, a shear condition is permitted via:

$$
\tau=\sigma^{\prime}=\mu \frac{d u}{d y}
$$

Thus, applying boundary conditions (22) and (23) to Equation (21) and integrating from 0 to $\delta$ we obtain the liquid velocity profile.

$$
u=\frac{1}{\mu} \frac{d P_{l}}{d x}\left(\frac{y^{2}}{2}-\delta(y-\beta)\right)+\frac{b}{\mu} \frac{d T_{l v}}{d x}(y-\beta)
$$

\subsection{Continuity equation}

Considering the liquid velocity profile inside the thin film region, it is now possible to apply the continuity equation. This will provide the mass flow rate as a function of the liquid pressure gradient and liquid-vapour interfacial temperature gradient. Thus the cross-sectional area required by the continuity equation is taken to be unity in the $z$ direction and $d y$ as the film thickness.

$$
\dot{m}=\rho A u
$$


Thus the mass flow rate in the thin film region is a function of the velocity profile defined by Equation (24). Integrating Equation (25) from 0 to $\delta$ then provides the mass flow rate as a function of the interfacial temperature gradient and the liquid pressure gradient.

$$
\dot{m}=\frac{1}{\nu} \frac{d P_{l}}{d x}\left[-\frac{\delta^{3}}{3}+\beta \delta^{2}\right]+\frac{b}{\nu} \frac{d T_{l v}}{d x}\left[\frac{\delta^{2}}{2}-\beta \delta\right]
$$

\subsection{Energy equation}

As heat is applied through the channel walls, it is conducted through the thin film region. This conduction is considered in the application of the energy equation [37].

$$
\frac{\partial}{\partial x}\left(k_{l} \frac{\partial T}{\partial x}\right)+\frac{\partial}{\partial y}\left(k_{l} \frac{\partial T}{\partial y}\right)+\frac{\partial}{\partial z}\left(k_{l} \frac{\partial T}{\partial z}\right)+\dot{q}=\rho c_{p} \frac{\partial T}{\partial t}
$$

\subsubsection{Assumptions}

The conduction of heat in the thin film is only considered in the $y$ direction. Note that once the heat is at the surface, the assumption of a uniform vapour field requires that no heat be transferred to the vapour via conduction. As stated for the system at the top level, the conduction is assumed to be steady state. Lastly, the thin film

receives heat from the channel walls and does not generate its own heat. Applying these conditions to Equation (27) to get,

$$
\frac{\partial}{\partial y}\left(k_{l} \frac{\partial T}{\partial y}\right)=0
$$




\subsubsection{Boundary conditions}

The boundary conditions are applied at the liquid-vapour interface and the liquidsolid interface. At the wall, $y=0$,

$$
T=T_{w}
$$

At the liquid-vapour interface, $y=\delta$,

$$
-k_{l} \frac{\partial T}{\partial y}=\dot{m}_{e v a p}^{\prime \prime} h_{f g}
$$

Thus applying boundary conditions (29) and (30) to Equation (28) and integrating we get the following relation for the evaporative mass flux.

$$
\dot{m}_{\text {evap }}^{\prime \prime}=\frac{k_{l}\left(T_{w}-T_{l v}\right)}{\delta h_{f g}}
$$

This evaporative mass flux relation can be related to the mass flow in the thin film through integration. Thus, we can obtain the mass flow rate as:

$$
\dot{m}=-\int_{0}^{x} \frac{k_{l}\left(T_{w}-T_{l v}\right)}{\delta h_{f g}} d x
$$

The change of sign is a result of the mass flow gradient and the coordinate system. 


\subsection{Liquid pressure gradient}

It is now possible to solve for the relation that governs the liquid pressure gradient. This is done by equating the mass flow rates as described by Equations (26) and (32). Solving for the liquid pressure gradient:

$$
\frac{d P_{l}}{d x}=b \frac{C_{5}}{C_{4}} \frac{d T_{l v}}{d x}+\frac{\nu k_{l}}{C_{4} h_{f g}} \int_{0}^{x} \frac{T_{w}-T_{l v}}{\delta} d x
$$

Note this liquid pressure gradient relation is still dependent on the interfacial temperature gradient. To solve for this, another model must be introduced.

\subsection{Kelvin-Clapeyron equation}

To complete the set of equations which describe the evaporation at the liquid-vapour interface, Wayner proposed the use of a Kelvin-Clapeyron model [17].

$$
\dot{m}_{\text {evap }}^{\prime \prime}=\frac{1}{h_{f g}}\left(h_{l v}^{c l}\left(T_{l v}-T_{v}\right)-h_{l v}^{k l}\left(P_{d}+P_{c}\right)\right)
$$

We now have two relations which describe the evaporative mass flux. Thus we can combine Equations (31) and (34). Solving we get a relation which describes the interface temperature as a function of the thin film geometry. More importantly, the interfacial temperature profile is no longer a function of the liquid pressure.

$$
\frac{k_{l}}{\delta \eta} T_{l v}^{5 / 2}-\frac{k_{l}}{\delta \eta} T_{w} T_{l v}^{3 / 2}+\frac{M h_{f g}}{T_{v}} T_{l v}-V_{l} b T_{l v} \kappa=M h_{f g}+V_{l}\left(\frac{A}{\delta^{3}}+a \kappa\right)
$$




\subsection{Interface temperature gradient}

The last part of the model comes from the interface temperature gradient. This is obtained by differentiating Equation (35) with respect to $x$ and collecting terms to give:

$$
\frac{d T_{l v}}{d x}=\left(\frac{2 T_{v}}{\delta^{3}}\right)\left(\frac{\chi+V_{l} \eta \sigma \delta^{4} \kappa^{\prime}}{\omega}\right)
$$

Where,

$$
\begin{gathered}
\chi=\delta^{\prime}\left[k_{l} \delta^{2}\left(T_{l v}^{5 / 2}-T_{w} T_{l v}^{3 / 2}\right)-3 A V_{l} \eta\right] \\
\omega=k_{l} T_{v}\left(5 T_{l v}^{3 / 2}-3 T_{w} T_{l v}^{1 / 2}\right)+2 \delta \eta\left(M h_{f g}-V_{l} T_{v} b \kappa\right)
\end{gathered}
$$

\subsection{Complete Model}

Now it is possible to assemble all of the parts previously derived, to obtain a third order ODE that appropriately describes the profile of the evaporating thin film region. To do this, we must combine Equations (19), (33), (36) and the curvature gradient. Thus we have the final form of the third order ODE, as outlined in Appendix A:

$$
\delta^{\prime \prime \prime}=\frac{3 \delta^{\prime} \delta^{\prime 2}}{\alpha}-\frac{2 T_{v} \psi \chi}{\omega \delta^{3} \lambda}+\frac{\theta}{\lambda}
$$

Where,

$$
\begin{gathered}
\alpha=1+\left(\delta^{\prime}\right)^{2} \\
\theta=\frac{\alpha^{3 / 2}}{\sigma}\left(\frac{3 A}{\delta^{4}} \delta^{\prime}-\frac{\nu k_{l}}{C_{4} h_{f g}} \int_{0}^{x} \frac{T_{w}-T_{l v}}{\delta} d x\right)
\end{gathered}
$$




$$
\begin{gathered}
\psi=\left(\frac{b}{\sigma}\right)\left(\frac{C_{5}}{C_{4}} \alpha^{3 / 2}+\delta^{\prime \prime}\right) \\
\lambda=\left(1+\frac{2 T_{v} \psi V_{l} \eta \sigma \delta}{\omega \alpha^{3 / 2}}\right)
\end{gathered}
$$

\subsection{Initial conditions}

With the complete form of the differential equation, it is possible to solve for the profile of the evaporating thin film. The conditions of the model are that of a set of boundary conditions that must be satisfied. The boundaries are for that of the adsorbed region with a zero curvature, and the bulk region with a constant $1 / r$ curvature. Although a boundary value problem, it is possible to solve the governing differential equation with the application of a set of initial conditions and a shooting technique. As such, the solution to Equation (39) can be obtained with the input of initial values for $\delta, \delta^{\prime}, \delta^{\prime \prime}$ and $T_{l v}$. It is with these initial conditions that all properties of the thin film can be obtained. However for the solution to be valid, the thin film must monotonically increase in thickness with length. As such, the initial derivatives must be greater than or equal to zero. 
The order of magnitude of the initial conditions can be reasoned out by considering the adsorbed thin film region. The adsorbed region is dominated by intermolecular forces, which retain the liquid and prevent evaporation [13]. If the adsorbed region is taken to extend for a distance past the thin film region, it would be of a uniform thickness on the heated channel wall. This would suggest that the curvature in the adsorbed region is zero.

For the case where the thin film coordinate system is placed adjacent to the adsorbed region, all of the film thickness derivatives would be zero. Although mathematically valid, when substituted into Equation (39), results in a trivial solution. To avoid this scenario, the origin is placed near the adsorbed region. This then requires the application of perturbations to the derivatives. Thus we can infer from Equation (18), that the second derivative of film thickness will be near zero.

The thin film region nearest the adsorbed region is expected to be of a comparable thickness to that of the adsorbed region. As such the thickness perturbation is to be small, as too should the first derivative. The size of the perturbations applied must then be based on the scale of the adsorbed film thickness.

\subsection{Adsorbed thickness}

The adsorbed thin film region is assumed to be of a uniform thickness. Of particular importance is the fact that the adsorbed layer does not undergo evaporation due to the attractive intermolecular forces. Thus the thickness of the adsorbed layer can be determined by setting the evaporative mass flux of Equation (34) to zero.

Although this simplifies the Kelvin-Clapeyron model, the temperature jump and capillary pressure are required to solve for the adsorbed layer. From the aforementioned conditions of the adsorbed region, the curvature is assumed to be zero. This then removes the capillary pressure term. Lastly is the interface temperature. As 
the adsorbed layer is very thin, order of nanometers, it may be assumed that the conductive losses are negligible $[5,9]$. Thus the interface temperature can be taken as that of the channel wall. The complete derivation is presented in Appendix B, with the final result of the derivation given as:

$$
\delta_{0}=\left[\frac{A V_{l}}{M h_{f g}}\left(\frac{T_{v}}{T_{w}-T_{v}}\right)\right]^{1 / 3}
$$

\subsection{Initial condition limits}

Despite the logic applied in describing the adsorbed region, it is possible to select values that violate the evaporative mass flux balance. The balance is the same as that of Equation (35). For ease of use, the balance is reformed by equating the evaporative mass flux of Equations (34) and (31).

$$
\frac{k_{l}\left(T_{w}-T_{v}\right)}{\delta h f g}=\frac{\eta}{h_{f g}}\left(\frac{1}{T_{v}}\right)^{3 / 2}[\frac{M h_{f g}}{T_{v}} \underbrace{\left(T_{v}-T_{v}\right)}_{0}-V_{l}\left(P_{d}+P_{c}\right)]
$$

Now it is possible to determine the maximum and minimum values of $\delta^{\prime \prime}$ for a given $\delta$ and $\delta^{\prime}$. A comprehensive form of the derivation is presented in Appendix C.

\subsubsection{Initial condition minimum}

The minimum value of $\delta^{\prime \prime}$ can be determined by applying the minimum interfacial temperature, $T_{v}$. Replacing $T_{l v}$ with $T_{v}$ in Equation (45) and solving for $\delta^{\prime \prime}$.

$$
\delta_{m i n}^{\prime \prime}=-\alpha^{3 / 2}\left[\frac{k_{l}\left(T_{w}-T_{v}\right)}{V_{l} \sigma \delta \eta}\left(T_{v}\right)^{3 / 2}+\frac{P_{d}}{\sigma}\right]
$$


Analyzing each collection of terms, it can be seen that the overall result is a negative minimum limit for $\delta^{\prime \prime}$. Considering the minimum limit and the previously defined requirement for $\delta^{\prime \prime} \geq 0$, the minimum limit for $\delta^{\prime \prime}$ is set to be equal to zero.

\subsubsection{Initial condition maximum}

Conversely the maximum value of $\delta^{\prime \prime}$ can be determined by applying the maximum interfacial temperature, $T_{w}$. Replacing $T_{l v}$ with $T_{w}$ in Equation (45) and solving for $\delta^{\prime \prime}$.

$$
\delta_{\max }^{\prime \prime}=\frac{\alpha^{3 / 2}}{\sigma}\left(\frac{M h_{f g}}{T_{v} V_{l}}\left(T_{w}-T_{v}\right)-P_{d}\right)
$$

Evaluating each set of terms in Equation (47), we observe that each set is greater than zero. This suggests that the overall solution for the maximum value of $\delta^{\prime \prime}$ can be either negative or positive. To ensure that the thin film is of monotonically increasing thickness, the following inequality must be satisfied.

$$
\frac{M h_{f g}}{T_{v} V_{l}}\left(T_{w}-T_{v}\right) \geq \frac{A}{\delta^{3}}
$$




\section{Chapter 4}

\section{Numerical Method of Solution}

The development of the thin film mathematical model resulted in the formulation of a third order differential equation. As this formulation is related to those of previous works $[3,5]$, the nature of this equation was expected to exhibit a highly non-linear behaviour, with sensitivities to the film thickness $(\delta)$ and the first derivative $\left(\delta^{\prime}\right)$. As a result, the method by which the differential equation was solved used a combination of user defined inputs and computational search algorithms.

The differential equation was solved using a constant, user defined, step size. The constant step size provided a less complicated method of solving the differential equation. Although not computationally efficient, it is more stable given the sensitivities of the initial conditions and associated perturbations. Thus the thin film profile is discretely solved over the length, with a resolution defined by the user defined step size. To maintain a high resolution, a step size of one nanometer was used. 


\subsection{The differential equation}

The evaporating thin film portion of a meniscus as described by Equation (39), cannot be simply integrated symbolically or numerically. To circumvent this complication, the third order differential equation was reduced to a set of three first order differential equations. The complete process of reduction is provided in Appendix D, with the final results shown here.

$$
\begin{gathered}
d_{0}^{\prime}=d_{1} \\
d_{1}^{\prime}=d_{2} \\
d_{2}^{\prime}=\frac{3 d_{1} d_{2}^{2}}{\alpha}-\frac{2 T_{v} \psi \chi}{\omega \lambda d_{0}^{3}}+\frac{\theta}{\lambda}
\end{gathered}
$$

With the change of variables and the reduction in order, the set of first order differential equations can be solved using numerical techniques.

\subsection{Algorithm outline}

The formation of a numerical model for the thin film profile, is described by Equations (49), (50) and (51). To achieve a valid solution, an algorithm was developed for which a series of loops and convergence criteria are employed. The general structure of the algorithm is provided in Figure 4, and the inner working of the Runge-Kutta solving section outlined in Figure 5. 


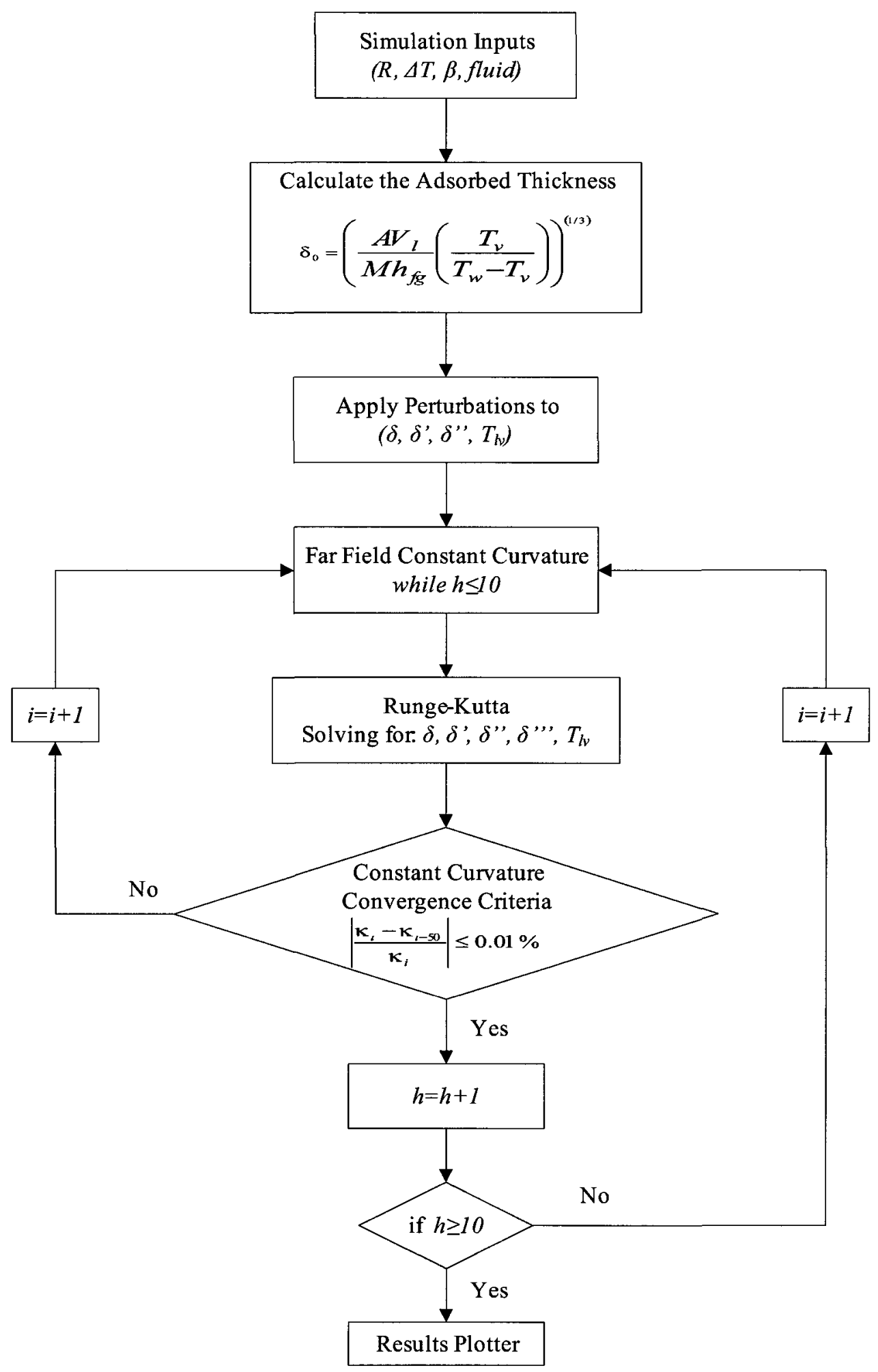

Figure 4: Main algorithm flowchart 


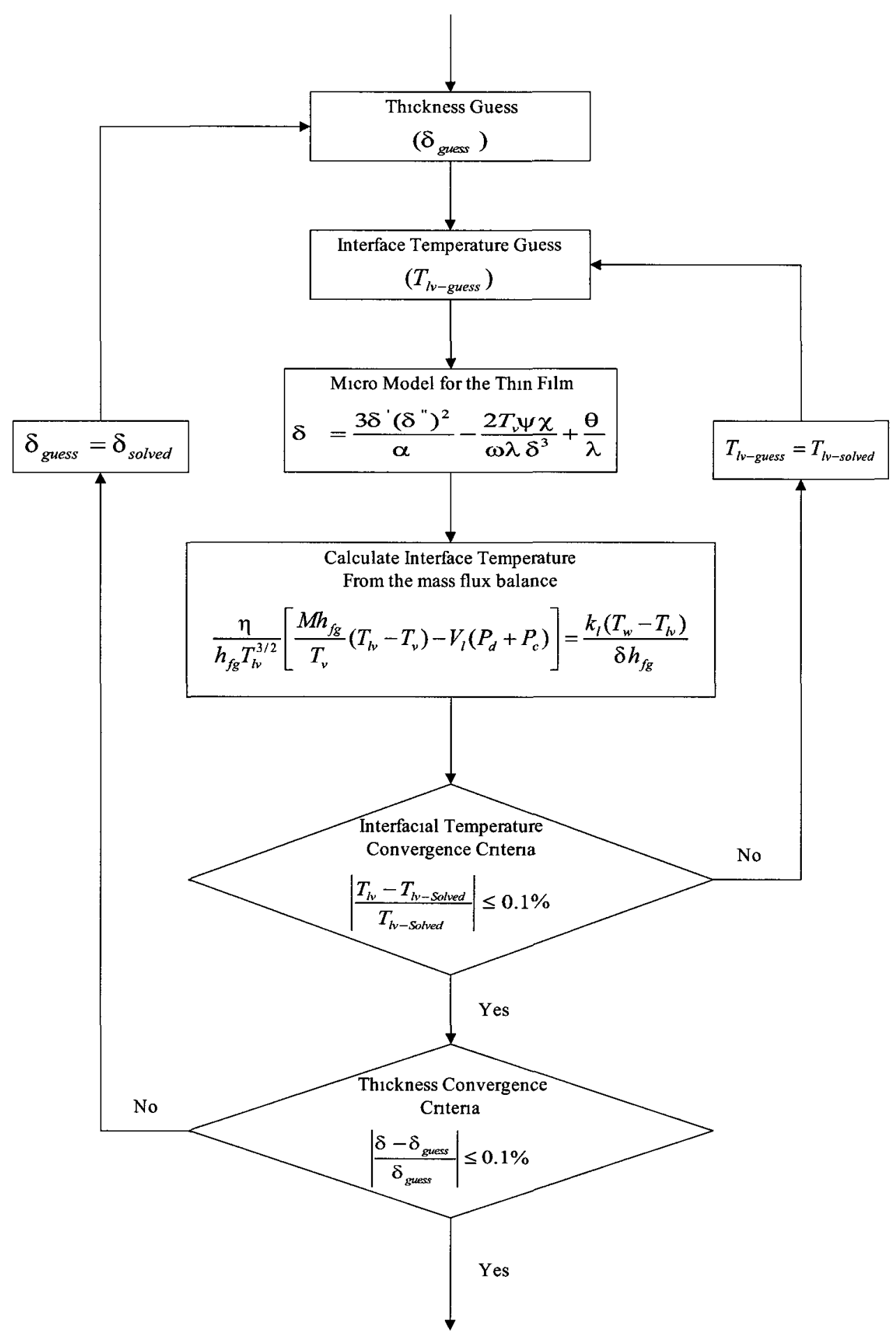

Figure 5: Runge-Kutta algorithm flowchart 


\subsection{Required inputs}

The solution of the differential equations was set up so as to allow for the variation of variables. These variations were selected so as to account for differences in the fluid used, channel geometry, applied superheat, wall slip length and interfacial temperature gradients. With these defined user inputs selected, a set of boundary conditions and initial conditions can be preset for the numerical solution.

\subsection{Initial conditions}

To solve the set of first order differential equations, some particulars are required. As stated previously, the third order differential equation set would ideally use initial conditions such as:

$$
\begin{aligned}
\delta & =\delta_{0} \\
\delta^{\prime} & =0 \\
\delta^{\prime \prime} & =0 \\
T_{l v} & =T_{w}
\end{aligned}
$$

Although the above conditions are mathematically valid, they would result in a trivial solution. This trivial solution would equate to that of a continuous adsorbed layer of infinite length. To avoid the trivial solution, a set of small perturbations can be applied to the film thickness $(\delta)$, first derivative $\left(\delta^{\prime}\right)$ and the second derivative $\left(\delta^{\prime \prime}\right)$. These perturbations are to be small in comparison to the original values, so as to simulate the shift of the coordinate system. This shift is a means of placing the origin within the thin film region and ensuring the formation of a monotonically increasing thin film thickness. 


\subsection{Applied perturbations}

To achieve a solution to the differential equation a set of perturbations are applied to the ideal initial conditions. The magnitude of the perturbations is selected to be greater than zero so as to ensure the growth of the thin film as a function of length. Each of the initial conditions requires a specific perturbation to be defined.

The first initial condition is that of the film thickness $(\delta)$, with the smallest value stated to be equal to that of the adsorbed layer. As discussed by Zhao et al. [3], the film thickness portion of the differential equation is sensitive to perturbations. This sensitivity was not discussed by other researchers, and instead the perturbation was selected to be of an arbitrary length $[5,9]$. To ensure the proximity of the coordinate systems origin to that of the adsorbed region, a small percentage based perturbation was selected. The perturbation was selected to be $0.1 \%$ of the adsorbed thickness.

This method of perturbing the thin film thickness was selected so as to ensure uniformity when changing fluids or applied superheats. The reasoning for this perturbation method becomes apparent with the investigation of the adsorbed layer thickness, as defined previously by Equation (44).

$$
\delta_{0}=\left[\frac{A V_{l}}{M h_{f g}}\left(\frac{T_{v}}{T_{w}-T_{v}}\right)\right]^{1 / 3}
$$

Restating Equation (44) above, it is possible to see that the adsorbed thickness is a function of both the dispersion constant and the applied superheat. Thus when numerically modeled, the perturbation will remain proportionate to the adsorbed thickness. This subsequently removes any inconsistencies in the perturbation thickness as a result in the changing of solid-liquid systems or applied superheats.

The second perturbation to be applied is to that of the first derivative of film thickness $\left(\delta^{\prime}\right)$. With the review of previous works $[3,9]$, the first derivative is sensitive to perturbations, for third order systems. As such it was selected to be the search 
parameter for finding the desired far field curvature. Despite being selected as the search parameter, the expected value is to be positive and small, so as to keep with the goal of proximity to the adsorbed region.

The third perturbation is applied to the second derivative of film thickness $\left(\delta^{\prime \prime}\right)$. This derivate is the dominant term in the curvature definition as previously described by Equation (18). As the origin is to be near the adsorbed region, the second derivative perturbation was selected to be positive and near zero, with a value of $1 \times 10^{-7}$.

The last perturbation is that of the interface temperature $\left(T_{l v}\right)$. As the film thickness near the adsorbed region is close to that of the adsorbed region, it is possible to assume that the temperature difference is extremely small. Thus the interface temperature is expected to be close to that of the wall temperature, as conductive losses are negligible. As such, any perturbations to be applied to the interface temperature will vary between the wall temperature and the vapour temperature.

\subsubsection{Perturbation limits}

In conjunction with the application of perturbations, an underlying limit is present, as described earlier. Provided that the solution is achieved by defining the perturbations applied to $\delta$ and $\delta^{\prime}$, the upper and lower limits for $\delta^{\prime \prime}$ can be determined. As stated in the previous chapter, the lower limit is negative for $\delta^{\prime \prime}$ with the upper limit being greater than zero. Thus, a termination condition was created so as to ensure that $\delta^{\prime \prime}$ did not exceed the maximum value at any point during the numerical modeling. 


\subsection{Parametric study}

Of the few pieces of information found in the literature, the sensitivities of the differential equation $[3,5]$, require some form of verification. Thus, a parametric routine was developed so as to investigate each of the film thickness and associated derivative perturbations. The control aspect of the routine, was the application of a single perturbation variance so as to remove any potential interdependencies. As such a battery of tests could be conducted for a user defined range of perturbations. The results of such tests could then be graphically displayed in a three dimensional plot.

\subsection{The Hamaker constant}

The determination of the Hamaker constant is of great importance, as it allows for the quantification of the disjoining pressure acting within the thin film. Of the previous thin film models covered $[3,5,9,11,12,14-16,19,32]$, none actually determined the value of the Hamaker constant as a function of the liquid-solid system. Instead the value was obtained by simply referencing other works, for which they had referenced a source. To avoid the potential misappropriation of the value, it was decided to make use of the Tabor-Winterton approximation defined previously as Equation (8) [27].

This approximation of the Hamaker constant was selected as it provided a relation between the dielectric constant and index of refraction for each component. Thus it is possible to calculate a value for the Hamaker constant for any given material combination. This method is more consistent in determining the Hamaker constant as opposed to the methods previously used. The last piece of information required to complete the Tabor-Winterton approximation is the determination of the main absorption frequency for the liquid. The main absorption frequency was obtained using absorption data from Zachariasse et al. [27,38,39]. 
Although this method of calculation accounts for more variations in material combinations, it still retains simplifications. These include those of the Tabor-Winterton approximation itself, as well as the assumed single main absorption frequency, the values used for the index of refraction and the dielectric constants. It is important to note that the values used were used over a small range of superheats. This was done so as to mitigate the effects of temperature dependence in the index of refraction or dielectric constant, such as those observed by Catenaccio et al. [40].

\subsection{Adsorbed thickness}

The film thickness in the adsorbed region was assumed to be of a uniform thickness and extended along the channel wall. This assumption is based on the experimental work of Buffone et al. [10,24], and the theoretical works of Wayner Jr. et al. [2,3, 6,10-12]. Thus the adsorbed film thickness is determined using Equation (44). This calculated value, provides a basis for determining the size of the perturbation to be applied.

\subsection{Integral evaluation}

The differential equation which describes the thin film profile is a third order ODE. As discussed earlier, other researchers have used third and fourth order differential equations to describe the profile. It was decided to maintain the governing equation as a third order differential so as to avoid any information loss. 
The governing equations as derived earlier, resulted in a third order differential equation with an integration term contained within the liquid pressure gradient, Equation (33). Isolating the integration term in Equation (33),

$$
\int_{0}^{x} \frac{T_{w}-T_{l v}}{\delta} d x
$$

The integral is evaluated at each point $x$, with a cumulative total running from the adsorbed region $(x=0)$ to the current point $(x=x)$. Thus the solution of the thin film profile requires knowledge of the film thickness and interface temperature from the adsorbed region through to the current point of interest.

\subsubsection{Thin film thickness}

The thin film thickness value to be used in the integral is an unknown and is to be solved for. Thus an iterative technique can be employed where the value of the film thickness is given a placeholder value, near to the expected value. To estimate the film thickness value, the value from the adjacent point is used as a first approximation to the current value.

$$
\delta_{i}=\delta_{i-1}
$$

\subsubsection{Interfacial temperature}

For the interface temperature, the same method as that of the film thickness was used. Thus the interface temperature value is set to that of the value adjacent, as a temporary place holder.

$$
T_{l v_{i}}=T_{l v_{i-1}}
$$




\subsubsection{Integral total}

The values chose for $\delta$ and $T_{l v}$, will allow for the solving of the governing differential Equation (39), as it will provide a numerical value to replace the integral described by Equation (56). Upon solving of the current step, the values calculated for $\delta$ and $T_{l v}$ will be compared with the guessed values used as place holders. Any differences within a specified tolerance result in the re-evaluation of the current step until the values are below the prescribed tolerance. It is for this reason that the values are selected initially to be that of the adjacent point so as to minimize the number of iterations.

The starting condition of the integral value can be determined using the assumptions used in the derivation of the governing Equation (39). Thus we get the initial integral value nearest the adsorbed region.

$$
\int_{0}^{x} \frac{T_{w}-T_{w}}{\delta_{0}} d x=0
$$

The value of the integral in the adsorbed region is assumed to be zero as a consequence of the interfacial temperature assumption. This assumption states that the interface temperature is to be equal to the wall temperature due to the negligible conductive loss across the adsorbed film thickness.

Lastly is the cumulative total of the integration. This is achieved through the following integral equivalence,

$$
\int_{0}^{x} \frac{T_{w}-T_{l v}}{\delta} d x=\underbrace{\int_{0}^{x_{\imath-1}} \frac{T_{w}-T_{l v}}{\delta} d x}_{\text {Previous integral total }}+\underbrace{\int_{x_{\imath-1}}^{x_{\imath}} \frac{T_{w}-T_{l v}}{\delta} d x}_{\text {Current integral }}
$$


The previous integral total portion of Equation (60) represents the cumulative total of all points previous to the current, and is recorded as a value for each progressive step. However for the current integral term part of Equation (60), this value is calculated for each successive step and summed with the previous integral total.

The current integral portion of Equation (60), is evaluated using the trapezoidal rule as given below by Equation (61) [41].

$$
\int_{x_{\imath-1}}^{x_{\imath}} \frac{T_{w}-T_{l v}}{\delta} d x \approx\left(x_{\imath}-x_{\imath-1}\right)\left(\frac{\left(\frac{T_{w}-T_{l v}}{\delta}\right)_{\imath}+\left(\frac{T_{w}-T_{l v}}{\delta}\right)_{\imath-1}}{2}\right)
$$

\subsection{Runge-Kutta Solution}

The collection of information and values from all previous steps, allow for the solution of the governing differential, Equation (39). As presented earlier, several methods exist by which to solve a non-linear differential equation. The dominant method used is that of a Runge-Kutta method [3,5,9,14,15,32]. This method was chose as a means of solving the differential equation, as the governing equation is now in the form of an initial value problem.

In keeping with the previous works of Wang et al. [5], the method proposed by Dormand and Prince [42] was used. The difference in the order of the differential equation which governs the thin film, requires the application of a different set of Runge-Kutta coefficients. As the governing equation is third order, it is possible to use two different sets of coefficients, as both will solve the differential equation. The two matrix coefficients are give in Table 1 and 2, as given by Dormand and Prince [42]. 
Table 1: Coefficients for RK5(4)7M

\begin{tabular}{|c|c|c|c|c|c|c|c|c|}
\hline$c_{l}$ & \multicolumn{6}{|c|}{$a_{\imath \jmath}$} & $\hat{b}_{\imath}$ & $b_{2}$ \\
\hline 0 & 0 & 0 & 0 & 0 & 0 & 0 & $\frac{35}{384}$ & $\frac{5179}{57600}$ \\
\hline$\frac{1}{5}$ & $\frac{1}{5}$ & 0 & 0 & 0 & 0 & 0 & 0 & 0 \\
\hline$\frac{3}{10}$ & $\frac{3}{40}$ & $\frac{9}{40}$ & 0 & 0 & 0 & 0 & $\frac{500}{1113}$ & $\frac{7571}{16695}$ \\
\hline$\frac{4}{5}$ & $\frac{44}{45}$ & $-\frac{56}{15}$ & $\frac{32}{9}$ & 0 & 0 & 0 & $\frac{125}{192}$ & $\frac{393}{640}$ \\
\hline$\frac{\delta}{a}$ & $\frac{19372}{6561}$ & $-\frac{25360}{2187}$ & $\frac{64448}{6561}$ & $-\frac{212}{729}$ & 0 & 0 & $-\frac{2187}{6784}$ & $-\frac{92097}{339200}$ \\
\hline 1 & $\frac{9017}{3168}$ & $-\frac{355}{33}$ & $\frac{46732}{5247}$ & $\frac{49}{176}$ & $-\frac{5103}{18656}$ & 0 & $\frac{11}{84}$ & $\frac{187}{2100}$ \\
\hline 1 & $\frac{35}{384}$ & 0 & $\frac{500}{1113}$ & $\frac{125}{192}$ & $-\frac{2187}{6784}$ & $\frac{11}{84}$ & 0 & $\frac{1}{40}$ \\
\hline
\end{tabular}

Table 2: Coefficients for RK5(4)6M

\begin{tabular}{|c|c|c|c|c|c|c|c|}
\hline$c_{\imath}$ & \multicolumn{5}{|c|}{$a_{\imath \jmath}$} & $\hat{b}_{z}$ & $b_{\imath}$ \\
\hline 0 & 0 & 0 & 0 & 0 & 0 & $\frac{19}{216}$ & $\frac{31}{540}$ \\
\hline$\frac{1}{5}$ & $\frac{1}{5}$ & 0 & 0 & 0 & 0 & 0 & 0 \\
\hline$\frac{3}{10}$ & $\frac{3}{40}$ & $\frac{9}{40}$ & 0 & 0 & 0 & $\frac{1000}{2079}$ & $\frac{190}{297}$ \\
\hline$\frac{3}{5}$ & $\frac{3}{10}$ & $-\frac{9}{10}$ & $\frac{6}{5}$ & 0 & 0 & $-\frac{125}{216}$ & $-\frac{145}{108}$ \\
\hline$\frac{2}{3}$ & $\frac{226}{729}$ & $-\frac{25}{27}$ & $\frac{880}{729}$ & $\frac{55}{729}$ & 0 & $\frac{81}{88}$ & $\frac{351}{220}$ \\
\hline 1 & $-\frac{181}{270}$ & $\frac{5}{2}$ & $-\frac{266}{297}$ & $-\frac{91}{27}$ & $\frac{189}{55}$ & $\frac{5}{56}$ & $\frac{1}{20}$ \\
\hline
\end{tabular}


The Runge-Kutta matrix coefficients for both Table 1 and 2 were coded into a MATLAB subroutine. A simple test case of a mass spring system was simulated with the Runge-Kutta solver and MATLAB's ode45. The results were compared with no difference that could be directly observed in the plots. Given the stability of the different Runge-Kutta formulations and plots given by Dormand and Prince [42], the coefficients of Table 1 were used in the Runge-Kutta solver. This subroutine was set up such that the coefficient matrix was pre-defined, along with the restriction of a constant step size to be specified by the user. Finally the Runge-Kutta solver would run such that, it would call each of the first order differential Equations (49), (50) and (51), then solve for the values $\delta_{\imath}, \delta_{\imath}^{\prime}, \delta_{\imath}^{\prime \prime}$ and $\delta_{\imath}^{\prime \prime \prime}$.

\subsubsection{Interface temperature}

The solution of Equation (39) now provides a set of values by which we can solve for the interface temperature. Using evaporative mass flux balance given by Equation (35), the interface temperature can be obtained by using a root finding method. The limits of the interface temperature will be between that of the bulk vapour temperature and the wall temperature. Provided the applied superheat is low, the brackets for a bisection search can be set.

The interface temperature is calculated using the intermediate values from the Runge-Kutta solver. This portion makes up the inner most loop for which the interface temperature value used in the integration term is solved, for Equation (56). For the first pass through the Runge-Kutta solver, the information returned is based on the estimated values of $\delta$ and $T_{l v}$. The interface temperature calculated from the first pass through the Runge-Kutta solver is compared with the guess value of $T_{l v_{\imath-1}}$. If the difference between the calculated and guessed values is too large, the calculated temperature is supplied to the integral term as the new guess. This process continues until the convergence criteria is met. 


\subsubsection{Film thickness}

The guess value used in the integral term, Equation (56), also requires the input of the film thickness at the current point in the solution. Provided the interface temperature has been solved at the current point, from the inner most loop, the film thickness can be evaluated. This is done by a direct comparison of the guess value of $\delta_{\imath-1}$ and the value of $\delta$ solved by the Runge-Kutta solver. If the difference in film thickness values is too large, the film thickness guess is updated using the value of $\delta$ calculated by the Runge-Kutta solver. This process continues until the difference between the guessed and solved values is sufficiently below the convergence criteria.

\subsection{Convergence criteria}

As the program employs a series of loops, some form of convergence criteria are needed. The convergence criteria for each loop allow for the solving of the values of $\delta$ and $T_{l v}$ within the Runge-Kutta solver. So as to avoid the divergence of the solution, the convergence criteria are small with respect to each of the measures.

\subsubsection{Interface temperature}

The interface temperature is calculated in the inner most loop of the Runge-Kutta solver. As the applied superheats are small and the resolution is that of a nanometer scale, the convergence criteria was set to be $0.1 \%$. Thus the error as compared to the convergence criteria is calculated using Equation (62).

$$
\left|\frac{T_{l v_{\text {guess }}}-T_{l v_{\text {solved }}}}{T_{l v_{\text {solved }}}}\right| \times 100 \leq 0.1 \%
$$




\subsubsection{Film thickness}

The second loop used in the Runge-Kutta solver is outside that of the interface temperature loop. The convergence criterion was set to $0.1 \%$, so as to minimize the accumulation of errors. Thus the film thickness error is compared to the convergence criteria using Equation (63).

$$
\left|\frac{\delta_{\text {solved }}-\delta_{\text {guess }}}{\delta_{\text {guess }}}\right| \times 100 \leq 0.1 \%
$$

\subsection{Constant curvature}

With the completion of each step forward, the data collected regarding the film thickness and derivatives was used to calculate the curvature at the current point in the solution. The curvature is calculated throughout the process, so as to determine the point by which the curvature reaches a near constant value. The constant curvature is meant to represent the transition from the thin film region to that of the bulk meniscus region. Thus it is important for the model to determine the point where the transition occurs.

The constant curvature condition is started 75 steps beyond the starting region of the thin film. This was done so as to avoid a premature convergence as the thin film curvature in the region nearest the adsorbed region, has a near constant curvature. Once the scheme has exceeded the 75 steps at the start, a check is performed to determine the curvature and compare it to that of a curvature calculated previous. The curvature comparison uses the currently calculated curvature value and compares it with that of a curvature calculated ten points previous. This comparison is completed using Equation (64). 


$$
\left|\frac{\kappa_{\imath}-\kappa_{\imath-10}}{\kappa_{\imath}}\right| \times 100 \leq 0.01 \%
$$

The curvature of the thin film was to considered constant if the condition described by Equation (64) was satisfied at least ten steps. This is done so as to ensure the curvature is constant for twenty points. The value of twenty comes from the ten point spread used in the constant curvature calculation, and ten points for the constant curvature counter.

In addition to the constant curvature condition, a second condition was imposed. This condition is meant to ensure that the constant curvature counter is counting consecutive points. That is to say that if a constant curvature is achieved for five points and no more, the counter will reset to zero. This ensures the curvature achieved is both a constant value for the twenty point spread and all points are consecutive.

The last curvature convergence condition imposed, ensures the curvature does not become less than zero. A less than zero curvature is associated with that of a decreasing film thickness with length. This condition is not observed in steady state menisci, and therefore a termination command is used. In the event that the curvature of the thin film becomes negative before reaching a constant value, the solver stops, and displays the information to the user regarding the nature of the premature code termination. 


\subsection{Far field curvature}

With the completion of the numerical scheme, a full solution with a constant curvature condition is now available. The curvature condition is the final part which must be sorted out. The far field curvature condition is desired to approach that of the YoungLaplace solution of $1 / r$ or the equivalent $2 / H$. This constant curvature condition is meant to represent that of an ideal solution as described by the Young-Laplace Equation (1).

Provided the numerical scheme has achieved a solution of constant curvature for the far field of the thin film, it can now be compared with that of the desired value of $1 / r$. If the value is greater or less than the desired curvature value, a hand search is conducted to find the opposite. This is done so as to create a bracket set, by which a bisection search can be performed. The bisection search used, determines the appropriate value of $\delta^{\prime}$ needed to get a $1 / r$ far field constant curvature.

Although other more efficient methods exist for the search algorithm, such as a secant search algorithm, the sensitivity of the differential equation proved too great for the algorithm to operate effectively. This is compounded by the requirement for all film thickness values and associated derivatives to be greater than zero. This limits the secant search algorithm to values greater than zero.

\subsection{Complete model}

With the completion of the numerical scheme, a set of results are obtained. The results of the simulation are then plotted so as to illustrate the locations and magnitudes of user selected characteristics. Of these characteristics, the user can select any desired result from those listed in Table 3. 
Table 3: Available results for graphical plotting

\begin{tabular}{|c|c|}
\hline Symbol & Description \\
\hline$\delta$ & Thin film thickness \\
\hline$\delta^{\prime}$ & Thin film slope \\
\hline$\delta^{\prime \prime}$ & Thin film second derivative \\
\hline$\delta^{\prime \prime \prime}$ & Thin film third derivative \\
\hline$\kappa$ & Thin film curvature \\
\hline$\kappa^{\prime}$ & Thin film curvature gradient \\
\hline $\int_{x_{\imath-1}}^{x_{\imath}}\left(\frac{T_{w}-T_{l v}}{\delta}\right) d x$ & Integral term \\
\hline $\int_{x_{0}}^{x_{\imath}}\left(\frac{T_{w}-T_{i v}}{\delta}\right) d x$ & Integral term total \\
\hline$\dot{m}_{\text {evap }}^{\prime \prime}$ & Evaporative mass flux \\
\hline$P_{c}$ & Capillary pressure \\
\hline$P_{d}$ & Disjoining pressure \\
\hline$P_{l}$ & Liquid pressure \\
\hline$P_{\text {comp }}$ & Composite pressure profiles \\
\hline$\frac{d P_{l}}{d x}$ & Liquid pressure gradient \\
\hline$\sigma$ & Surface tension \\
\hline$T_{l v}$ & Interface temperature \\
\hline$\frac{d T_{l v}}{d x}$ & Interfacial temperature gradient \\
\hline
\end{tabular}




\subsection{Sources of error}

The development of the numerical model was carefully done so as to avoid any significant forms of errors. Some of the models developed previously made use of discretized forms of differentials for terms like the interface temperature gradient or pressure gradients $[11,15,16]$. As a means of minimizing these types of discretized errors, the differentials, where possible, were evaluated algebraically.

The numerical model has some forms of error that must be acknowledged. The first and possibly largest error source is the application of perturbations to the initial conditions. To minimize this error, the assumptions reasoned out at the start of the derivation will help to minimize the effects on the final results.

Another form of error observed in the model is the potential for rounding errors in the calculations. This rounding error was addressed by the repeated overworking of the program code so as to formulate a calculation scheme that minimized the number of compounding calculations. It is this reason that lead to the concise form of the governing differential equation. This form as compared with those developed in other works, has removed discretization assumptions and procedural complications.

With regards to the iterative format by which the thin film thickness and interface temperature are obtained is of interest. Although this technique reduces the potential in the loss of information, it introduced some form of error in calculating the film thickness and interface temperature. The convergence criteria set ensures that the errors are small, but however as the Runge-Kutta solver uses data from the previous point to determine the next value. This results in compounding errors in the final results. 


\section{Chapter 5}

\section{Numerical Results}

The mathematical model representing the thin film region of the evaporating meniscus was solved using MATLAB. The results provided here will graphically represent the observed trends in the model and results. The trends and results will then be compared with that of previous works.

The first objective is to confirm that the numerical model matches up with the basic assumptions made in the derivation. Thus a basic simulation for pentane will provide information as to the magnitude and position of the thin film and evaporation region. This is followed by the investigation of uniqueness and parametric studies. These studies will illustrate the highly non-linear behaviour of the third order dif-

ferential equation. As well as provide information regarding the sensitivities of the applied perturbations. 
The second part of the model investigation deals with the effects of wall slip and Marangoni flow. These two aspects were investigated so as to determine how they affect the shape and position of the thin film profile. The remaining results presented are those of geometry, fluids, and operating conditions. The principal results sought for each case include:

- film thickness $(\delta)$

- curvature $(\kappa)$

- evaporative mass flux $\left(\dot{m}_{\text {evap }}^{\prime \prime}\right)$

- interface temperature $\left(T_{l v}\right)$

- liquid pressure $\left(P_{l}\right)$

- capillary pressure $\left(P_{c}\right)$

- disjoining pressure $\left(P_{d}\right)$

- liquid pressure gradient $\left(\frac{d P_{l}}{d x}\right)$ 


\subsection{Basic results}

The numerical model was first run as a means of confirming the results with that of expected trends. The specifics used in the simulation are given in Table 4. The value for the first derivative perturbation is listed as the value solved by the bisection search algorithm. The numerical results are given graphically in Figures 6 through 14. The inputs used allow for some comparisons to the numerical results of Wee et al. [9].

Table 4: Numerical model input values

\begin{tabular}{|l|c|}
\hline Input & Value \\
\hline Superheat & $0.01 \mathrm{~K}$ \\
Vapour temperature & $300 \mathrm{~K}$ \\
Channel radius & $10 \mu \mathrm{m}$ \\
$\epsilon_{1}$ & $4.2 \times 10^{-10}$ \\
$\epsilon_{2}$ & $9.9944 \times 10^{-4}$ \\
$\epsilon_{3}$ & $1 \times 10^{-3}$ \\
$\epsilon_{4}$ & 0 \\
\hline
\end{tabular}




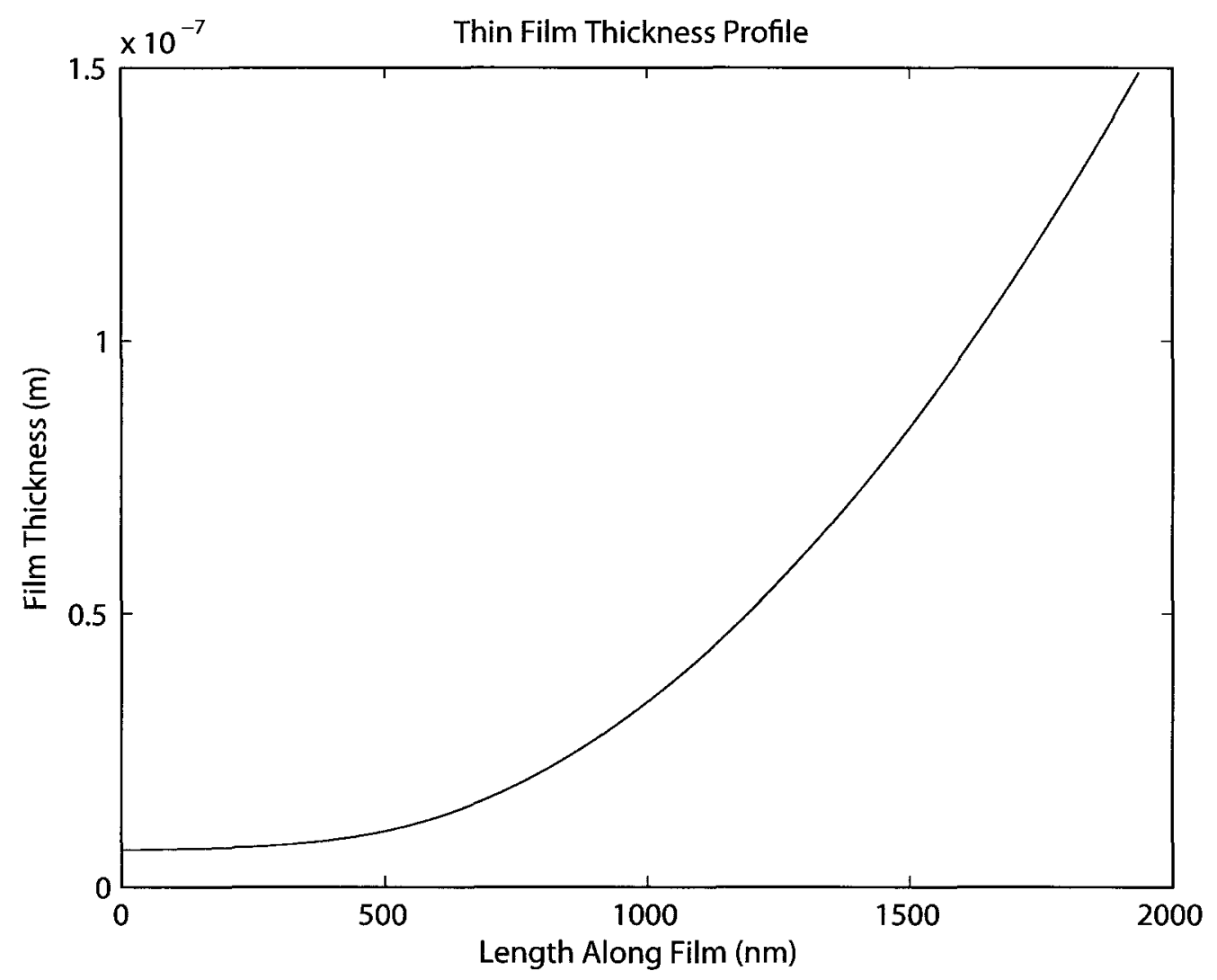

Figure 6: Thin film thickness

The physical thickness of the thin film, Figure 6, progresses monotonically from the adsorbed region to that of the bulk meniscus region. The point of termination as described earlier, is defined as the point by which the curvature is constant for twenty consecutive points within a specified tolerance. Thus from the end of the thin film thickness, the thickness is described by the Young-Laplace Equation (1).

In comparing the values given by the numerical model to those as stated by Wee et al. [9], a difference in overall thin film length is observed. Although different in overall length the values remain within the order of magnitude of those published by Wee et al. [9]. 


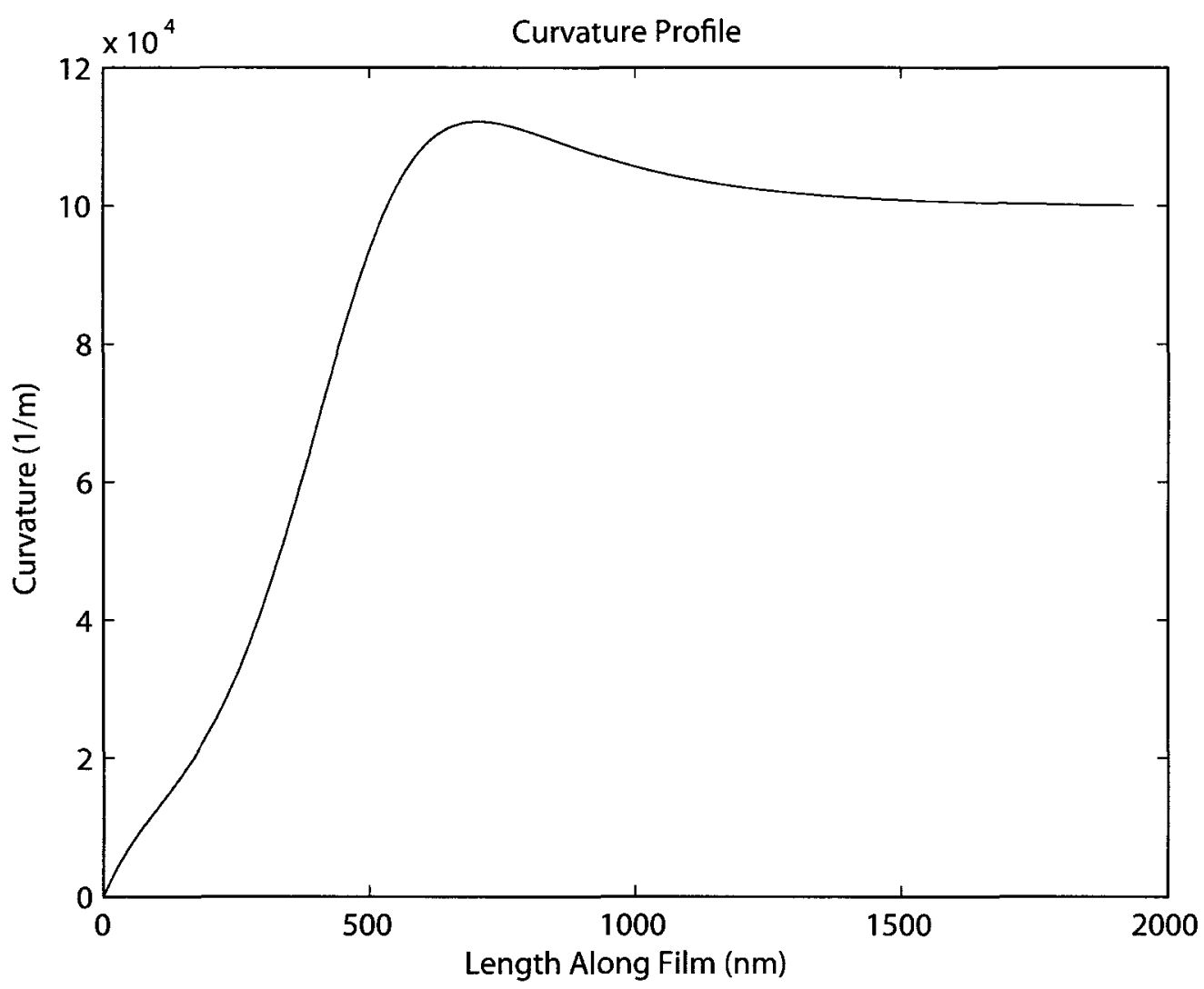

Figure 7: Local curvature

The curvature along the length of the thin film, as shown in Figure 7, is continuous and plateaus in the far end. This curvature profile starts near zero as expected, and climbs rapidly, resulting in some overshoot. Then the curvature recovers and settles to near constant in the far field. The value of the curvature is equal to $1 / r$ with a tolerance of $0.01 \%$. The overshoot characteristic was confirmed by the experimental works of Panchamgam et al. [16]. 


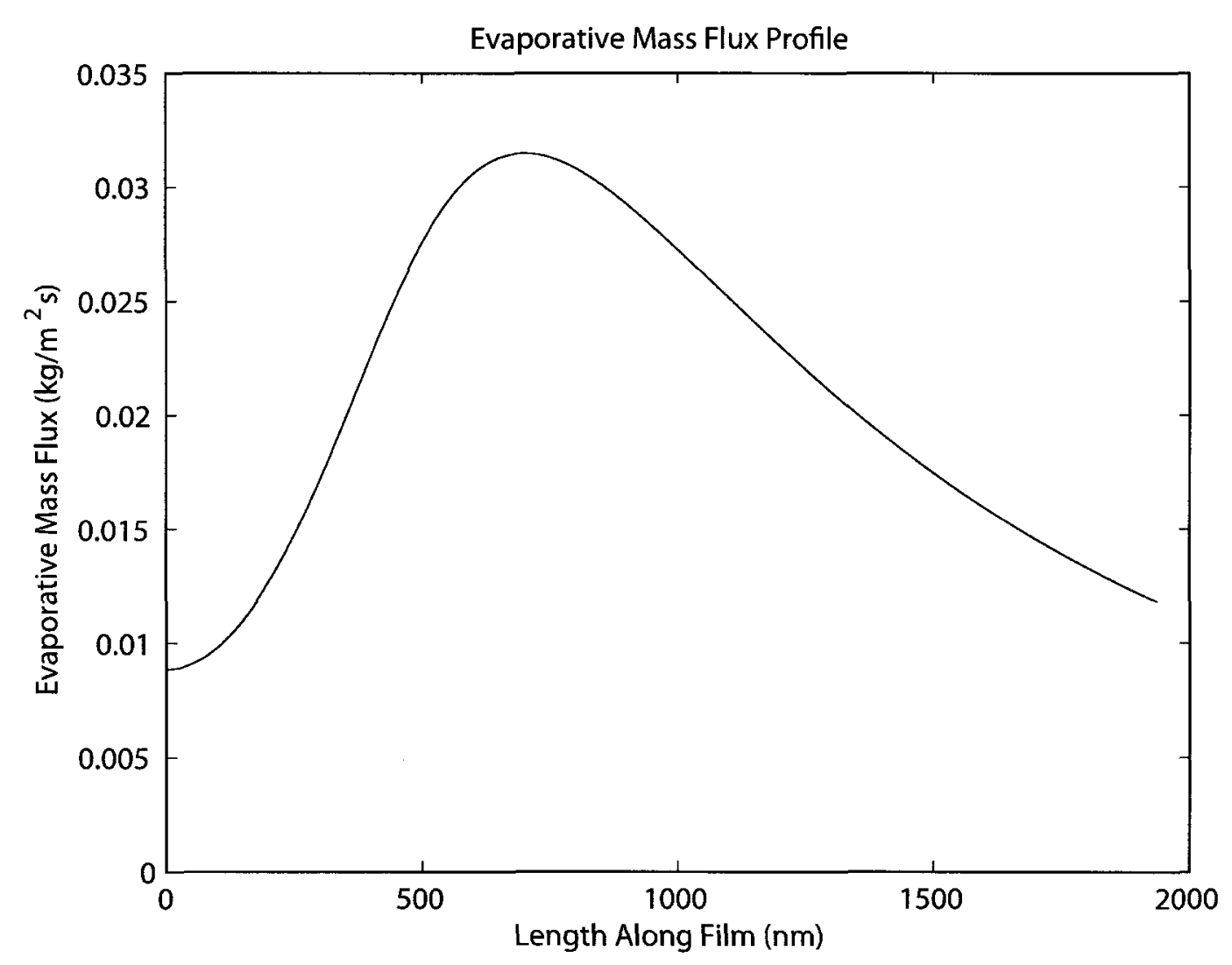

Figure 8: Evaporative mass flux

Of particular importance, is the evaporative mass flux in the thin film region. With the given applications to heat transfer, it is important to determine if the evaporative mass flux varies in this region. As shown in Figure 8, the thin film has some evaporation occurring nearest to the adsorbed region. The non-zero value is a result of the perturbations, as the coordinate system is slightly shifted from the transition point. As the thin film grows in thickness, it undergoes a significant change in evaporative mass flux in the early half of the thin film length. This is followed by the gradual decline in evaporative flux as it transitions into the bulk meniscus.

This decrease in the evaporative mass flux nearest the bulk region, relates to the assumption of the Young-Laplace far field solution. The Young-Laplace solution is for that of a steady state meniscus, where no evaporation is to occur. Given the 
low evaporation $\left(\dot{m}_{\text {evap }}^{\prime \prime} \leq 0.01 \mathrm{~kg} / \mathrm{m}^{2} \mathrm{~s}\right)$, and continued decline, this assumption of a Young-Laplace solution is reasonable. The peak magnitude of the evaporation as compared with Wee et al. [9] is approximately within $85 \%$ of that observed in Figure 8.

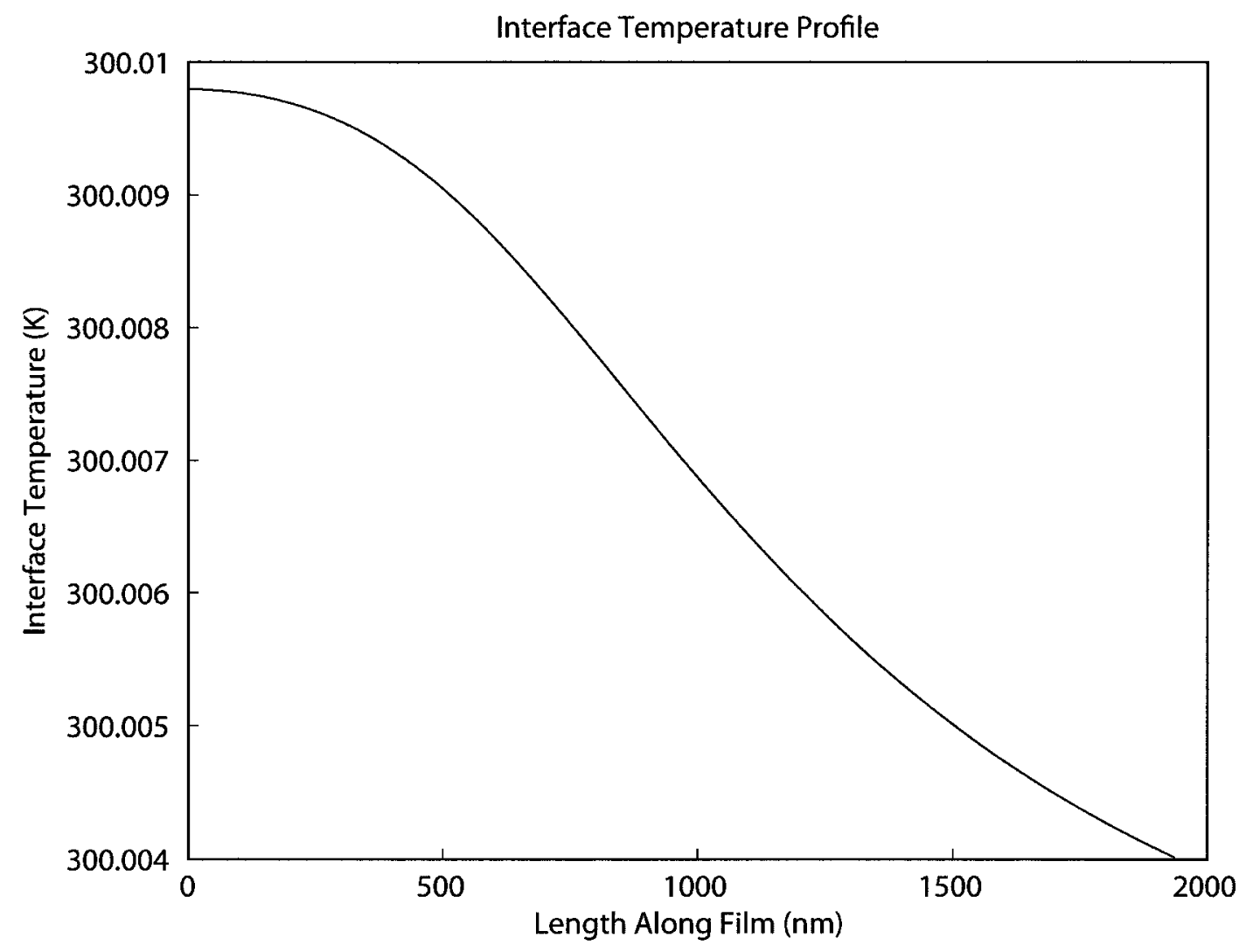

Figure 9: Local interface temperature

The interface temperature along the thin film region, as given in Figure 9, decreases with increasing film thickness. This trend is expected, given the conductive losses and the cooling effect from the evaporation. The monotonic decrease in temperature is typical of numerical models [5,32]. The experimental works of Panchamgam et al. [16,35] and Sodtke et al. [1], have shown a significant temperature dip in the region where evaporation is expected to be highest. 


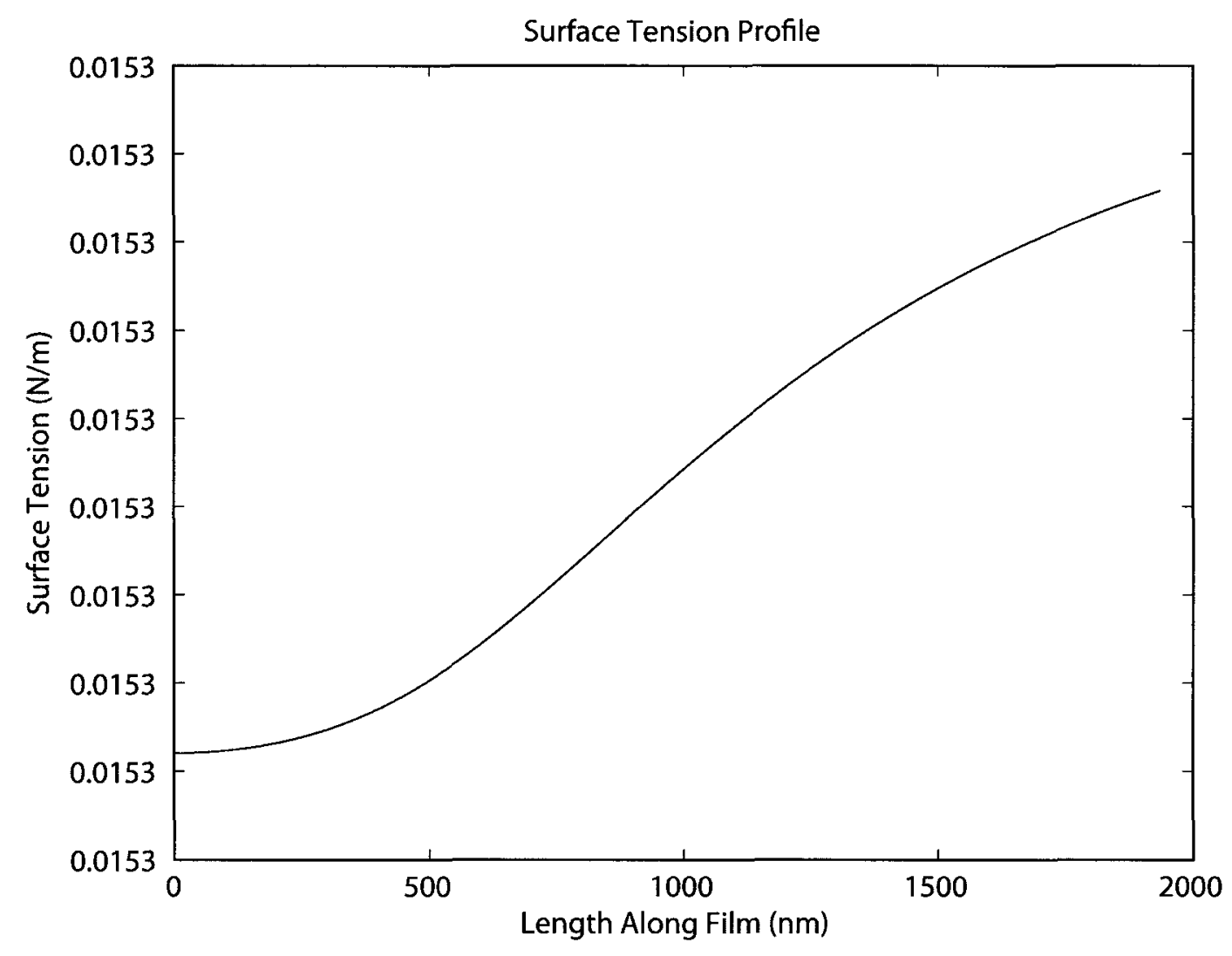

Figure 10: Local surface tension

As a means of confirming the model is working correctly, Figure 10 shows the trend in the surface tension. The trend keeps with the temperature profile as expected, and given the low temperature drop along the length of the thin film, the surface tension gradient is too small to induce any significant Marangoni effects. 


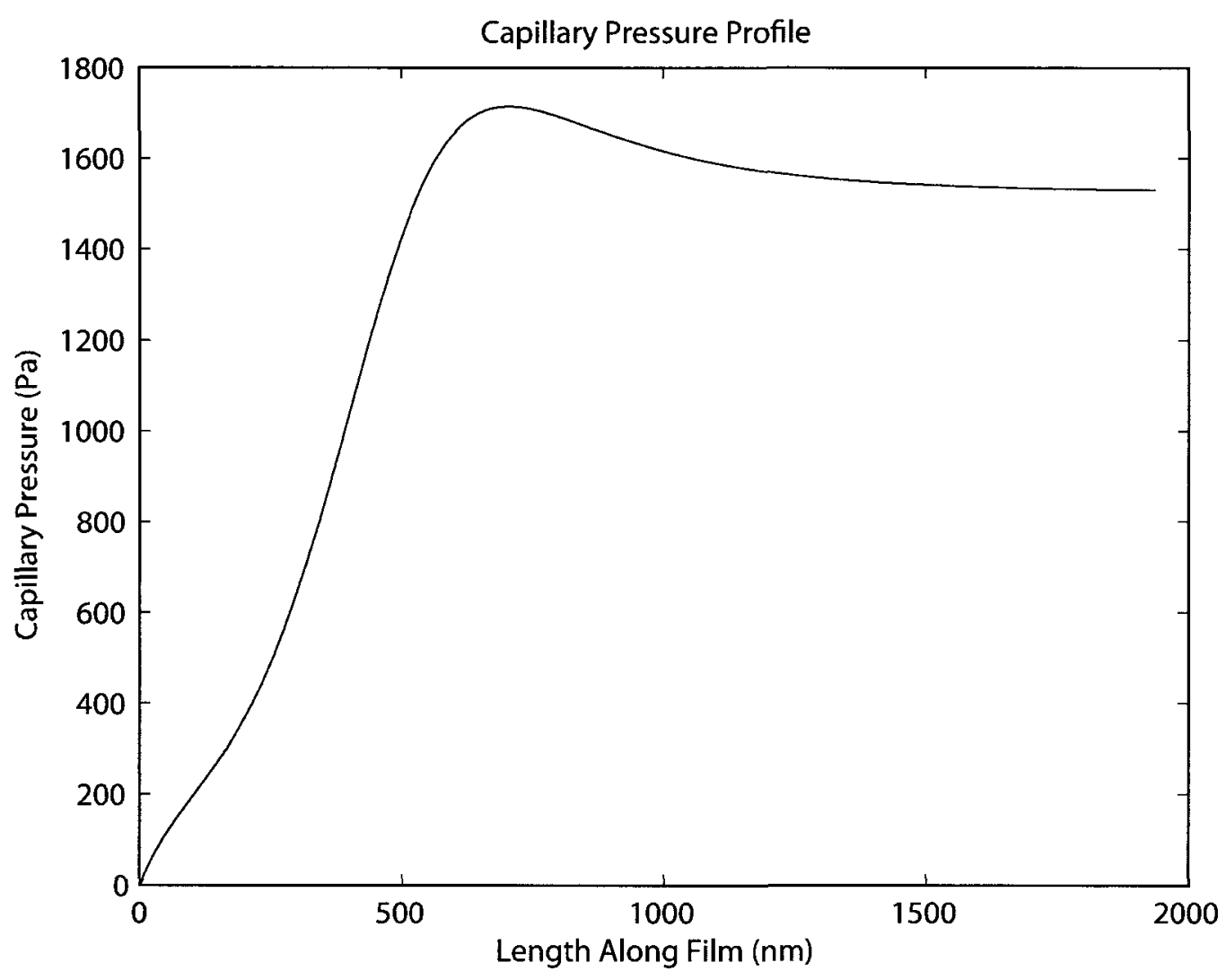

Figure 11: Capillary pressure profile

The capillary pressure acting within the thin film region, shown in Figure 11, provides insight into the transition of pressure dominance. At the start of the thin film region the curvature is near zero and thus so too is the capillary pressure, giving way to the dominance of disjoining pressure. As the thin film grows, the subsequent change in curvature gives way to the rise in capillary pressure. 


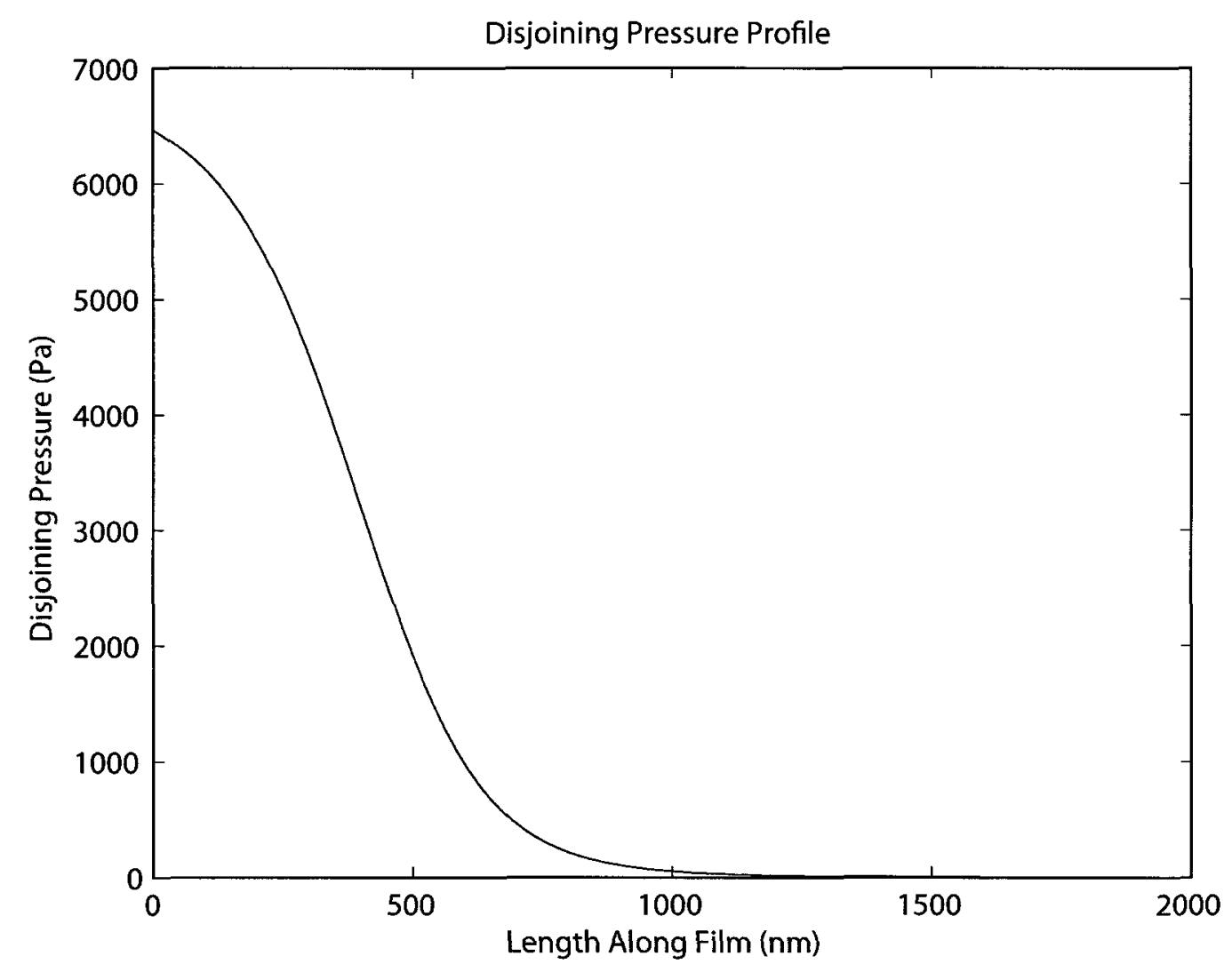

Figure 12: Disjoining pressure profile

The effects of disjoining pressure in the thin film region are of the same importance as those of capillary pressure. The results given in Figure 12, show the expected decay of the disjoining pressure with increase in film thickness. The rapid decline in pressure is observed to occur in the region where the evaporative mass flux is highest. This trend appears accurate as compared with other numerical results $[5,12,15]$. 


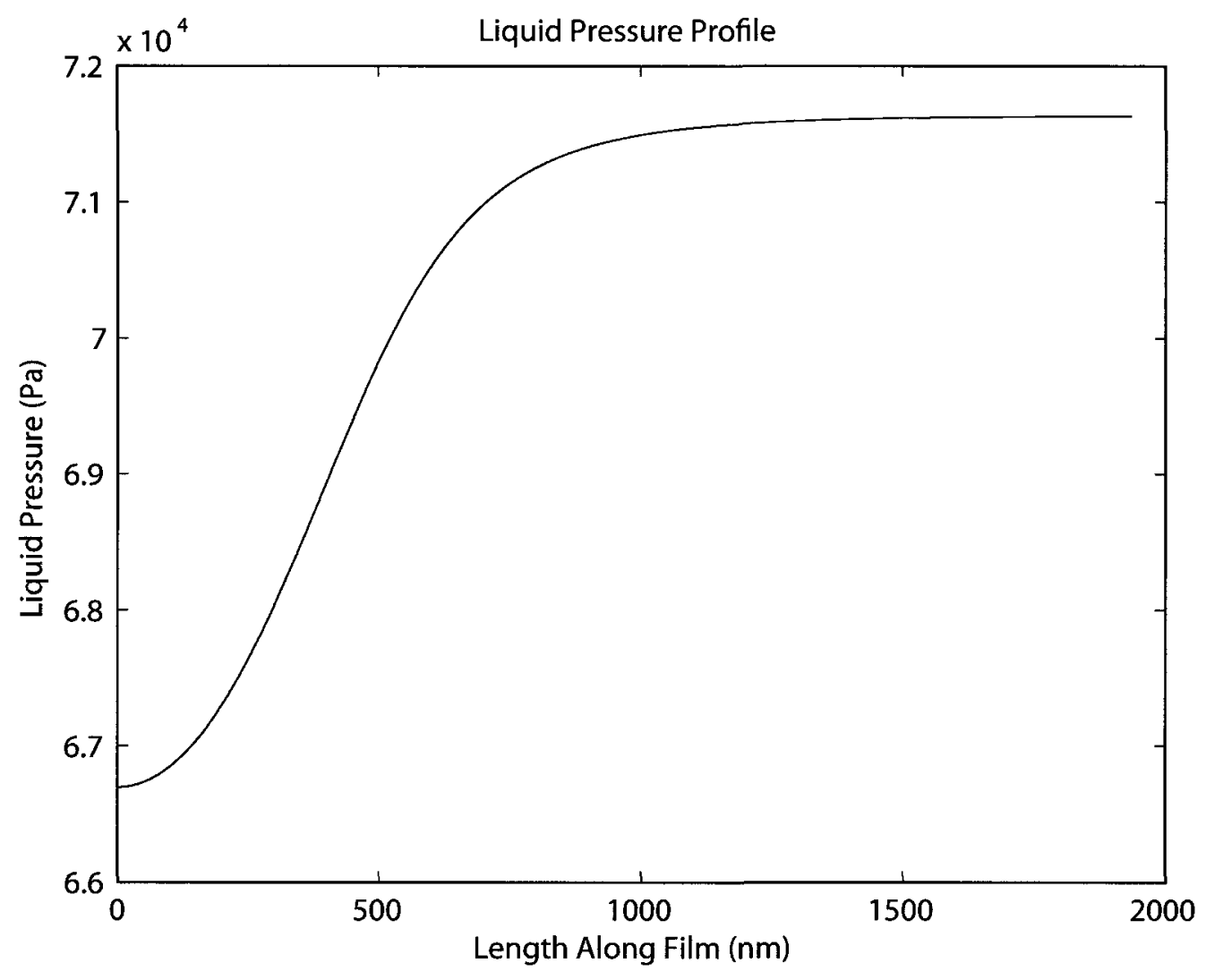

Figure 13: Liquid pressure profile

The liquid pressure profile as given in Figure 13, completes the last of the variances in the pressures acting on the thin film. This pressure comes from the mass flow rate and associated velocities within the liquid thin film. The general shape and trend is the same as that published by Wee et al. [9], with the start and end values being nearly identical. 


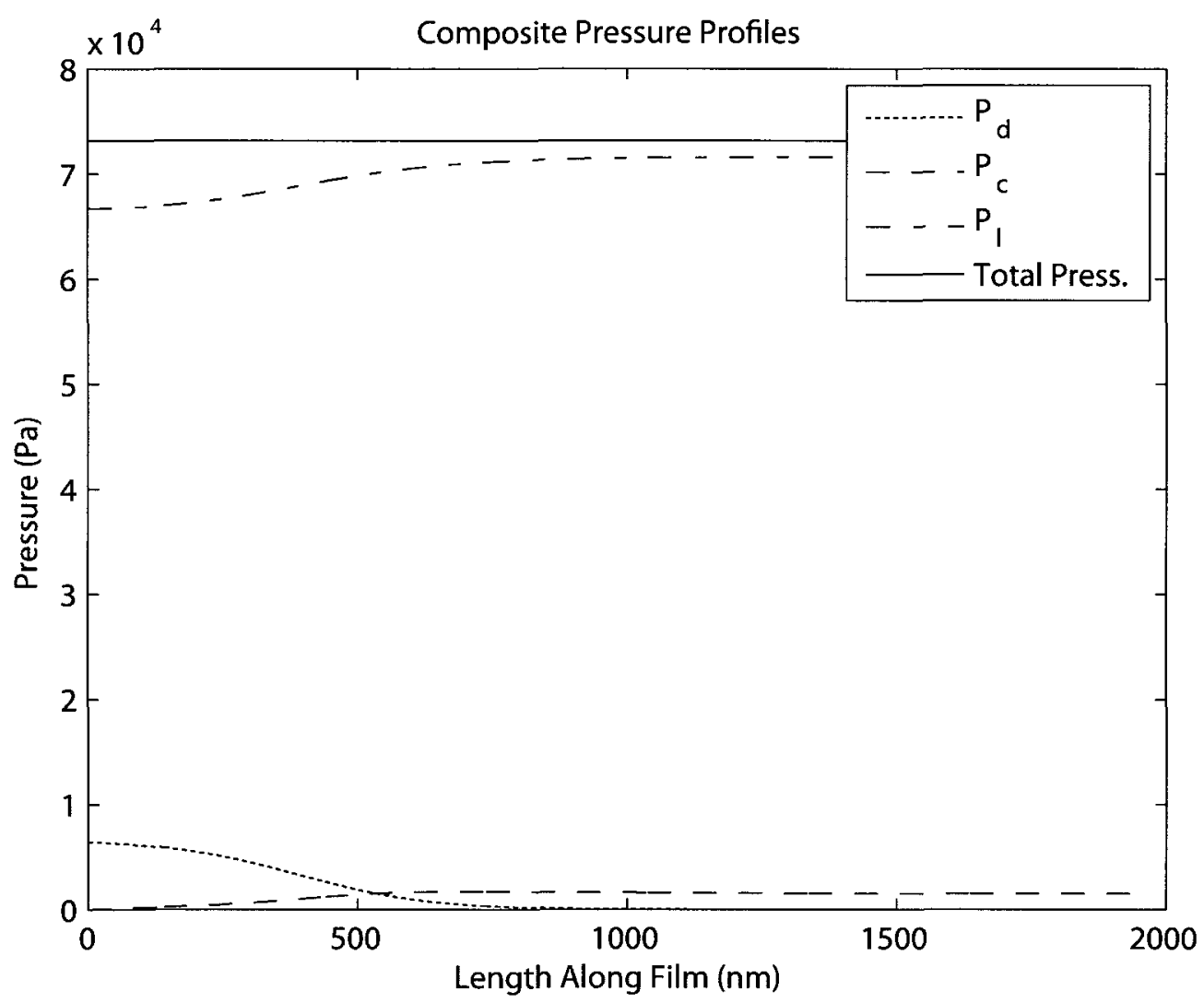

Figure 14: Composite pressure profiles

Putting all of the pressures together, we get a composite pressure plot as shown in Figure 14. The composite pressure plot was generated so as to verify the pressure balance with the condition of a constant vapour pressure. Thus, Figure 14 confirms that the pressures sum to a constant value equal to the vapour pressure. 


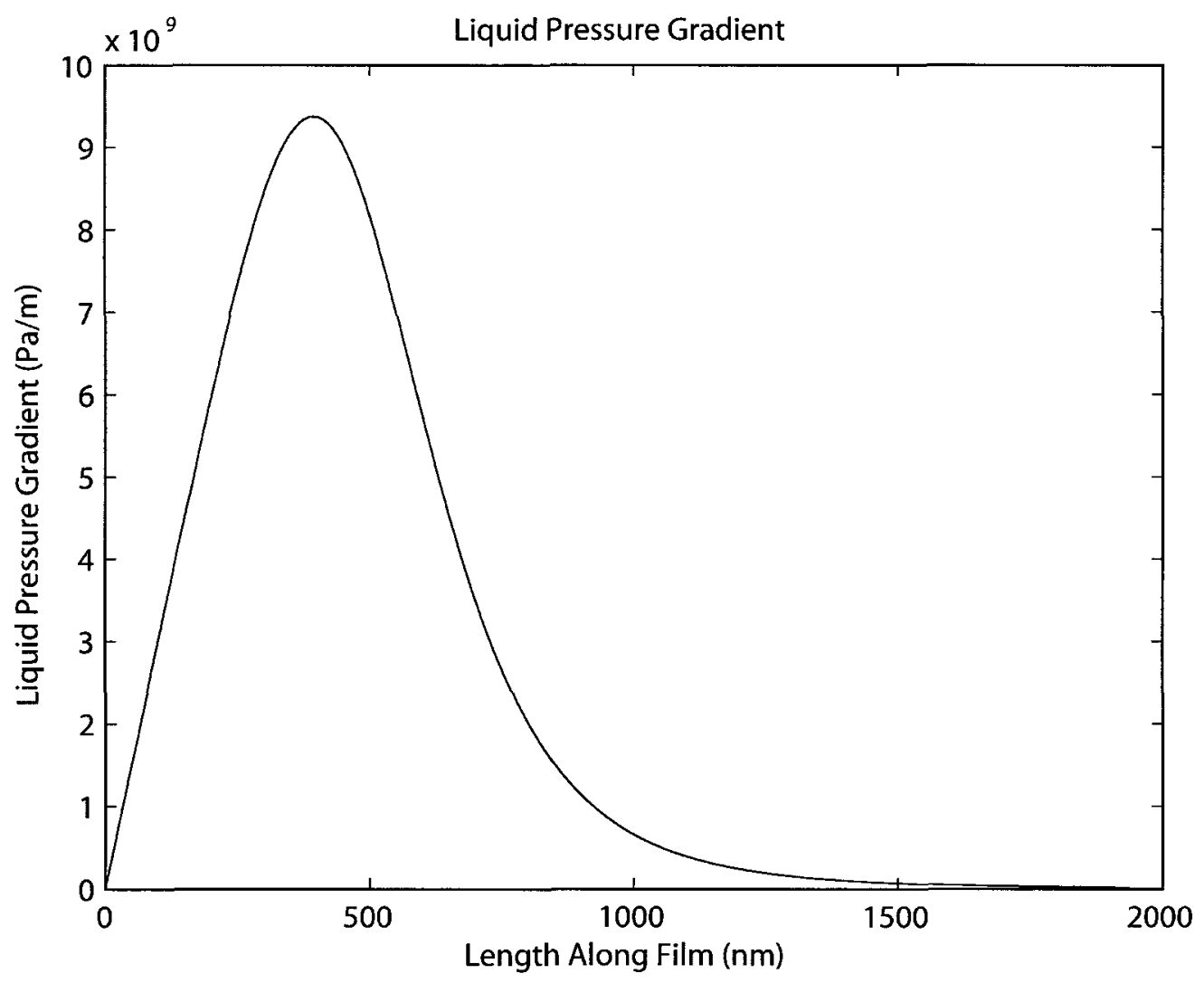

Figure 15: Liquid pressure gradient

Lastly is the liquid pressure gradient. This term was determined using the integration iteration. The location of the liquid pressure gradient peak precedes the evaporative mass flux peak, as confirmed by Wee et al. [9]. The peak magnitude as compared to that of Wee et al. [9], is approximately three times greater, yet with a film length of one third. Thus the compared values for the liquid pressure gradient as described by the integrand of Equation (33), suggests that the area under the curves is equal. This is further verified by the liquid pressure profile comparison previously discussed. 


\subsection{Uniqueness study}

With the numerical model working as desired, an investigation into the uniqueness of the solution was required. Thus formulating the question: for a given set of initial conditions, can a solution be found? To this end, different sets of initial conditions and associated perturbations were collected in Table 5. These conditions were all run using the same inputs as used previously for the basic pentane solution, with the modifications coming only from the values tabulated. The values of $\epsilon_{2}$ are the solved values for the given case, and are rounded off for brevity.

Table 5: Perturbations for uniqueness test

\begin{tabular}{|c|c|c|c|c|}
\hline Test case & $\epsilon_{1}$ & $\epsilon_{2}$ & $\epsilon_{3}$ & $\epsilon_{4}$ \\
\hline 1 & $4.2 \times 10^{-10}$ & $9.9944 \times 10^{-4}$ & $1 \times 10^{-3}$ & 0 \\
2 & $3.0 \times 10^{-11}$ & $5.9946 \times 10^{-5}$ & $1 \times 10^{-5}$ & 0 \\
3 & $9.0 \times 10^{-12}$ & $1.7827 \times 10^{-5}$ & $1 \times 10^{-5}$ & 0 \\
4 & $7.0 \times 10^{-12}$ & $1.3848 \times 10^{-5}$ & $1 \times 10^{-5}$ & 0 \\
5 & $5.0 \times 10^{-12}$ & $9.9997 \times 10^{-6}$ & $1 \times 10^{-5}$ & 0 \\
6 & $3.0 \times 10^{-12}$ & $5.9879 \times 10^{-6}$ & $1 \times 10^{-5}$ & 0 \\
7 & $1.0 \times 10^{-12}$ & $1.9763 \times 10^{-6}$ & $1 \times 10^{-5}$ & 0 \\
8 & $9.0 \times 10^{-13}$ & $1.9244 \times 10^{-6}$ & $1 \times 10^{-5}$ & 0 \\
9 & $1.0 \times 10^{-15}$ & $2.5888 \times 10^{-7}$ & $1 \times 10^{-7}$ & 0 \\
\hline
\end{tabular}


As Table 5 indicates, for a given set of initial conditions and perturbations, different solutions can be obtained. The trends regarding the thin film length and associated characteristics are given in Figures 16 through 19. The direction indicated by the arrow within each figure, indicates the direction of decreasing $\epsilon_{1}$.

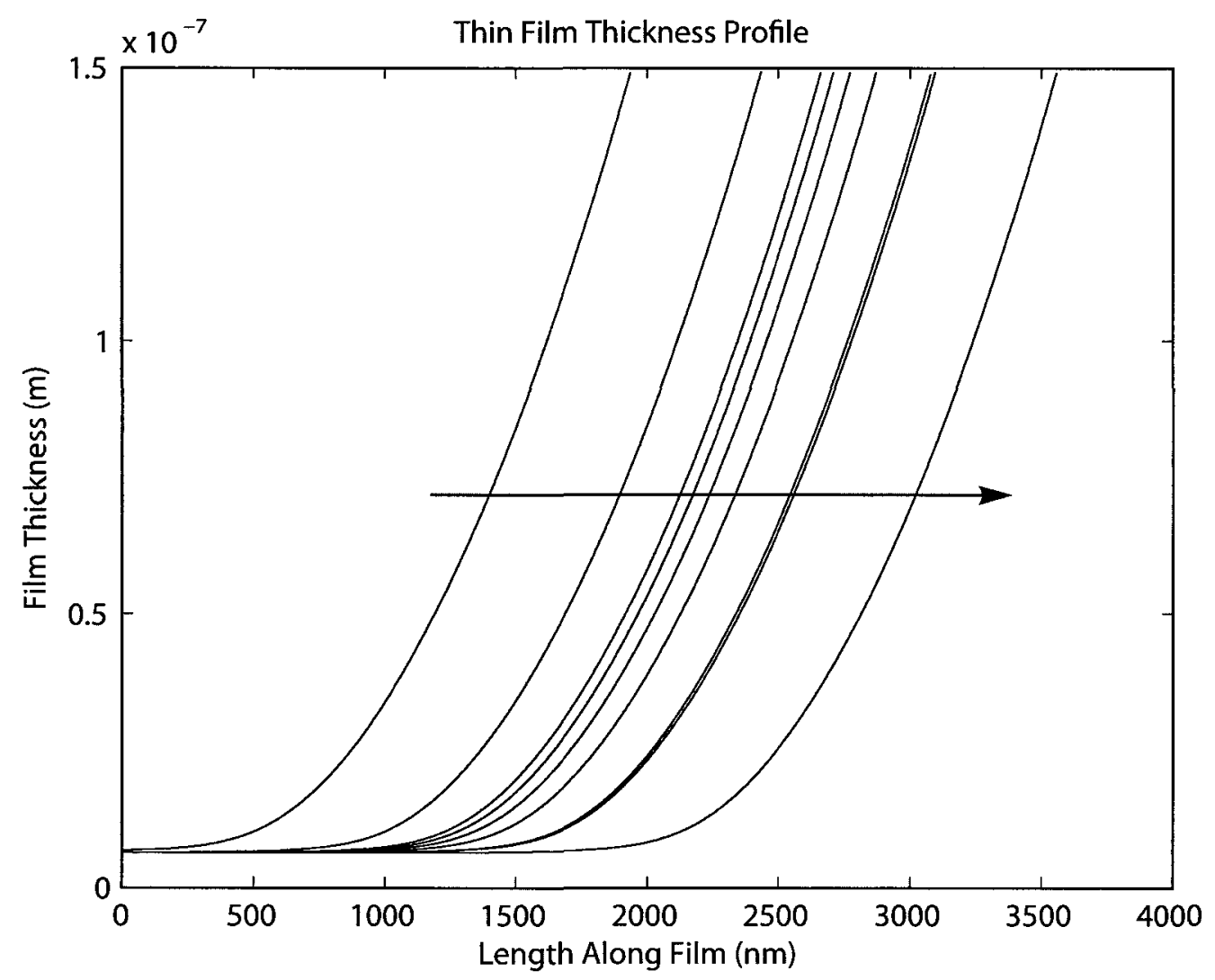

Figure 16: Thin film thickness profiles

Figure 16 illustrates the results due to variations in the perturbations applied. Thus, it can be inferred that a decrease in the perturbation applied to $\delta_{0}$, results in a longer thin film length. Of interest is the shape and consistency of the thin film thickness profile. With the decreasing perturbation size, the profile appears to shift along the length, with no significant change in shape. 


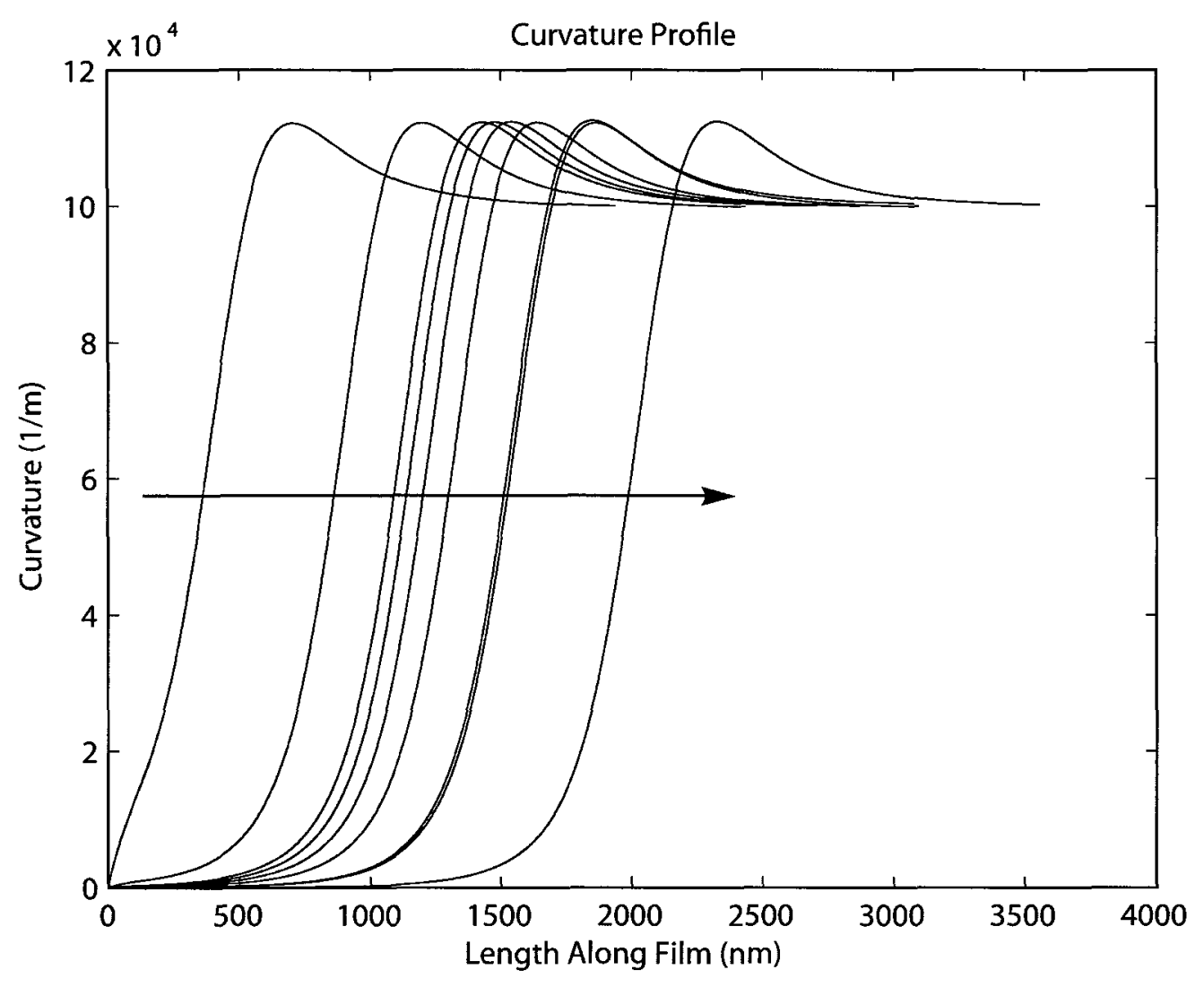

Figure 17: Local curvature profiles

To confirm the validity of the solutions, the curvature in the far field is needed. Figure 17 illustrates the curvature profile for each of the changes in perturbations. Thus it is possible to see the curvature in the far field has achieved a near constant value of $1 / r$. With the solutions confirmed via curvature, and the film thickness monotonically increasing, the heat transfer characteristics of the thin film solutions can be investigated. Note, capillary pressure is a function of curvature and surface tension, thus giving it a trend similar to that of the curvature profiles. 


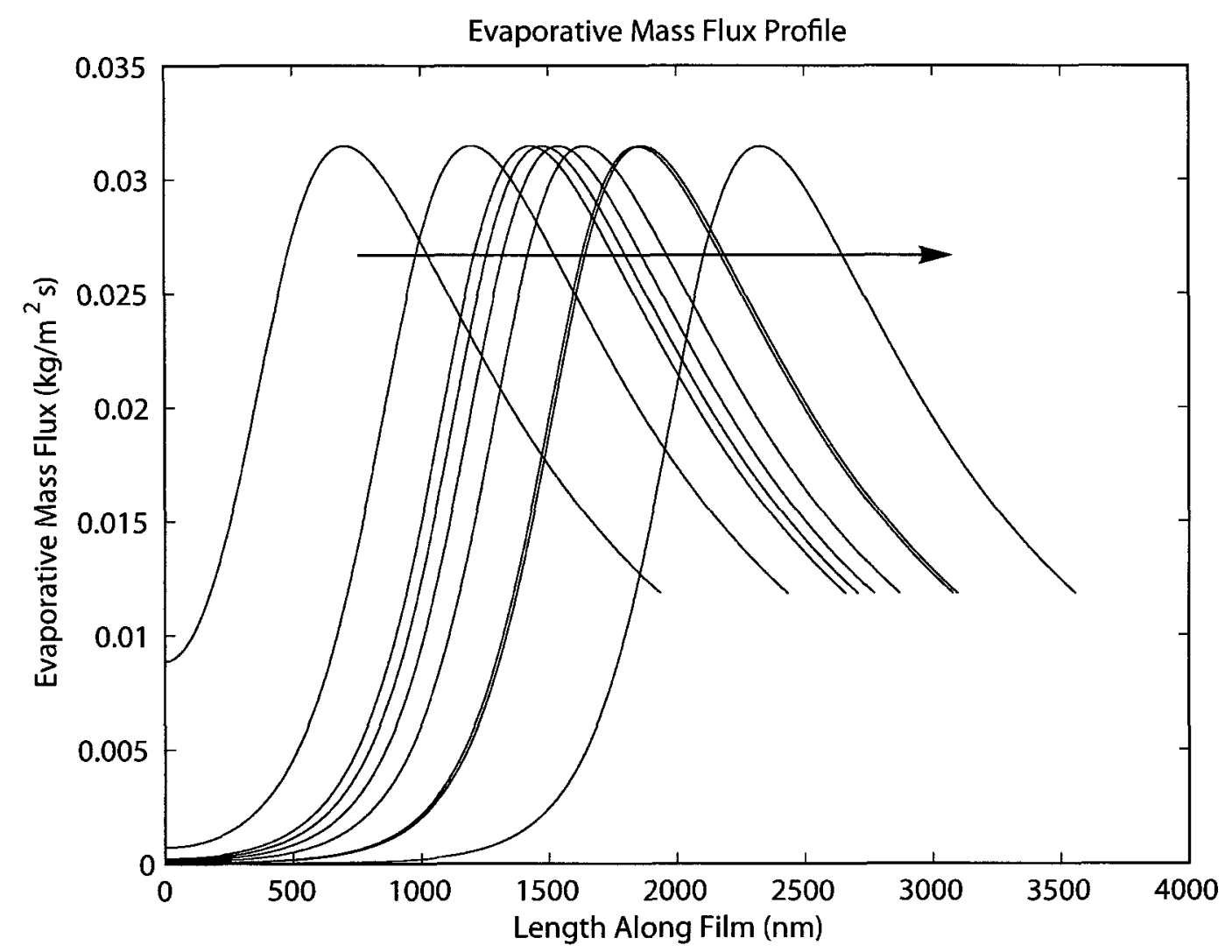

Figure 18: Evaporative mass flux profiles

The evaporative mass flux profiles, as provided in Figure 18, reach the same maximum value as previously determined. The variation in perturbation applied to $\delta_{0}$ results in a shift along the length of the thin film as seen previously. This indicates the the evaporative mass flux is the similar with a slightly longer distribution. With this shift towards a longer thin film region, the evaporative mass flux no longer has a mass flux jump at the start of the thin film. This confirms the fact that a smaller perturbation applied to $\delta_{0}$ is more consistent with our assumptions.

This trend in shifting the thin film length is observed in all cases of $T_{l v}, d P_{l} / d x, P_{l}$ and $P_{d}$. The relationship between the solved value of $\delta^{\prime}$ and the perturbation applied to $\delta_{0}$ is plotted in Figure 19. 


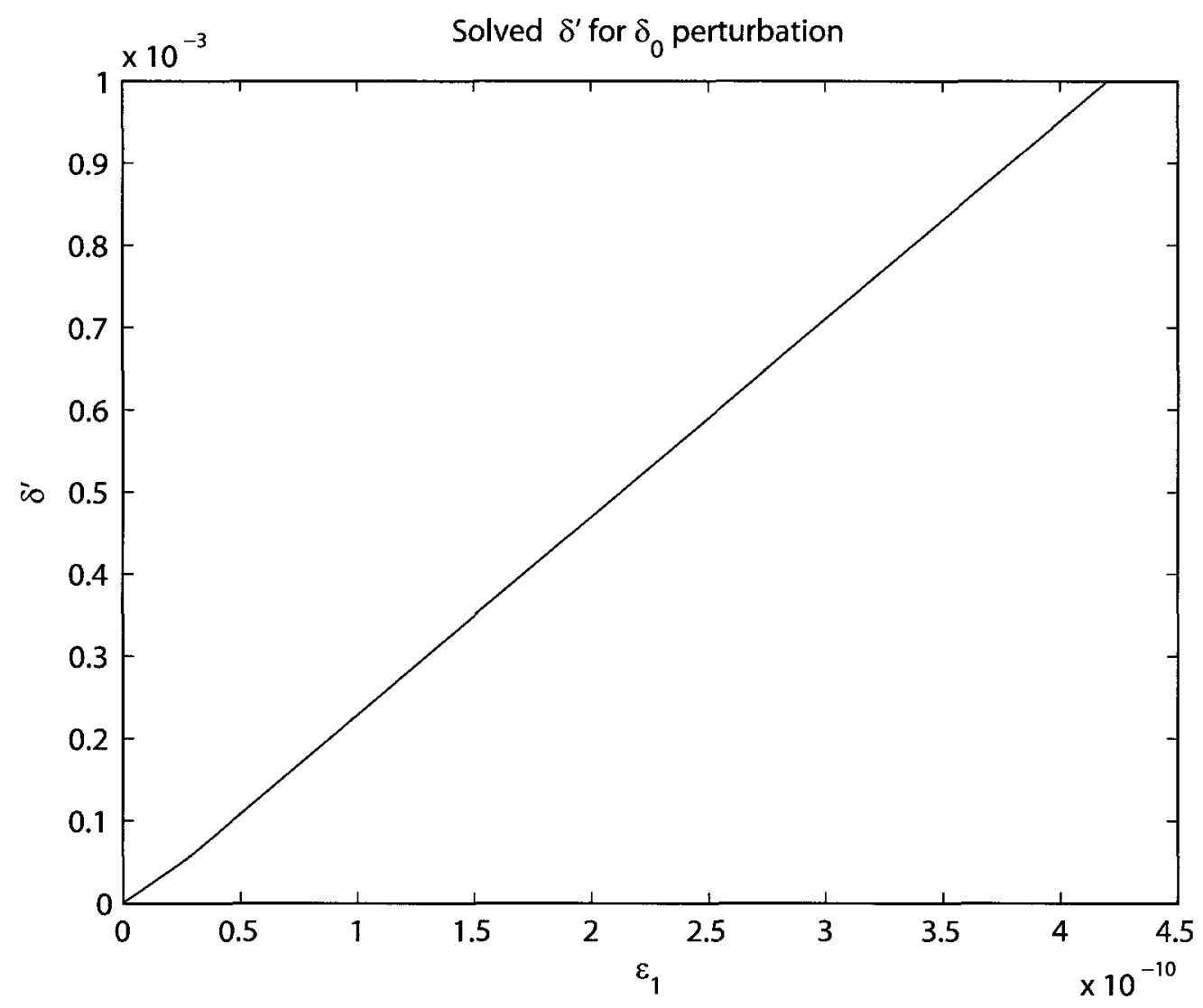

Figure 19: Relationship between $\epsilon_{1}$ and $\delta^{\prime}$

As shown in Figure 19, the relationship between the solved value of $\delta^{\prime}$ and the perturbation $\epsilon_{1}$, is a linear trend with a fit function of $\delta^{\prime}=2.3843 \times 10^{6} \epsilon_{1}$. This trend fit confirms that the solution nearest the zero perturbation is that of the trivial solution.

Thus, the solution to the thin film problem is not unique, and can have an effect on the thin film profile. Although the perturbations applied result in the shifting of the thin film length, it can also result in the unexpected jump as seen in Figure 18 , for the case of $\epsilon_{1}=4.2 \times 10^{-10}$. Considering the effects of perturbation size on thin film length and profile, the perturbations need to be small as compared with the value they are perturbing. 


\subsection{Parametric study}

With the development of the code, it has been proven that a solution for the third order differential equation can be obtained. The solution to Equation (39), is not unique, but rather a function of the applied perturbation $\epsilon_{1}$ and the required perturbation $\epsilon_{2}=\delta^{\prime}$. The parametric study is meant to ensure perturbations applied in the numerical model are of the correct order of magnitude. Thus, the objective of this parametric study, was to determine how the solution to Equation (39) was affected by the applied perturbations.

For the parametric study, the conditions from the basic pentane solution were used. The code was permitted to run until either a constant curvature was achieved or the curvature became negative. The perturbations were varied independently of each other, and were kept within the range for which a valid solution had been obtained.

The results of the numerical simulations were plotted in a three dimensional format with the axis covering the perturbation range, thin film length and the desired characteristic, typically curvature. The three dimensional plots are provided below in a two dimensional format, with directional arrows to indicate the direction of increase in the perturbation size. For cases where the code terminated early due to a negative curvature, a simple three dimensional perspective is given to show the transition more effectively. 


\subsubsection{Thickness perturbation}

The first variance is done for the adsorbed film thickness perturbation $\left(\epsilon_{1}\right)$. To determine how sensitive the differential equation is to film thickness perturbations, a set of twenty evenly spaced points provided the range of $\epsilon_{1}$. The range is based on a percentage system, using the adsorbed thickness as the reference for the percentage range. Thus the perturbations range from $0.01 \%$ to $0.2 \%$ of $\delta_{0}$.

The variance of $\epsilon_{1}$ as a percentage of $\delta_{0}$, resulted in some premature terminations. These cases are a result of the curvature becoming negative, and are given below in Figure 20 .

\section{Curvature Profile}

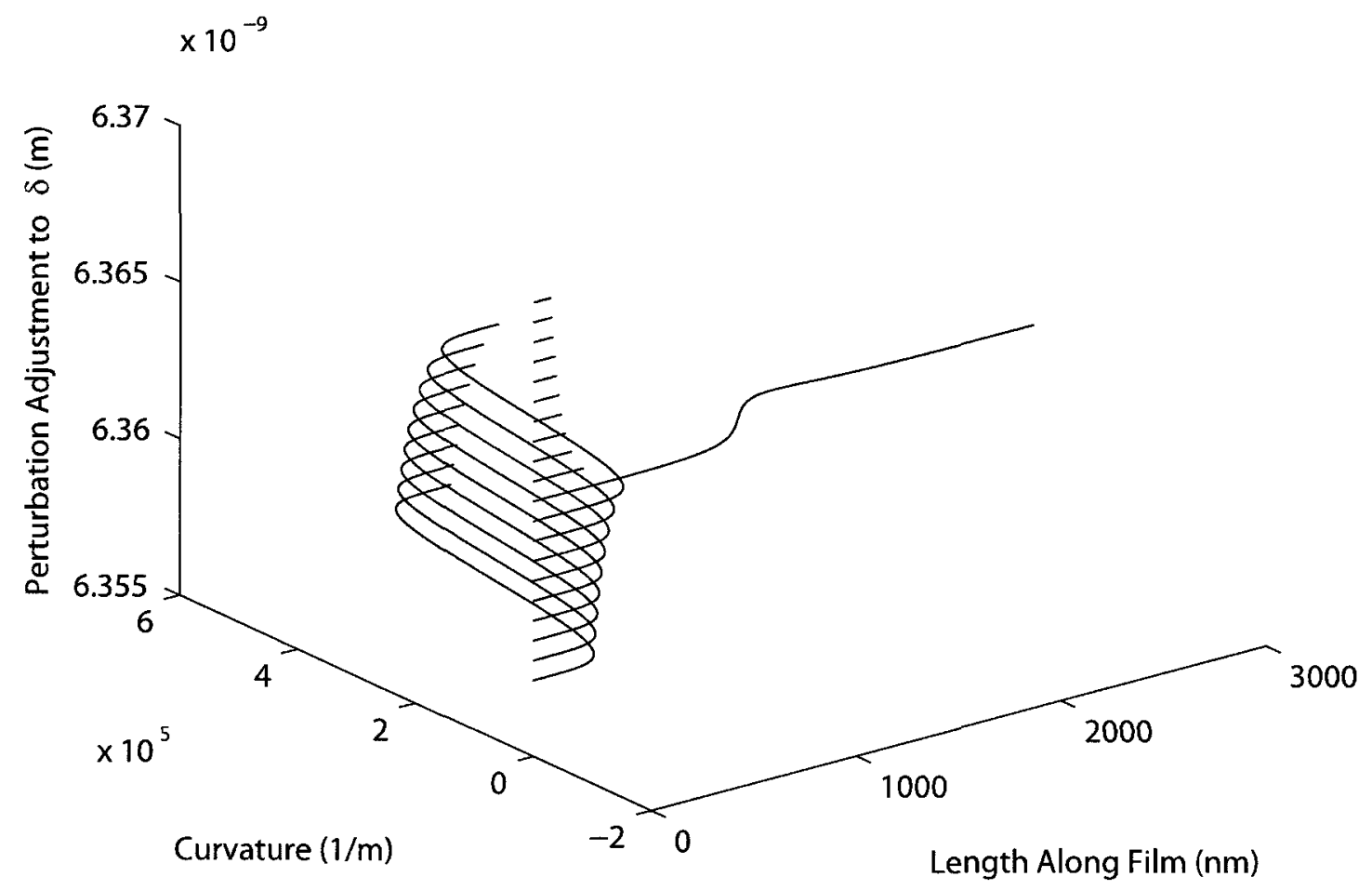

Figure 20: Curvature variance with $\epsilon_{1}$ 
The three dimensional plot shows how the curvature is sensitive to the perturbation of $\epsilon_{1}$. During the running of the numerical simulations it was observed that the curvature of the far field had a tendency to be stable within certain regions. This stability is seen in Figure 20, as the variance of the thickness perturbation has only a small effect on the final curvature for $\epsilon_{1} \leq 0.1 \%$. Outside of this, the second stable region resulted in that of a negative constant curvature grouping. For the specific case of a Young-Laplace solution, the solution was in the transition zone where the curvature underwent a significant shift in far field curvature.

Given the multitude of premature terminations, a new bracket for the search was selected. The refined bracket covers $0.05 \% \leq \epsilon_{1} \leq 0.15 \%$, with twenty evenly space data points. The results still had negative curvature returns, but were still used in the following figures. Note that the conditions which give rise to the Young-Laplace solution fell between the values used in the perturbation vector. This indicates that the switch in curvature occurs in a difference less than $0.005 \%$ of $\delta_{0}$, and hence the extreme sensitivity. 


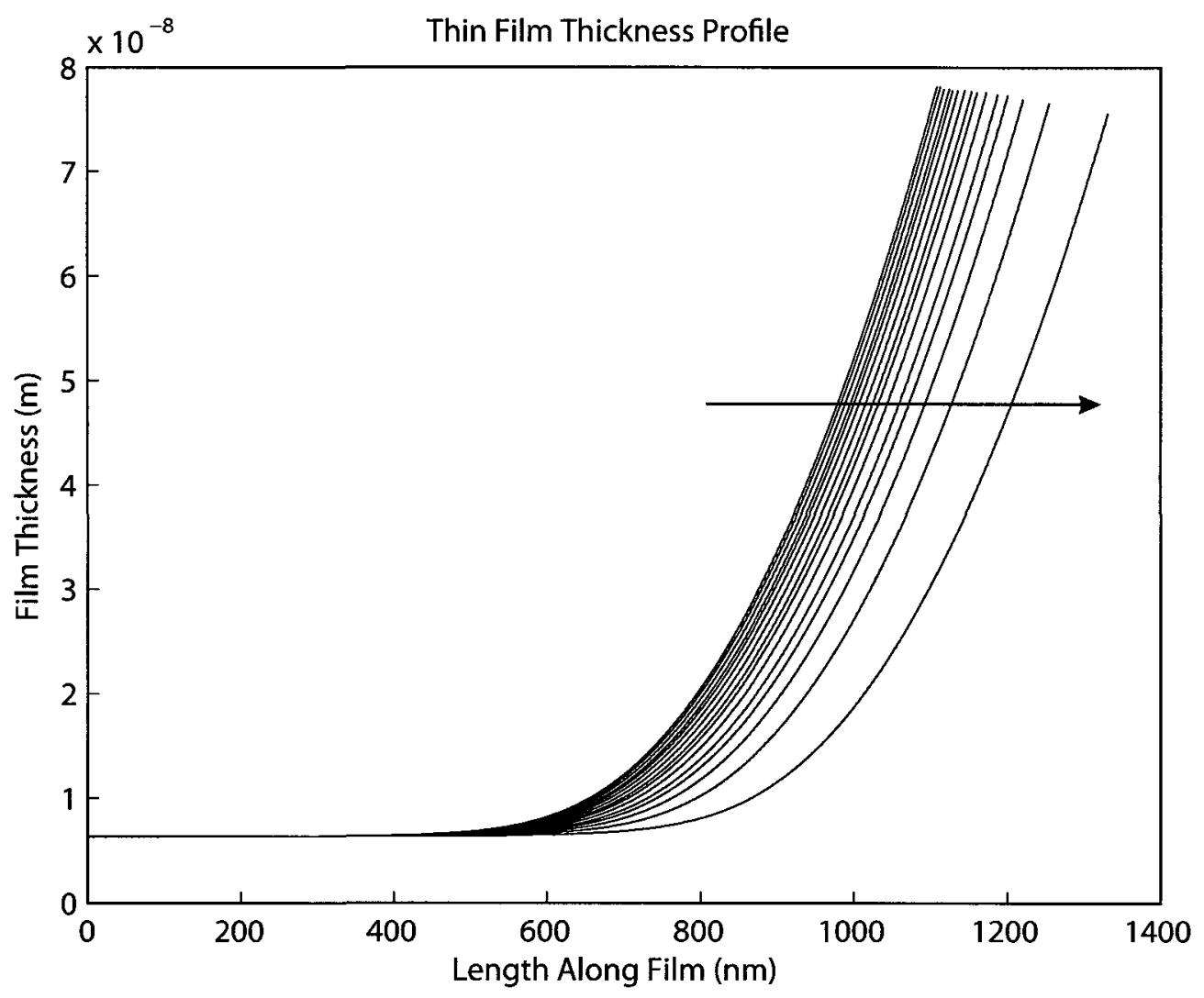

Figure 21: Thin film thickness variance with $\epsilon_{1}$

As the adsorbed film thickness perturbation increases in size, the thin film length has the tendency of increasing. This increase in thin film length and also has the effect of slightly modifying the thin film interface shape, as seen in Figure 21. This result is different than that seen previously in uniqueness study, where the interface was simply shifted along the length of the thin film region. In addition, the length of the thin film for the converged solutions is less than half that of the Young-Laplace solution. 


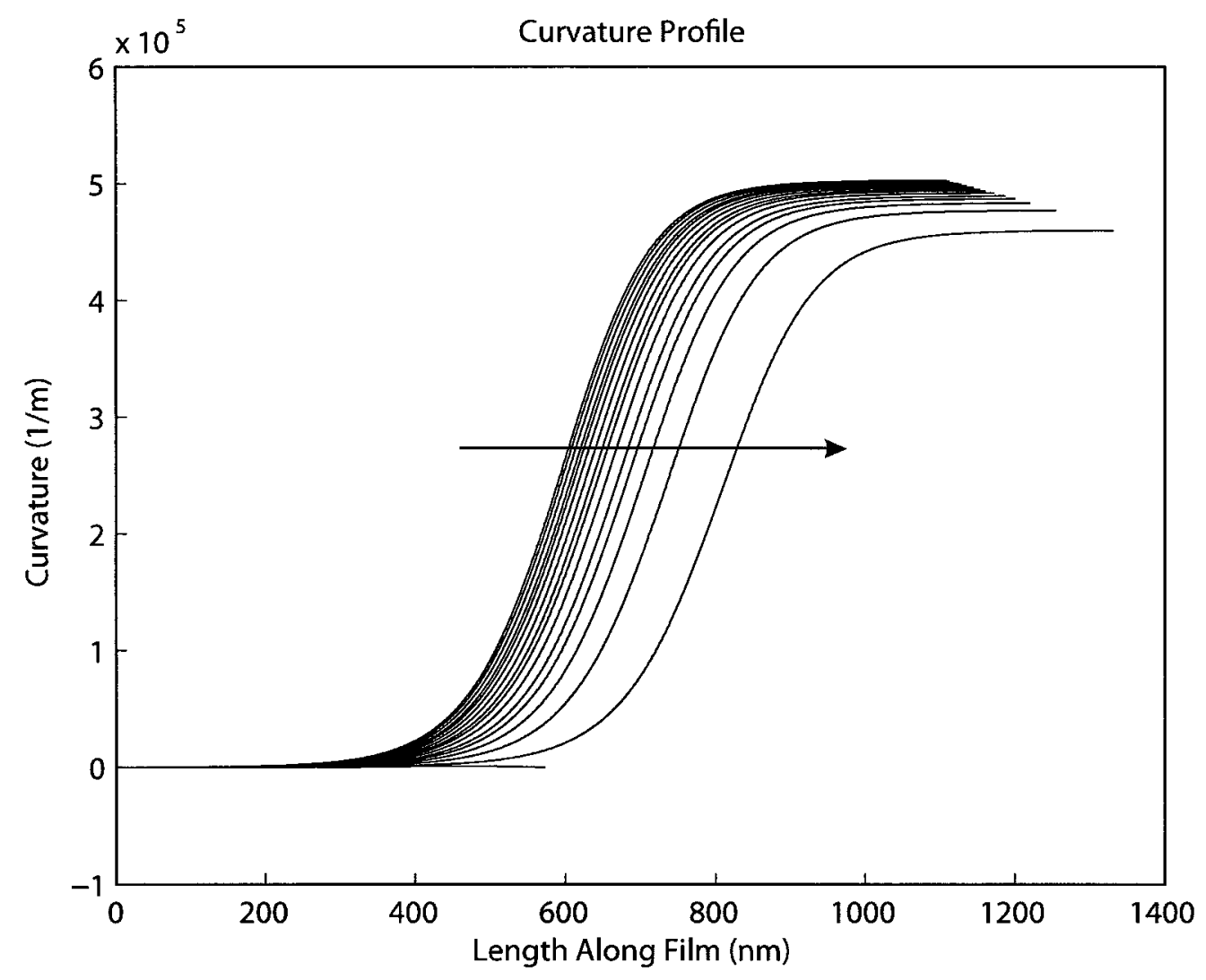

Figure 22: Curvature variance with $\epsilon_{1}$

The curvature variance as shown in Figure 22, illustrates how the curvature has a tendency to be stable within a specified region. For the this specific case, the curvature was stable for $5 \times 10^{5} \leq \kappa \leq 4.5 \times 10^{5}$, after which a swift transition occurs and the curvature is apparently stable as a negative value.

The stability regions of the governing third order differential equation were an unexpected result of the parametric study. The reasons behind the stability are unknown and warrant future investigation, as it may provide insight into the far field curvature solution, which is currently taken to be the Young-Laplace solution. 


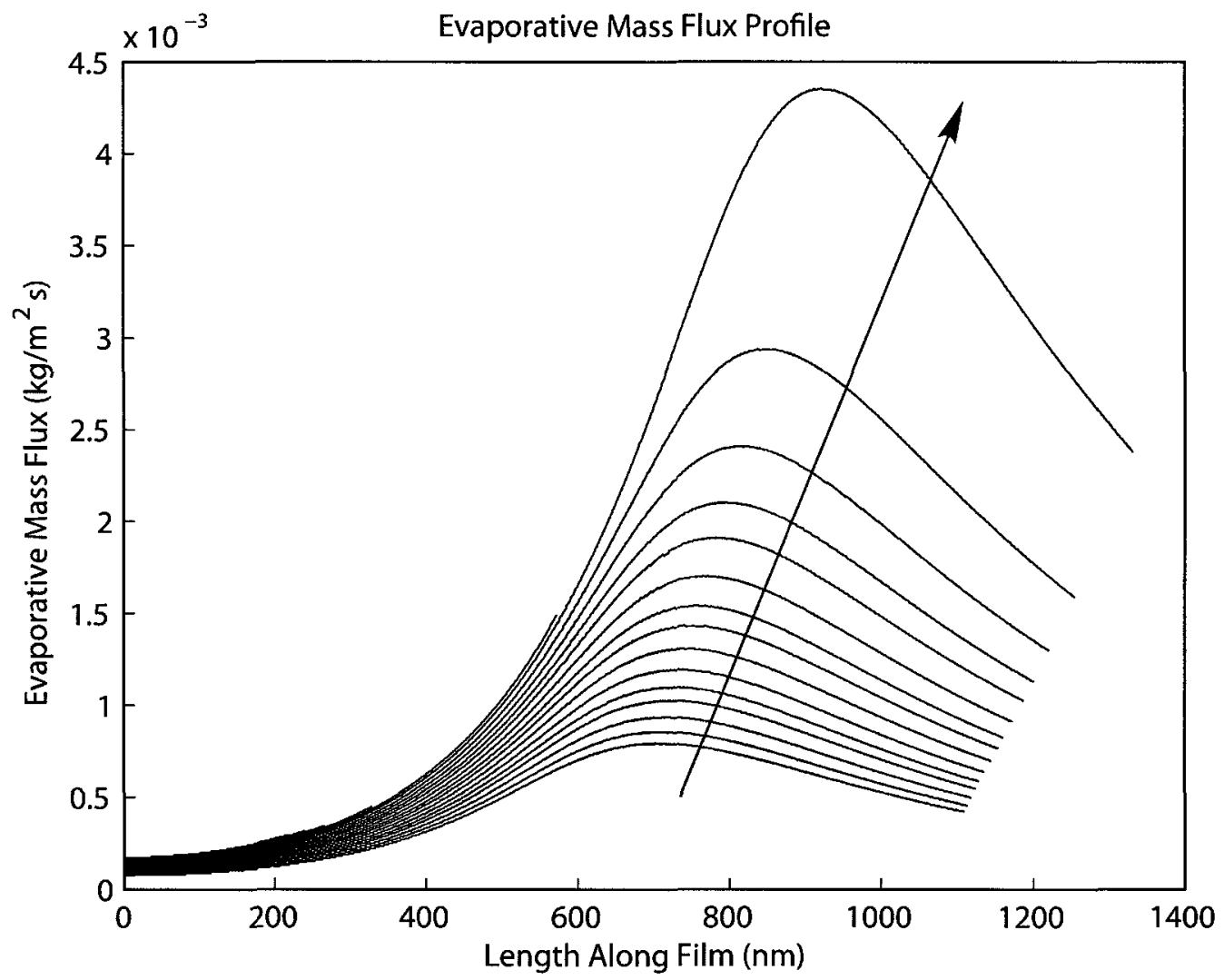

Figure 23: Mass flux variance with $\epsilon_{1}$

For the evaporation taking place in the thin film region, it is possible to see in Figure 23, that the peak magnitude and location are affected by the film thickness perturbation. The directional arrow illustrates how the increase in film thickness perturbation has the effect of substantially increasing the height of the peak as well as its breadth. 


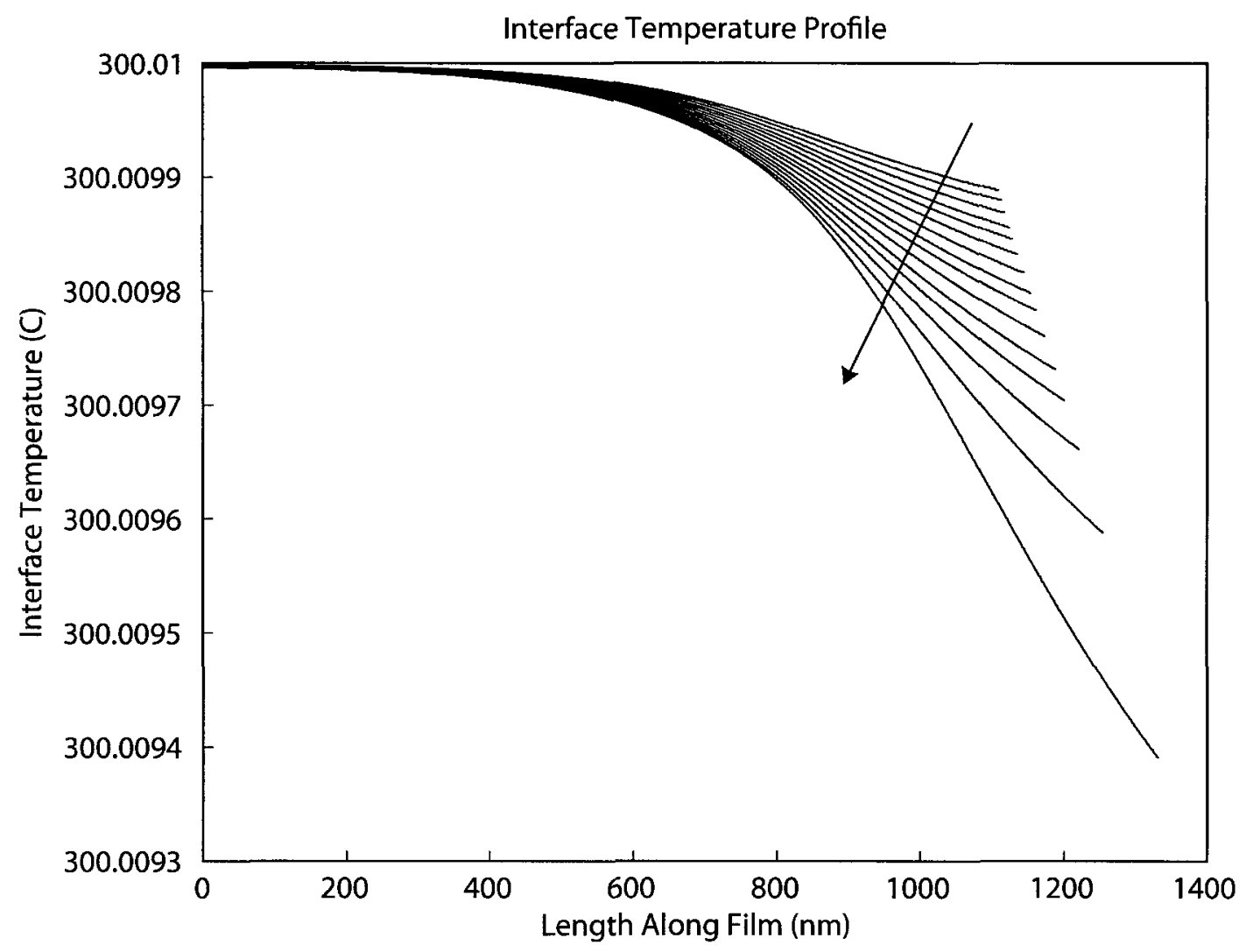

Figure 24: Interface temperature variance with $\epsilon_{1}$

The interface temperature of the thin film region, as shown in Figure 24, behaves as expected. With the lower perturbations causing lower evaporation rates in the thin film region, the evaporative cooling is not as large. This lack of surface cooling gives way to a smaller interfacial temperature gradient. 


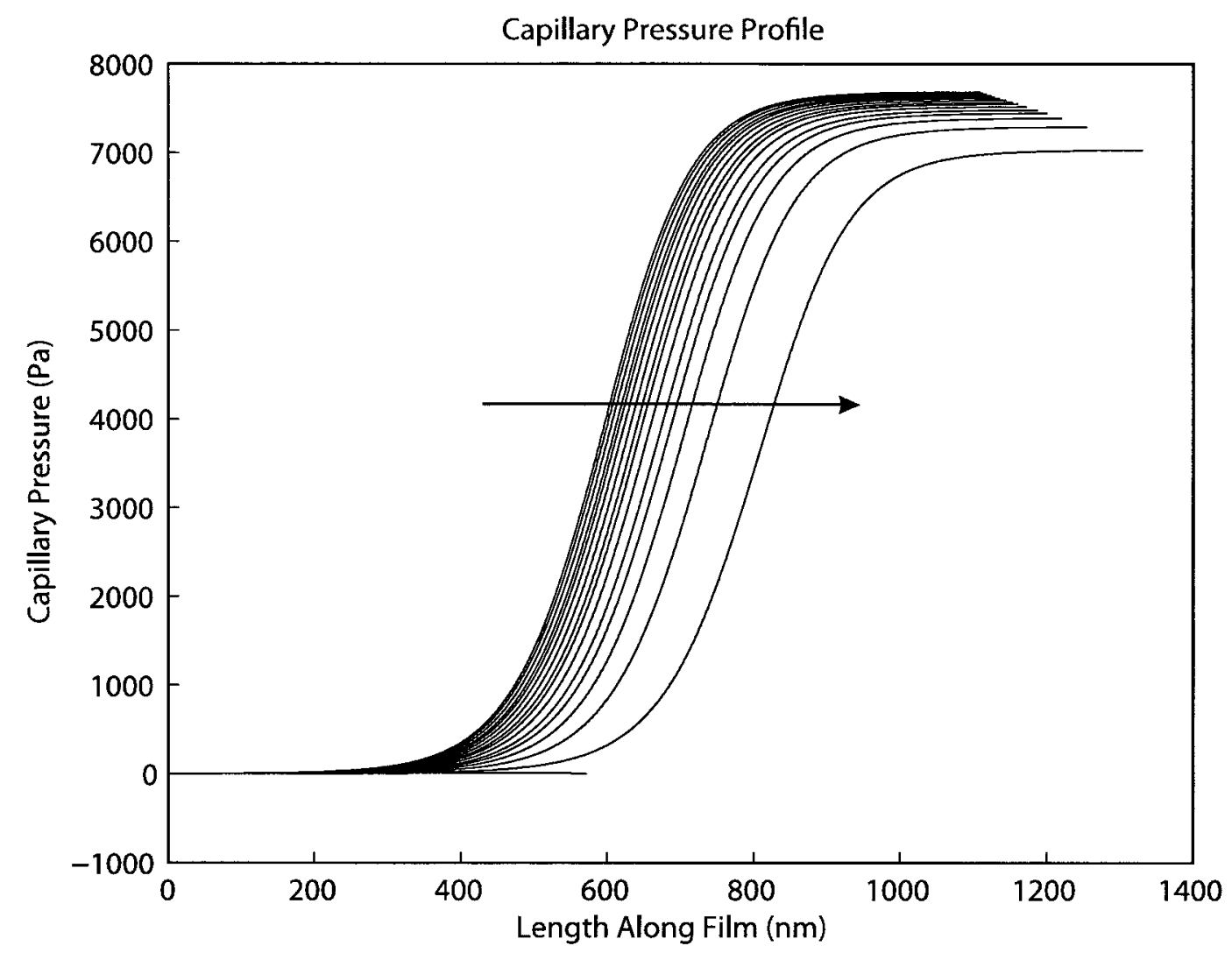

Figure 25: Capillary pressure variance with $\epsilon_{\mathbf{1}}$

The capillary pressure shift of Figure 25, is nearly identical to that of the curvature profiles shown in Figure 22. The only change is a slight progression of the capillary pressure profile as pressure gradient becomes smaller with increasing thin film length and the associated perturbation. 


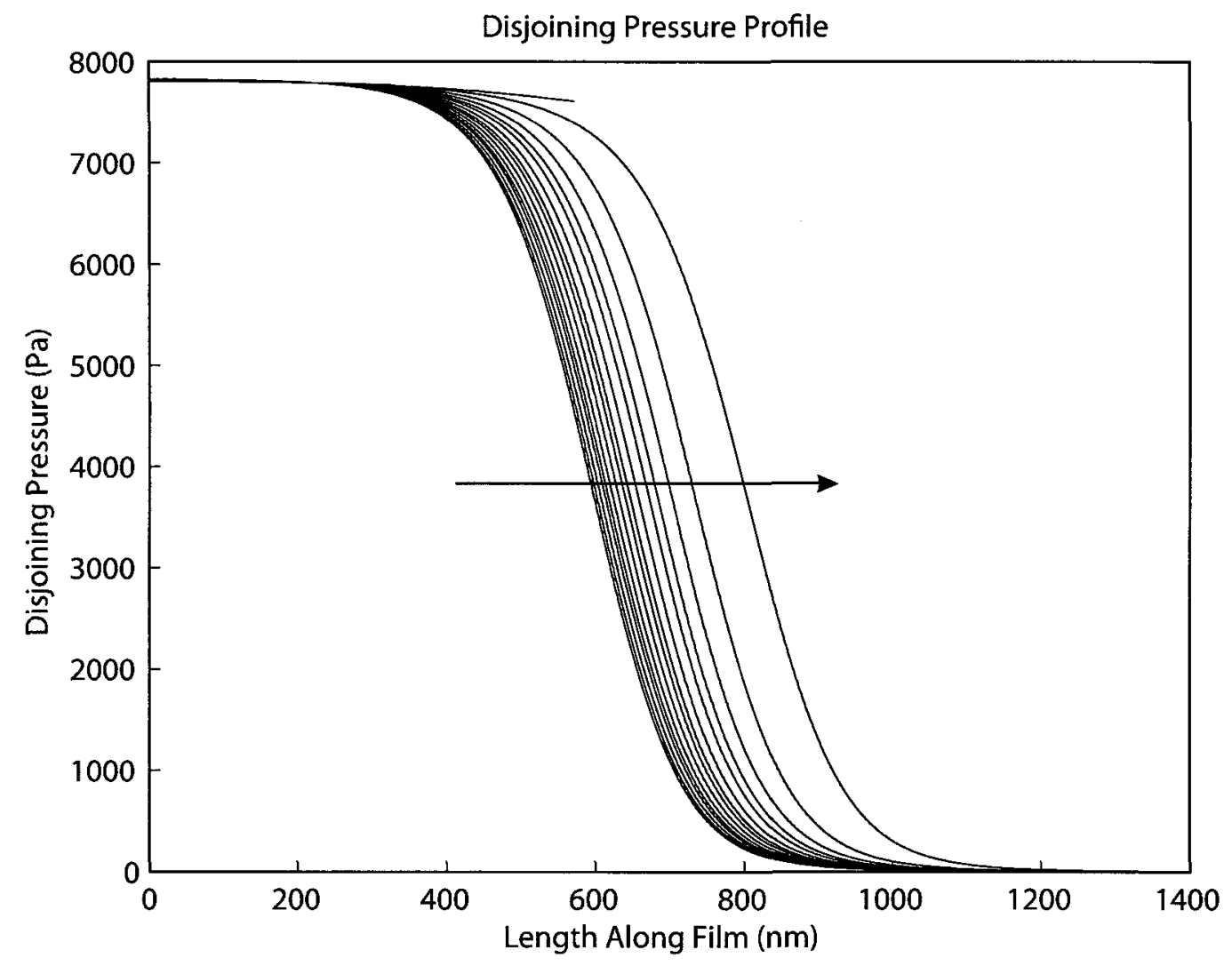

Figure 26: Disjoining pressure variance with $\epsilon_{1}$

The disjoining pressure profiles in Figure 26 exhibit the same characteristics as those previous. Much like that of the capillary pressure profile, as the thin film length increases the pressure gradient becomes less. 


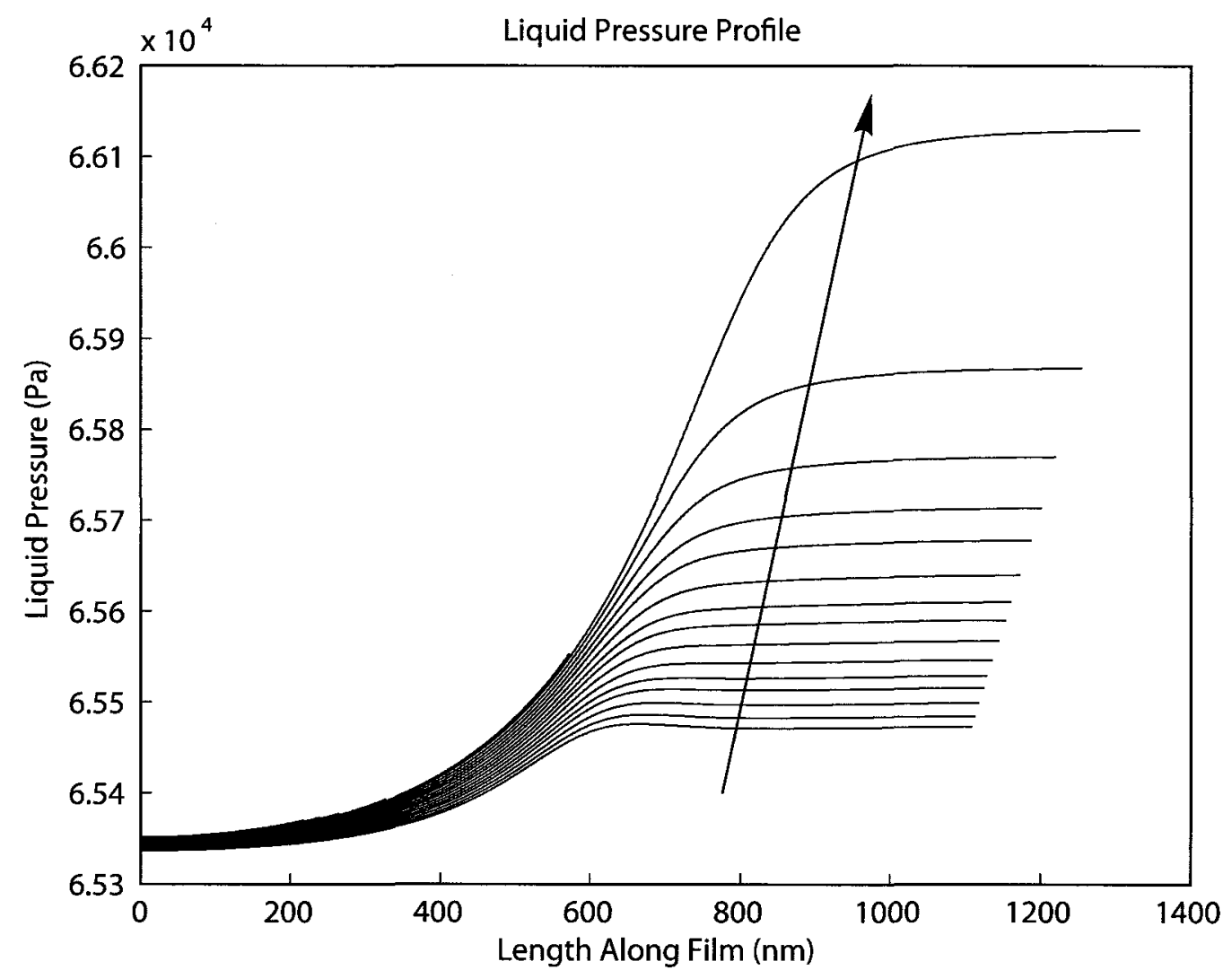

Figure 27: Liquid pressure variance with $\epsilon_{1}$

The liquid pressure profile has many of the same characteristics as that of the evaporative mass flux. As given in Figure 27, the increase in the film thickness perturbation has increased the terminal liquid pressure value. This increase can also be attributed to the changes in capillary and disjoining pressure gradients with increasing thin film length. 


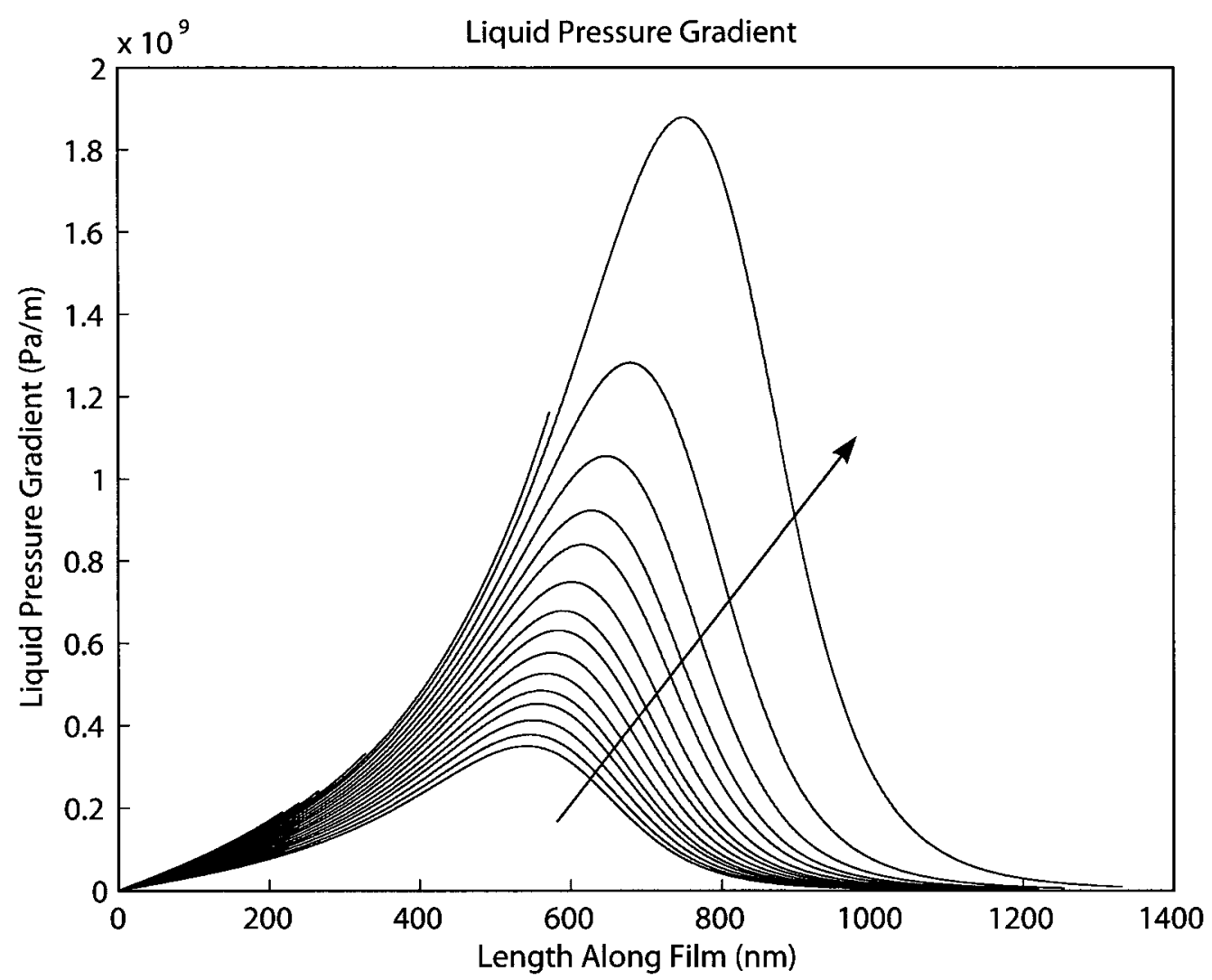

Figure 28: Liquid pressure gradient variance with $\epsilon_{1}$

Lastly is the liquid pressure gradient, as shown in Figure 28. The large changes in the liquid pressure gradient are expected to come from the changes in the disjoining and capillary pressure gradients. Provided, the validity of the cause and effect, it is possible to see how the evaporative mass flux peak occurs in concert with the liquid pressure gradient. 


\subsubsection{Slope perturbation}

The second perturbation to be investigated is for that of thin film slope. This perturbation has been used in the search algorithms of some previous numerical models with a third order differential equation $[3,9]$. It is for this reason that it has been used as the search parameter for all of the previous solutions. To ensure it is suitable as a search parameter, its sensitivity is investigated here.

For the parametric study of $\epsilon_{2}$, the values of the other perturbations were held constant. The perturbation vector consisted of twenty equally spaced points, with a spread of $1 \times 10^{-5} \leq \epsilon_{2} \leq 2 \times 10^{-5}$. The results of which are given graphically, Figure 29 , as a three dimensional plot so as to illustrate the trend in curvature shift as a function of the perturbation applied and thin film length.

\section{Curvature Profile}

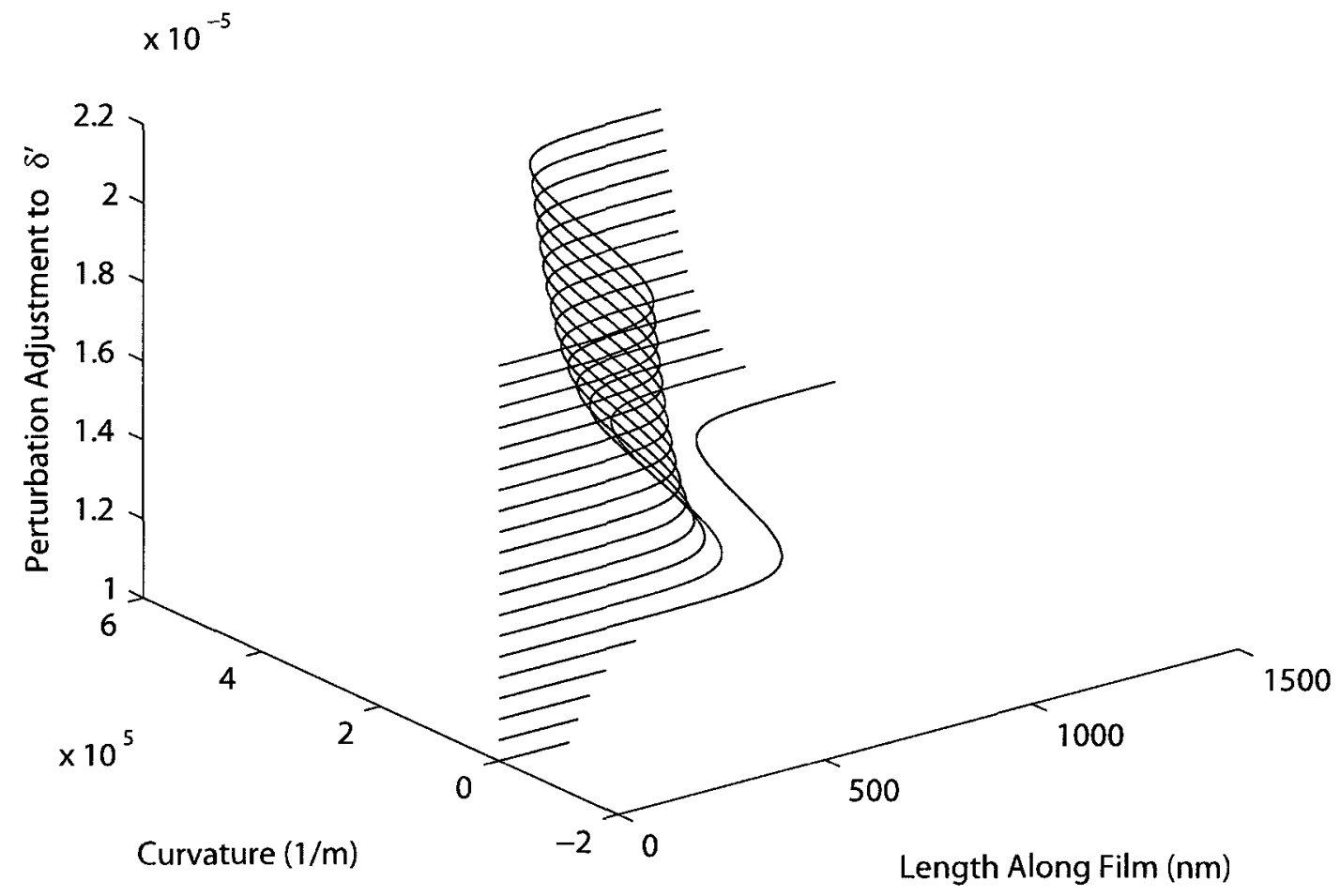

Figure 29: Curvature variance with $\epsilon_{2}$ 
As shown in Figure 29, the curvature converged to a positive value in most of the cases considered. As with the film thickness perturbation $\left(\epsilon_{1}\right)$, the curvature tended to converged in a certain region, outside of which the curvature would go to a negative value. Thus, the slope perturbation has a similar stability characteristic as that of the thickness perturbation.

Once again, the results have been put into a two dimensional format, with a directional arrow. The arrow points in the direction of increasing slope perturbation size.

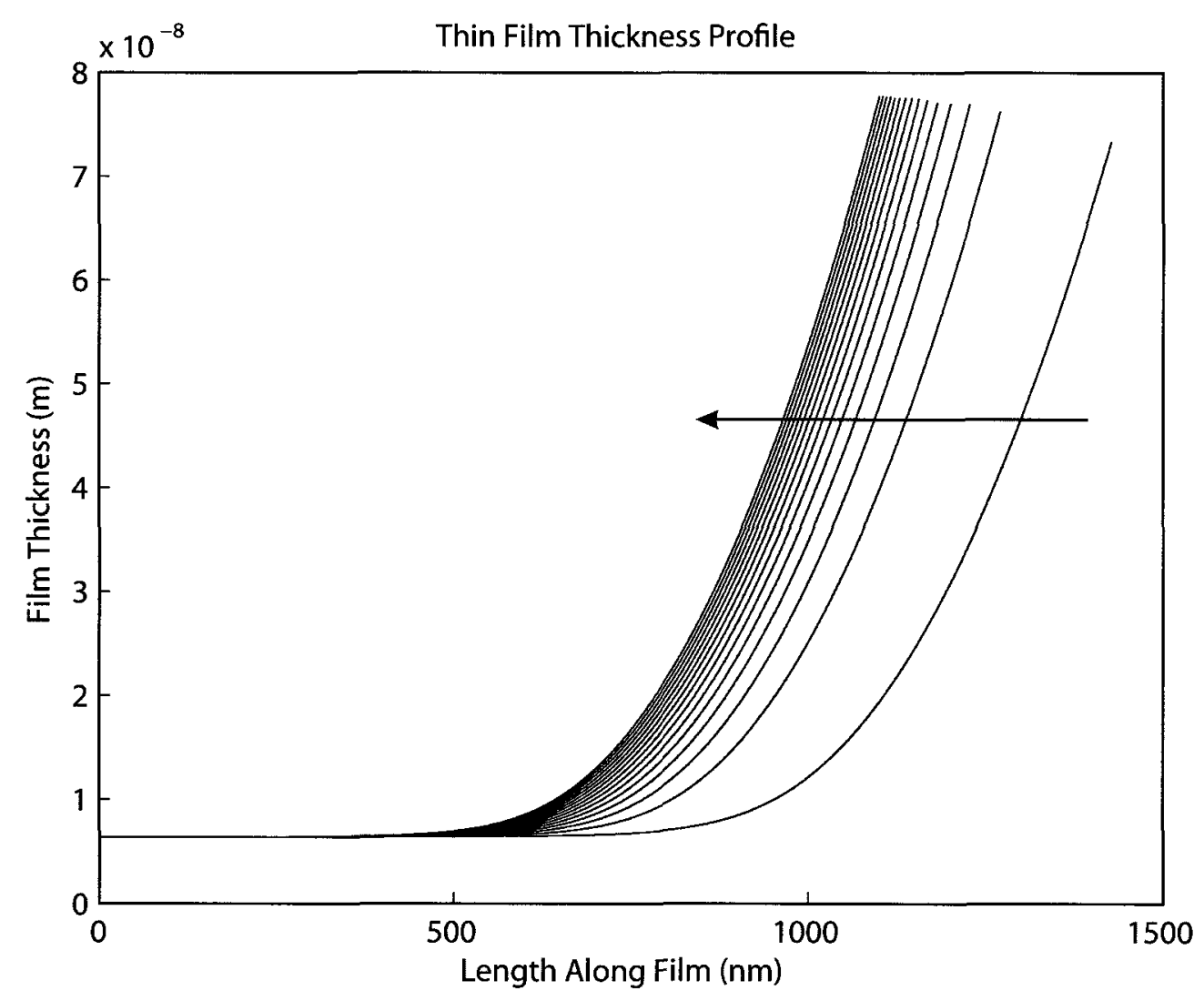

Figure 30: Film thickness variance with $\epsilon_{2}$

The film thickness profiles as given above in Figure 30, shows that the thin film surface undergoes a change in the length of the thin film. This shortening of the thin film region with increase in $\epsilon_{2}$ is opposite to that observed in the variance of $\epsilon_{1}$. 


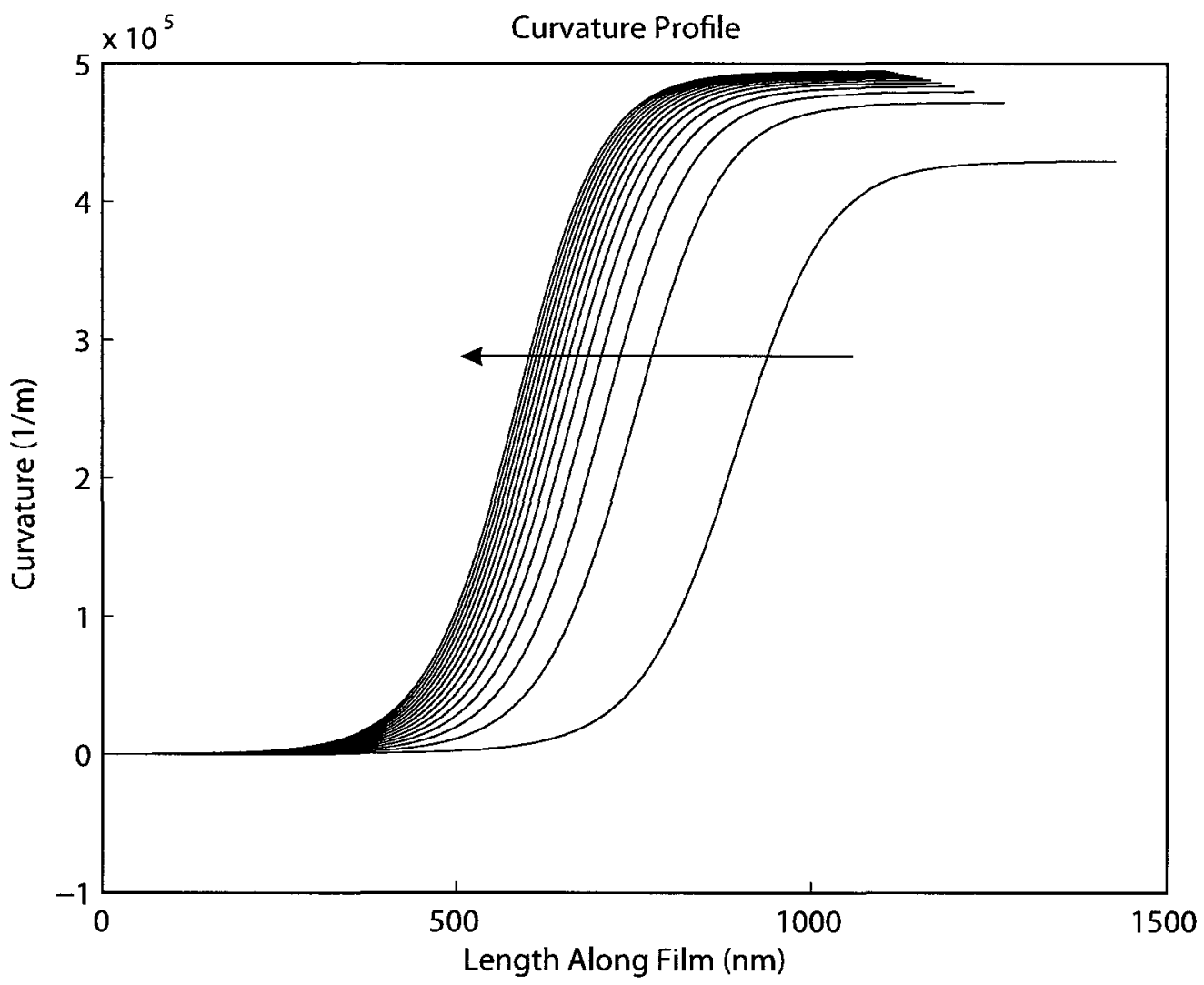

Figure 31: Curvature variance with $\epsilon_{2}$

As discussed in the review of the three dimensional curvature plot, the curvature values tend to apparent regions of stability. This is clearly shown in Figure 31 . The converged curvature values undergo a switch from a positive value, to a negative value within a change of $\Delta \epsilon_{2}=0.1 \times 10^{-5}$. Lastly, the interface shape remains near constant with only a minor change in profile. 


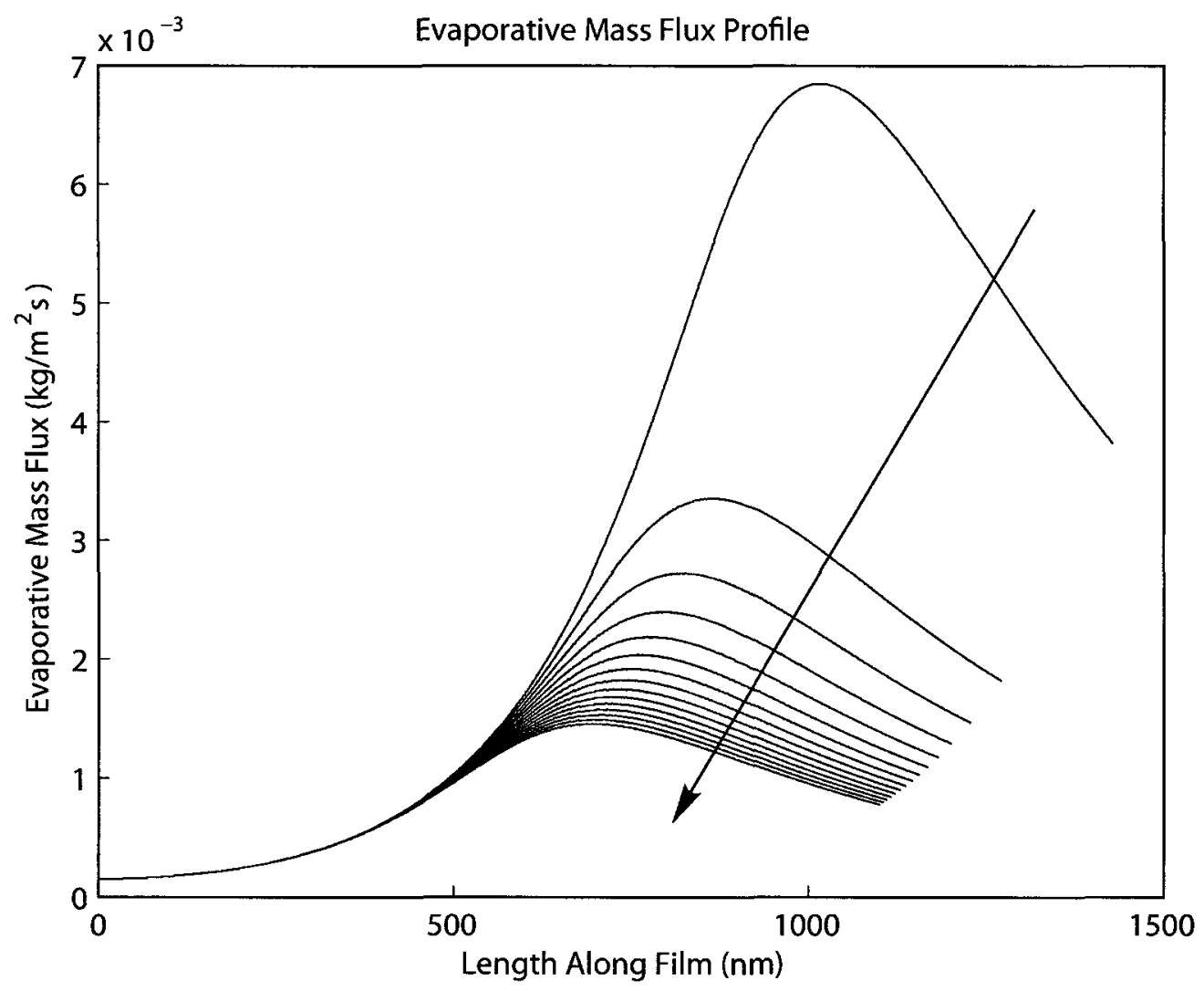

Figure 32: Evaporative mass flux variance with $\epsilon_{2}$

The evaporation rate and peak location given above in Figure 32, show a significant jump in the peak magnitude and change in profile. As such, the smaller the perturbation applied to $\delta^{\prime}$ gives way to a greater evaporative mass flux in the thin film region. This is important as the fundamental assumptions used in the adsorbed to thin film transition region, stipulate the values to be zero. 


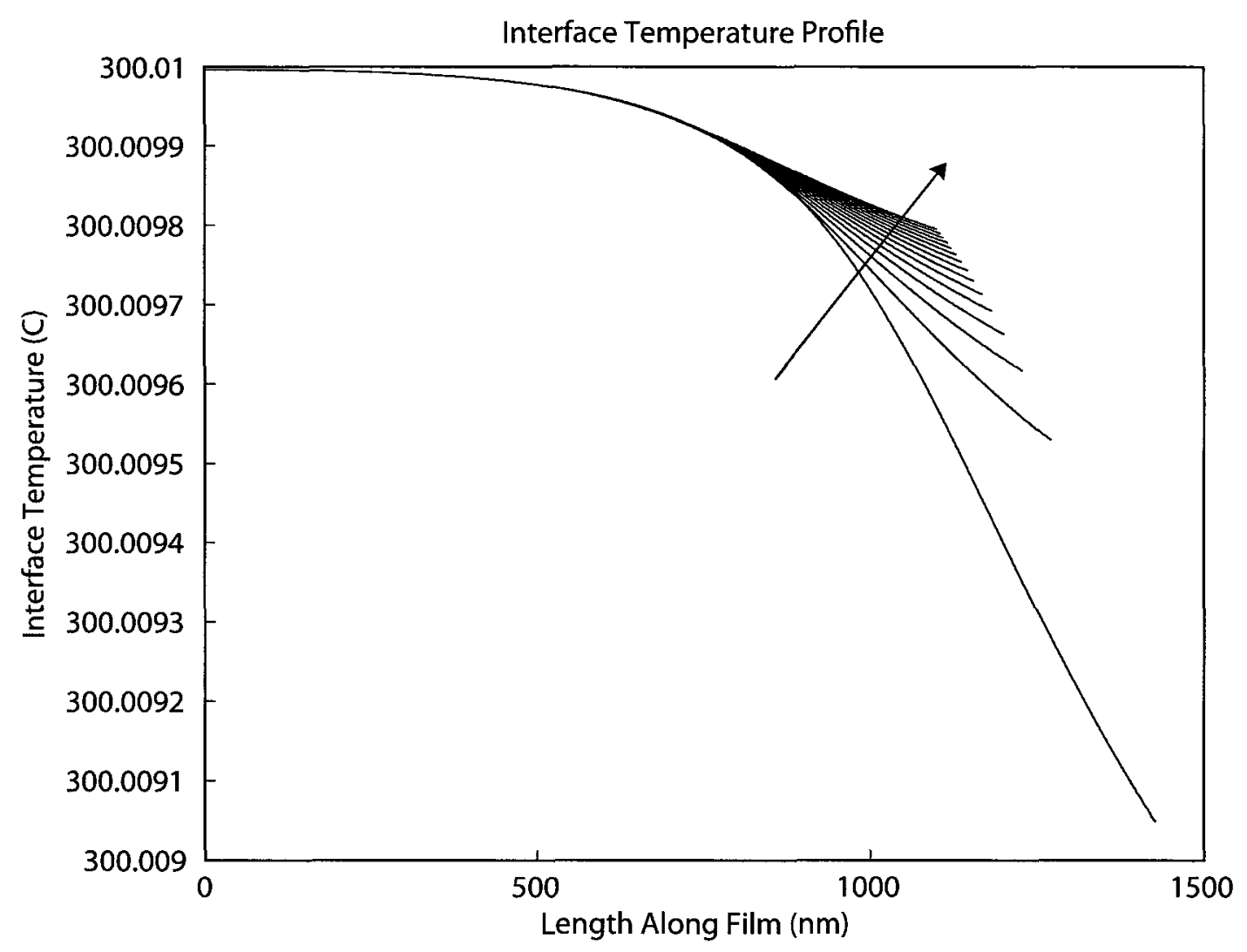

Figure 33: Interface temperature variance with $\epsilon_{2}$

The interface temperature profiles as provided in Figure 33, reiterate the points made previously. Of note is how the temperature profile shifts upward with and with an increase in the slope perturbation. Thus, the effect of the slope perturbation is opposite that of the film thickness perturbation.

The remaining thin film characteristics exhibit all of the aforementioned traits with regards to the increase in slope perturbation magnitude. The overall trend in the perturbation study of $\epsilon_{2}$, is opposite that of $\epsilon_{1}$. Thus, the parametric study of $\epsilon_{2}$ has confirmed the observations of previous works, for which the thin film slope perturbation is sensitive $[3,9]$. 
With the parametric study of both $\epsilon_{1}$ and $\epsilon_{2}$ complete, it is possible to make an observation regarding their relationship as presented earlier in Figure 19. The linear trend represented by $\delta^{\prime}=2.3843 \times 10^{6} \epsilon_{1}$, describes a confirmed set of solutions for variances in $\epsilon_{1}$ and $\epsilon_{2}$, that result in a converged far field curvature of $1 / r$. If the values selected for the different perturbations are below the $1 / r$ solution set, the thin film length will tend to increase with increasing $\epsilon_{1}$. However if the perturbations are selected to be above the $1 / r$ solution line, the thin film length will shorten with an increase in $\epsilon_{2}$.

\subsubsection{Curvature perturbation}

The last of the parametric studies was completed for the second derivative of the thin film thickness $\left(\epsilon_{3}\right)$. The perturbations applied to $\epsilon_{3}$ consisted of twenty evenly spaced points covering the range of $1 \times 10^{-7} \leq \epsilon_{3} \leq 1 \times 10^{-3}$. The selected values of the range represent the assumption that the curvature is near zero in the region nearest the adsorbed layer.

The perturbations of $\epsilon_{1}$ and $\epsilon_{2}$ were chosen to be those which ensure the lower bracket of the search coincided with a solution of $1 / r$. Unlike the previous parametric studies performed, the converged curvature values were all positive and can be accurately represented in a two dimensional plot. As such, a directional arrow is used to represent the direction of increasing $\epsilon_{3}$. 


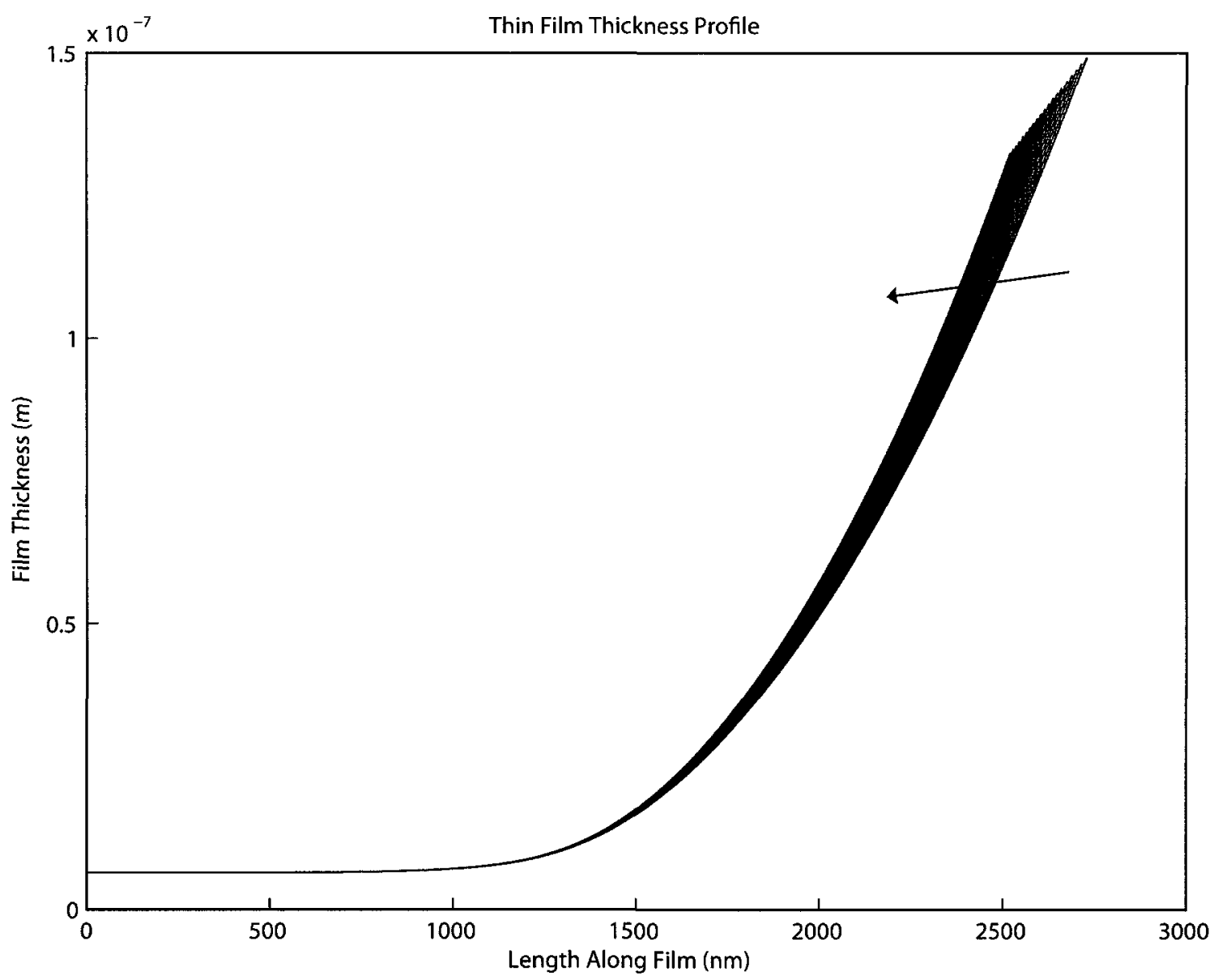

Figure 34: Thin film thickness variance with $\epsilon_{3}$

The thin film interface profiles plotted in Figure 34 are in such proximity that they are nearly indistinguishable. The proximity of the solutions along with the all positive curvature convergence confirms the insensitivity of the third order differential equation to $\epsilon_{3}$. The trend indicated by the directional arrow, shows a decrease in the thin film length with an increasing perturbation size. 


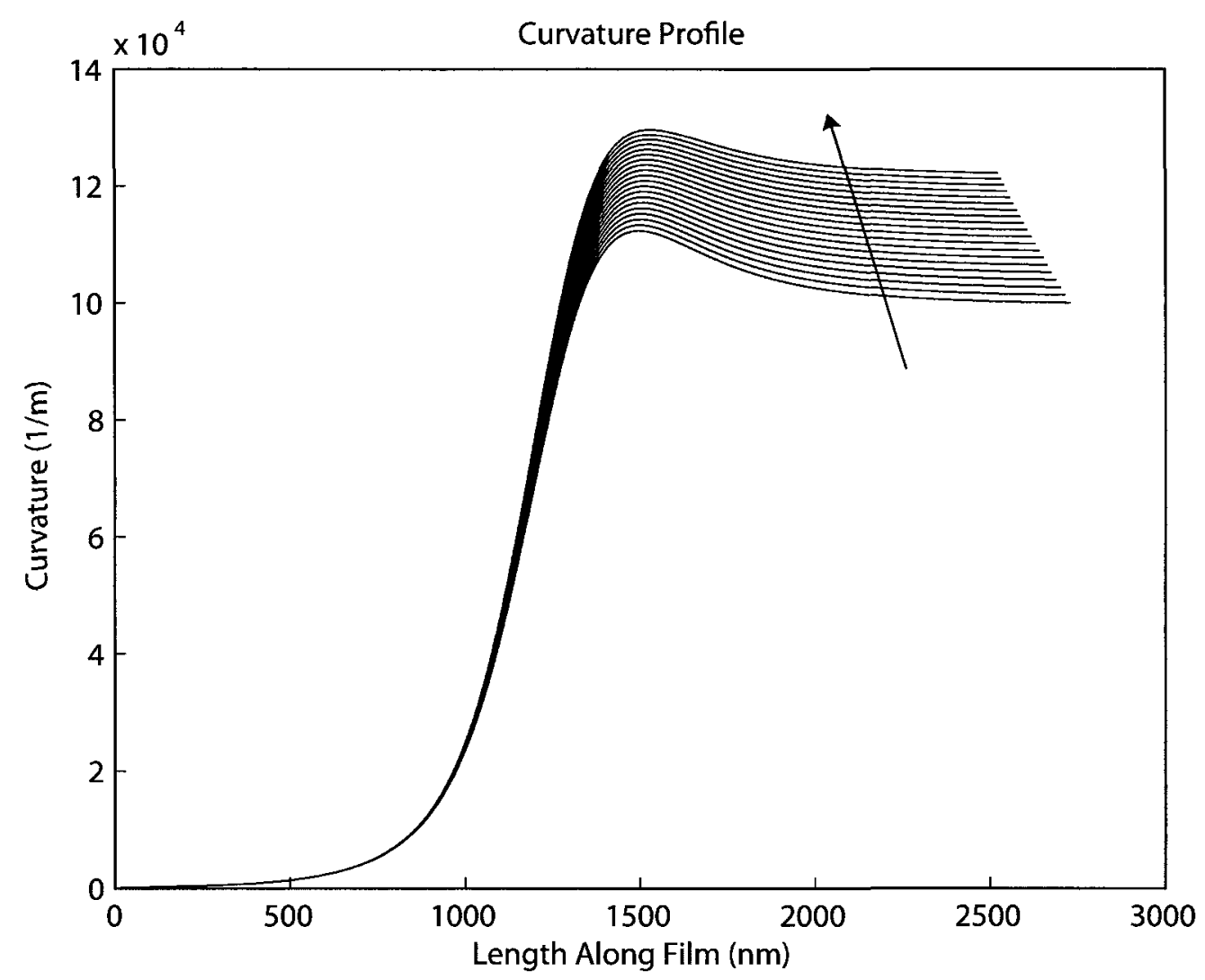

Figure 35: Curvature variance with $\epsilon_{3}$

The curvature results presented in Figure 35, confirm the convergence of all the perturbations to that of positive values. The increase in $\epsilon_{3}$ results in the increase of the converged curvature value, along with a decrease in thin film length.

The coupling of the capillary pressure and curvature profile ensures that the capillary pressure behaves much in the same way as that of the curvature. Although the surface tension is a factor, the interface temperature is almost identical for all perturbations as discussed below. 


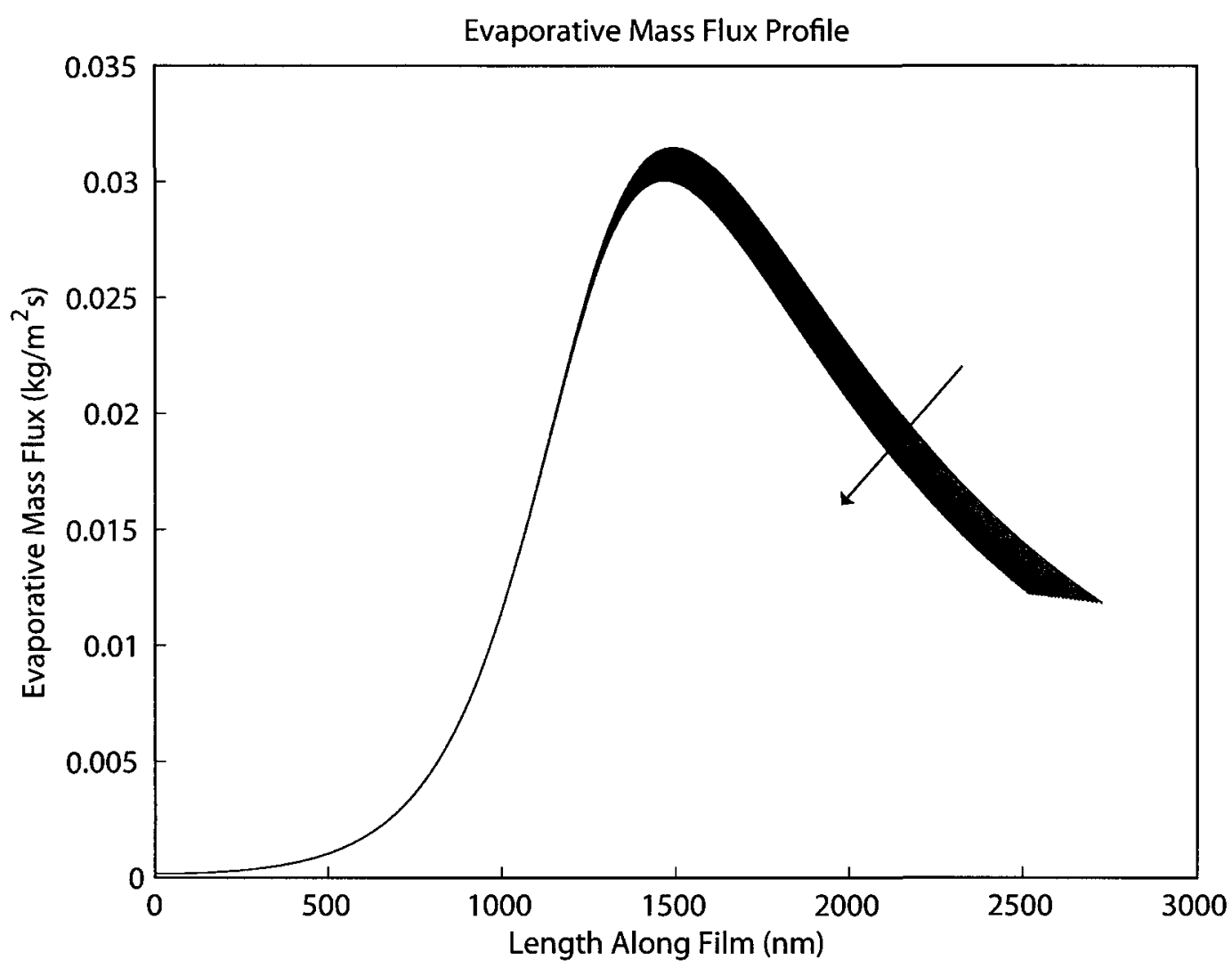

Figure 36: Evaporative mass flux variance with $\epsilon_{3}$

Much like that of the film thickness profile, the evaporative mass flux profiles given in Figure 36, are nearly indistinguishable. The peak of the evaporative mass flux is only slightly affected by the perturbation, and the overall shape of the profile remains consistent. Thus any variance in the perturbation applied to $\delta^{\prime \prime}$ will have very little effect on the evaporative results for the numerical model.

As found before, the liquid pressure gradient is coupled to that of the evaporative mass flux. The results of the liquid pressure gradient are similar in nature to that of the mass flux, with the continued condition of a small lead to that of the liquid pressure gradient peak. 


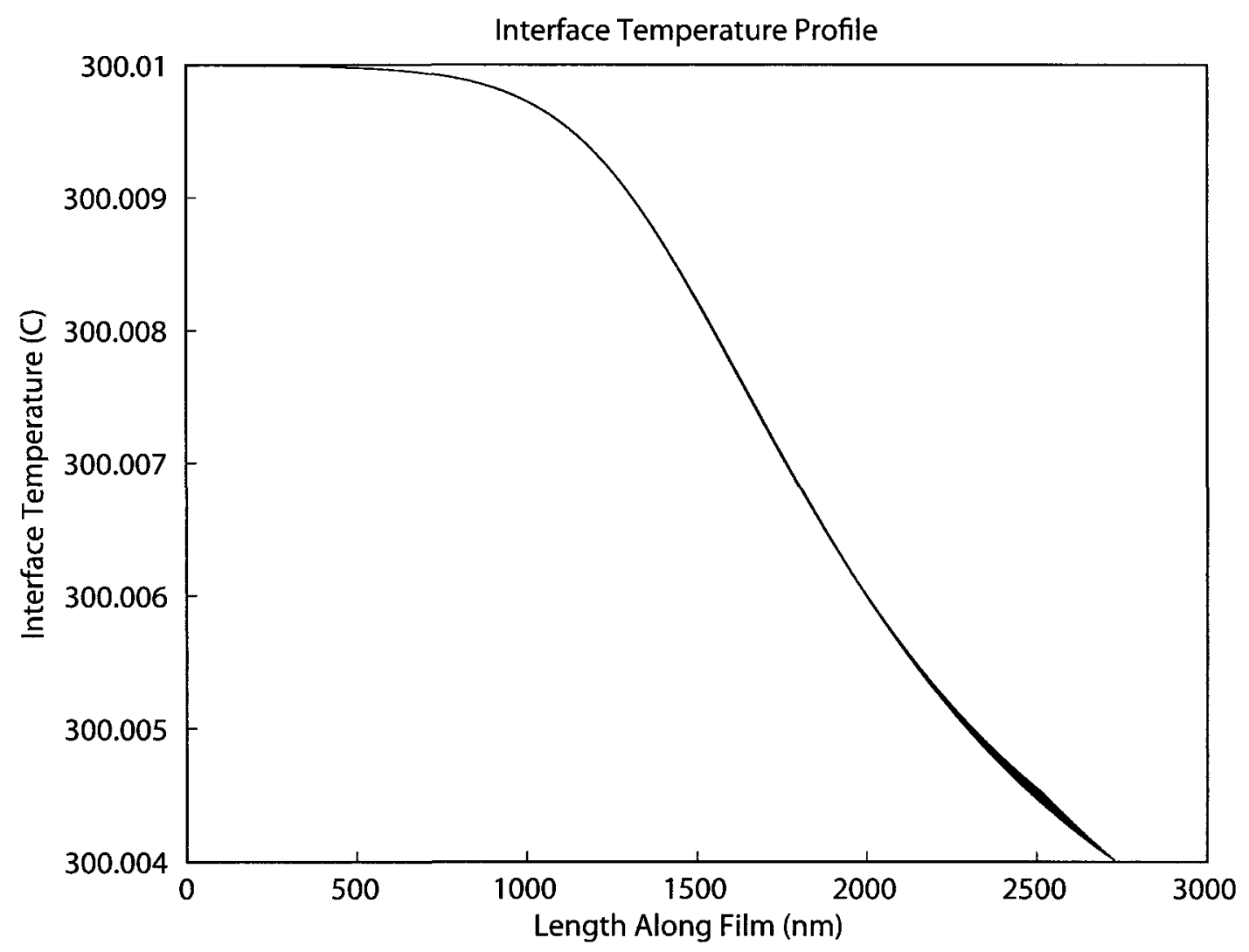

Figure 37: Interface temperature variance with $\epsilon_{3}$

Lastly, the interface temperature profile is shown in Figure 37. The twenty different results are in such proximity that they appear as one line. This proximity indicates that the perturbations applied to $\delta^{\prime \prime}$ has only a small effect on the thin film length.

Thus, the differential equation which describes the evaporating thin film is not sensitive to perturbations applied to $\delta^{\prime \prime}$. This sensitivity observation is only valid for the region of $1 \times 10^{-7} \leq \epsilon_{3} \leq 1 \times 10^{-3}$. This limit is partially based on the assumptions made in the adsorbed region regarding the curvature conditions. 


\subsection{Marangoni effects}

The effects that arise from the presence of an interfacial temperature gradient, known as Marangoni effects, were investigated. The objective of this investigation was to determine if the works of Wang et al. [5] and Park et al. [15], were valid in neglecting the Marangoni effects. The interfacial temperature gradient was numerically modeled both as zero and as a described by Equation (36). The conditions used in the numerical model are given below in Table 6. In solving the Marangoni models, the value of $\delta^{\prime}$ was solved such that a far field constant curvature of $1 / r$ was achieved.

Table 6: Marangoni model input values

\begin{tabular}{|l|c|c|}
\hline \multirow{2}{*}{ Input } & \multicolumn{2}{|c|}{ Value } \\
& $\frac{d T l v}{d x}=0$ & $\frac{d T l v}{d x} \neq 0$ \\
\hline Superheat & $0.01 \mathrm{~K}$ & $0.01 \mathrm{~K}$ \\
Vapour temperature & $300 \mathrm{~K}$ & $300 \mathrm{~K}$ \\
Channel radius & $10 \mu \mathrm{m}$ & $10 \mu \mathrm{m}$ \\
$\epsilon_{1}$ & $0.1 \% \delta_{0}$ & $0.1 \% \delta_{0}$ \\
$\epsilon_{2}$ & $1.2515 \times 10^{-5}$ & $1.2566 \times 10^{-5}$ \\
$\epsilon_{3}$ & $1 \times 10^{-7}$ & $1 \times 10^{-7}$ \\
$\epsilon_{4}$ & 0 & 0 \\
\hline
\end{tabular}

Given the low applied superheat, the differences as a result of interfacial temperature gradients are near zero as shown below in Figures 38 through 41. 


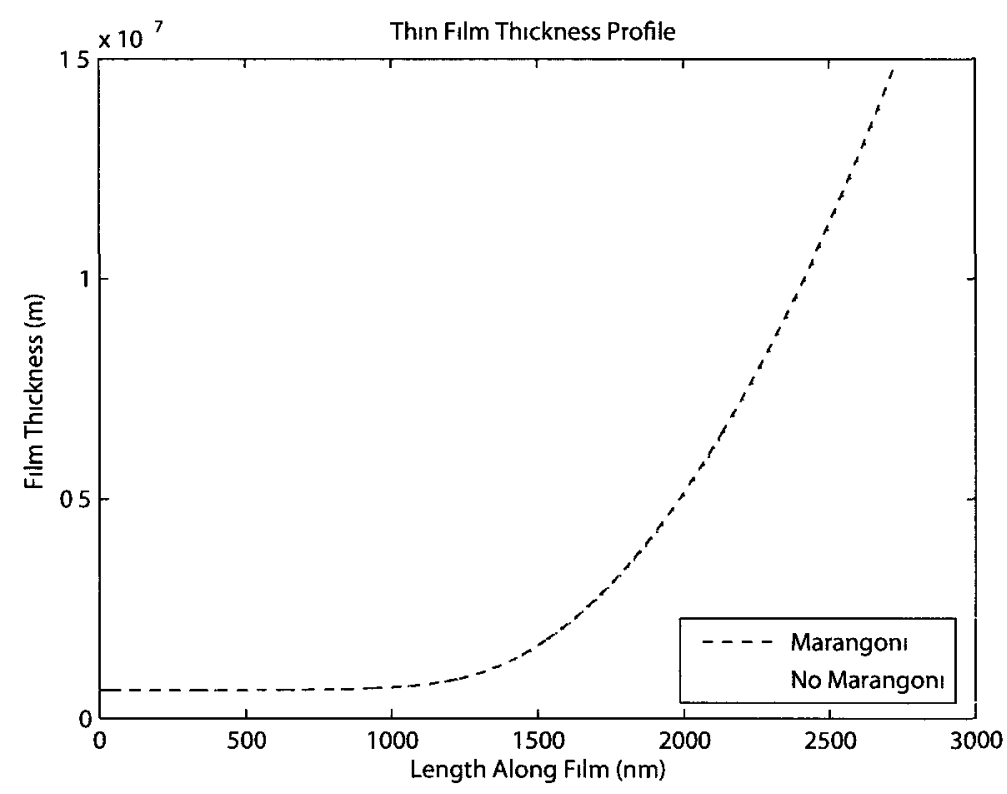

Figure 38: Marangoni effects on thin film thickness

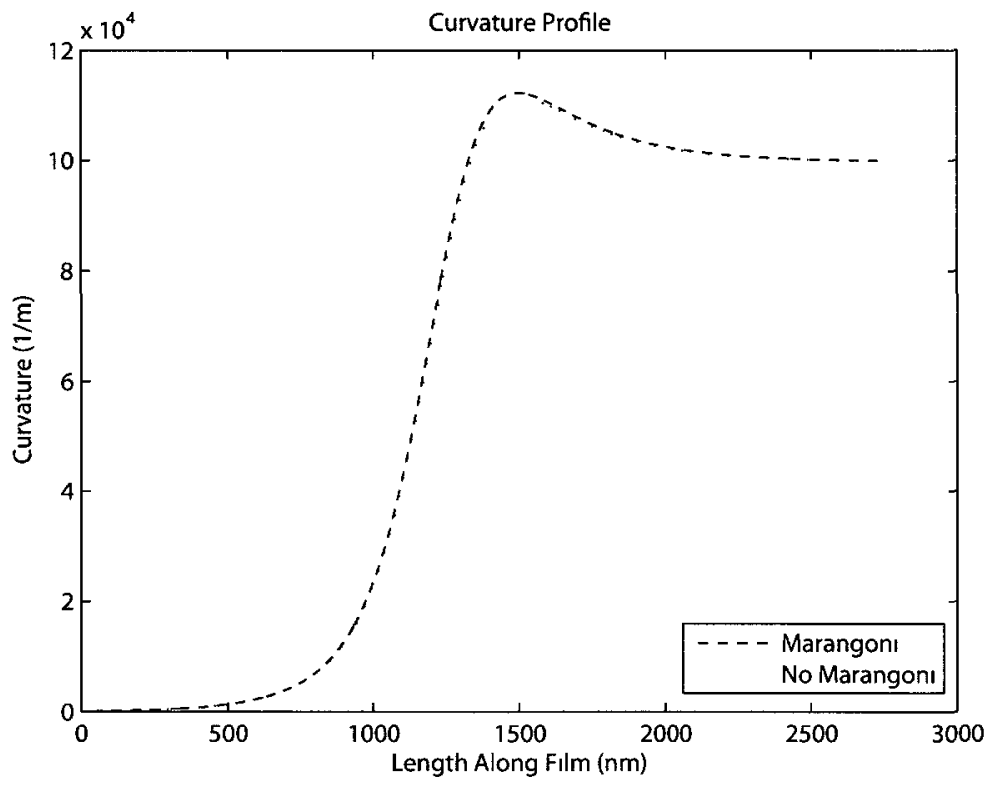

Figure 39: Marangoni effects on far field curvature 


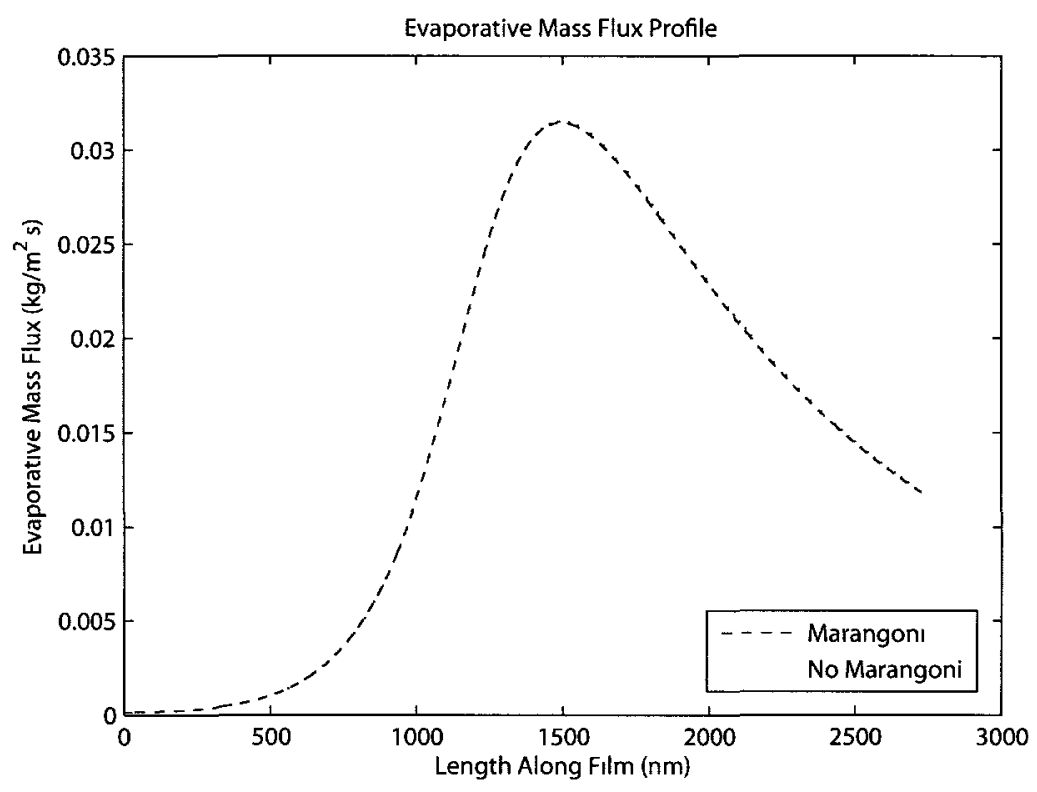

Figure 40: Marangoni effects on evaporative mass flux

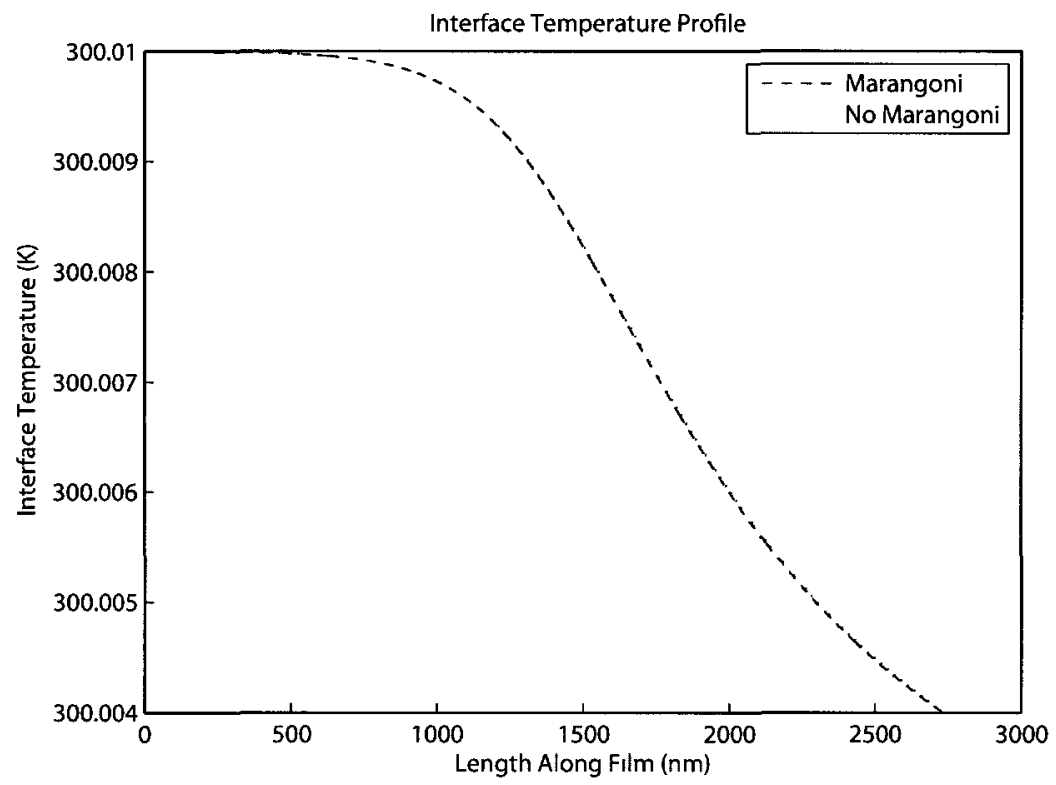

Figure 41: Marangoni effects on interface temperature

The interfacial temperature gradient does not have any effect on the shape or evaporative characteristics of the thin film for this prescribed set of initial conditions. 
Thus a second set of initial conditions were used to determine if there were any differences, resulting from an increase in applied superheat. The results of the new $0.03 \mathrm{~K}$ superheat are given below in Figures 42 through 45 .

Table 7: Marangoni model input values

\begin{tabular}{|l|c|c|}
\hline \multirow{2}{*}{ Input } & \multicolumn{2}{|c|}{ Value } \\
& $\frac{d T l v}{d x}=0$ & $\frac{d T l v}{d x} \neq 0$ \\
\hline Superheat & $0.03 \mathrm{~K}$ & $0.03 \mathrm{~K}$ \\
Vapour temperature & $300 \mathrm{~K}$ & $300 \mathrm{~K}$ \\
Channel radius & $10 \mu \mathrm{m}$ & $10 \mu \mathrm{m}$ \\
$\epsilon_{1}$ & $0.1 \% \delta_{0}$ & $0.1 \% \delta_{0}$ \\
$\epsilon_{2}$ & $1.2548 \times 10^{-5}$ & $1.2601 \times 10^{-5}$ \\
$\epsilon_{3}$ & $1 \times 10^{-7}$ & $1 \times 10^{-7}$ \\
$\epsilon_{4}$ & 0 & 0 \\
\hline
\end{tabular}




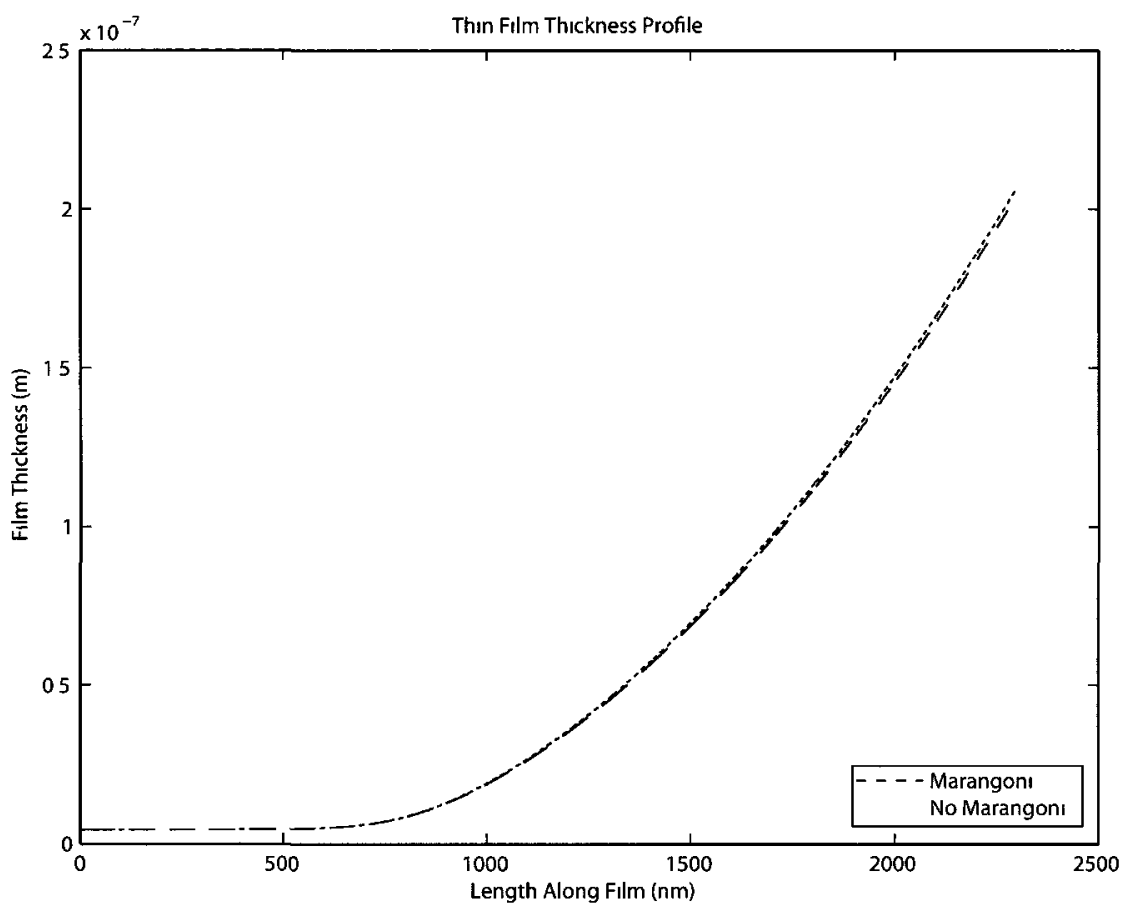

Figure 42: Marangoni effects on thin film thickness

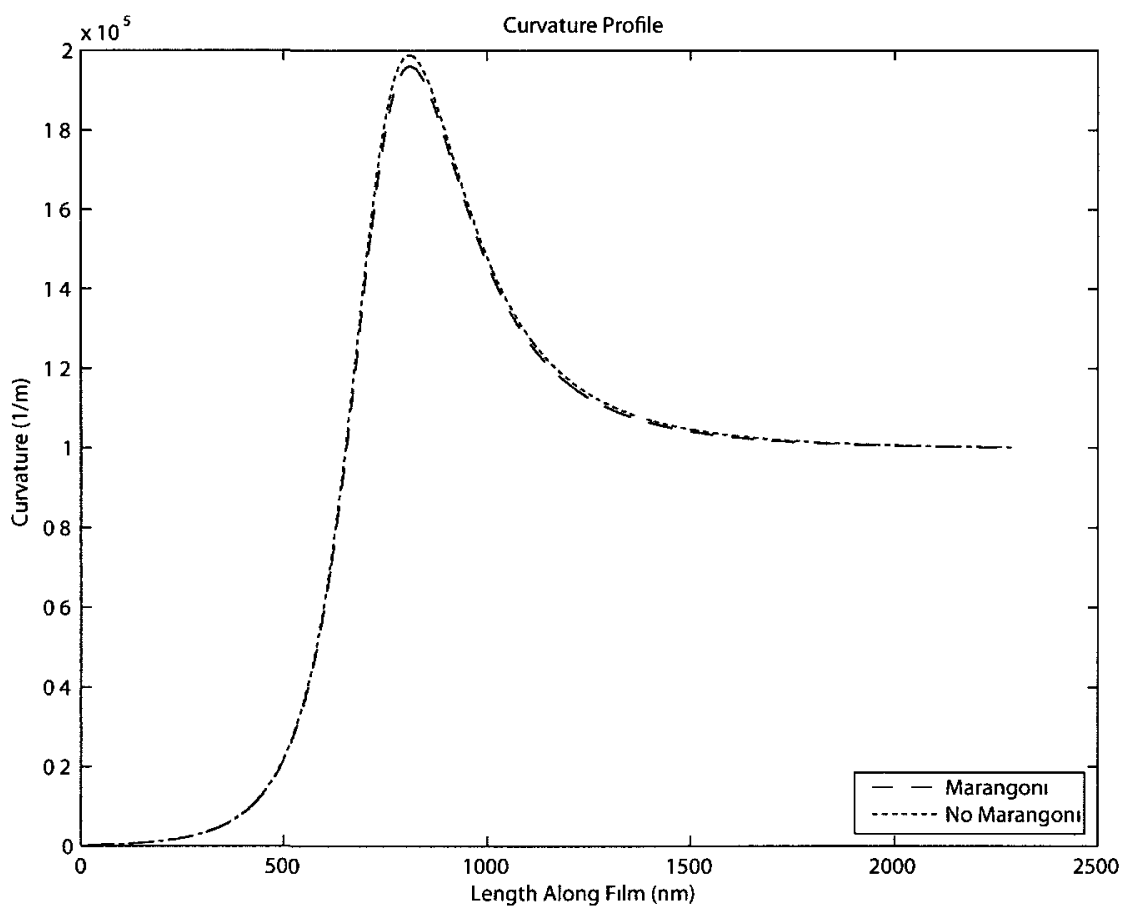

Figure 43: Marangoni effects on far field curvature 


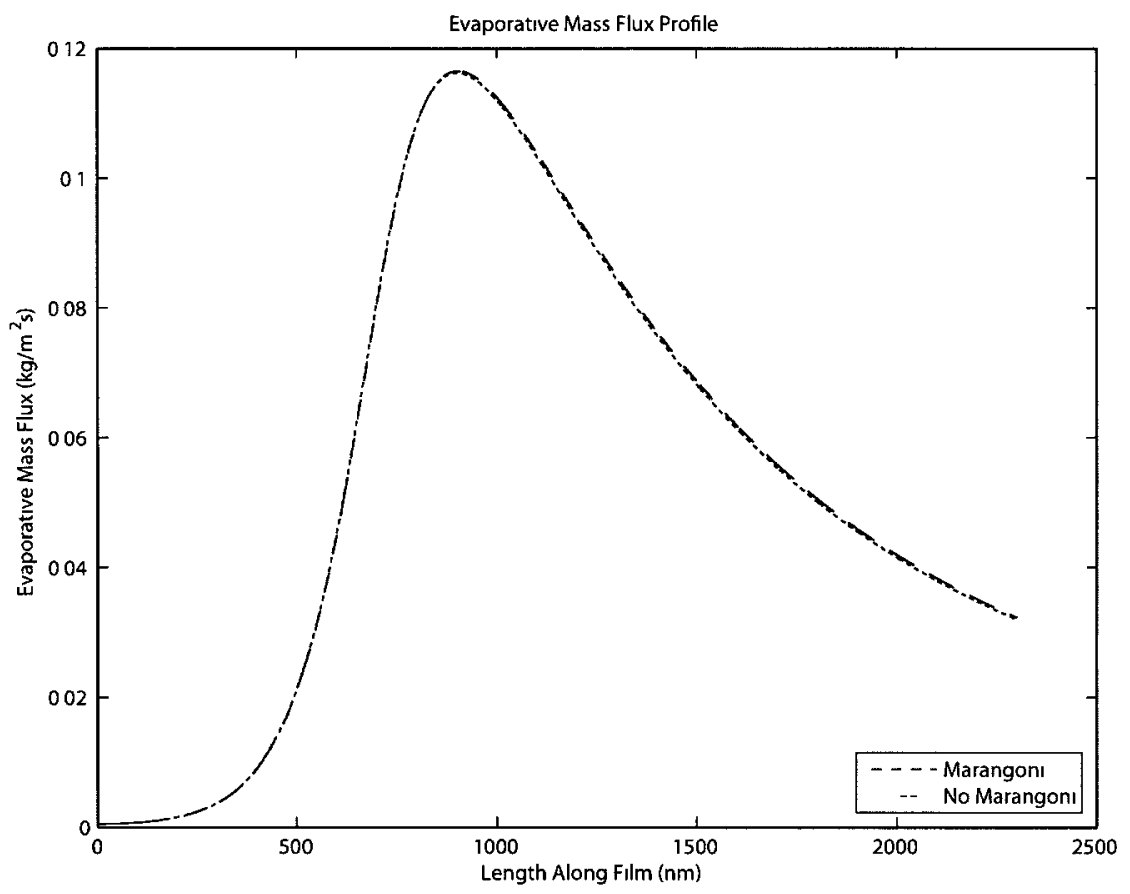

Figure 44: Marangoni effects on evaporative mass flux

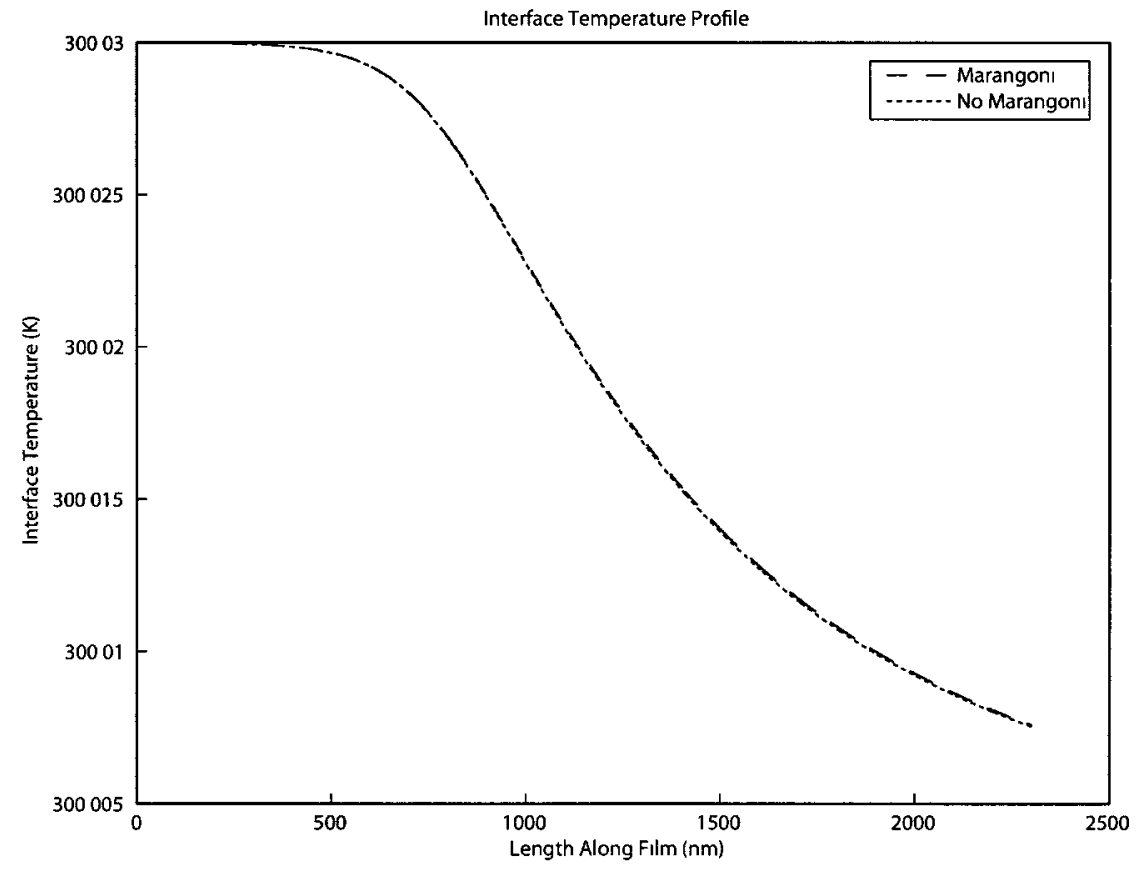

Figure 45: Marangoni effects on interface temperature 
Once again the results are nearly identical for the temperature gradient and nontemperature gradient solutions. The only exception to the identical solutions is that of the curvature overshoot given in Figure 43. The curvature of the thin film begins to show differences in the solutions along the length of the thin film. As the curvature is calculated along the length the overshoot is smaller for that of the solution where the interfacial temperature gradient is not zero.

With the change in applied superheat, the results of the numerical models remained in proximity. Even so, the subtle changes in the curvature overshoot indicate that the interfacial temperature gradient may have a greater effect on the thin film profile with higher applied superheats. With this information, it is possible to speculate that with an increased applied superheat, the surface tension gradients would form Marangoni vortices. If this were the case, the basic assumptions made in the formulation would become invalid, as the Navier-Stokes equation would need to be expanded from a one dimensional flow to that of a two dimensional flow.

Although the differences for the cases provided above are almost negligible, the numerical model used throughout this thesis, includes the presence of an interfacial temperature gradient. This was selected so as to ensure the numerical model accurately represents the physics of the situation being modeled. 


\subsection{Wall slip}

The experimental works of Panchamgam et al. [16], required the presence of a slip condition at the wall so as to match their numerical model to experimental results. This slip condition is related to the application of lubrication theory within the thin film layer itself, for very narrow channels. Thus the potential for wall slip was permitted in the mathematical model and has been set to zero for all previous calculations.

The wall slip that can be accounted for as represented by $\beta$ in Equation (22). The wall slip is thus represented by a length scale, and as reported by Wee et al. [9], is expected to be $4.2 \mathrm{~nm}$ for pentane [9], with the conditions provided in Table 8 . The coordinate system as selected requires that the slip length be entered as a negative value, so as to ensure that the liquid flows toward the adsorbed region. As the effects of the slip length are desired, the numerical model was run for five separate cases where $-4 \times 10^{-9} \leq \beta \leq 0$.

Table 8: Slip length initial conditions

\begin{tabular}{|l|c|}
\hline Input & Value \\
\hline Superheat & $0.01 \mathrm{~K}$ \\
Vapour temperature & $300 \mathrm{~K}$ \\
Channel radius & $10 \mu \mathrm{m}$ \\
$\epsilon_{1}$ & $0.1 \% \delta_{0}$ \\
$\epsilon_{2}$ & Solved for $\kappa=1 / r$ \\
$\epsilon_{3}$ & $1 \times 10^{-7}$ \\
$\epsilon_{4}$ & 0 \\
\hline
\end{tabular}




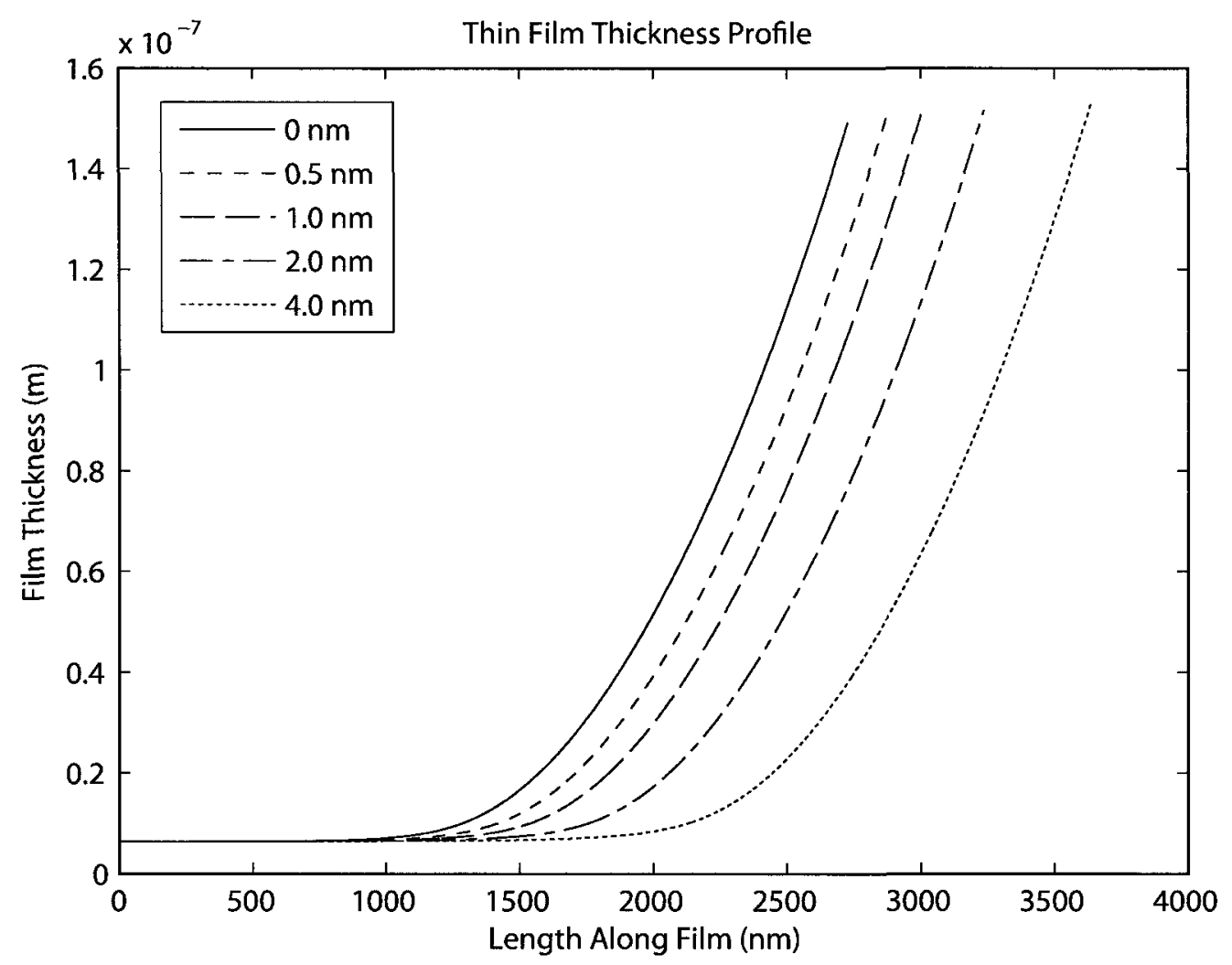

Figure 46: Wall slip effects on thin film thickness

The thin film thickness profiles given in Figure 46, show how the increase in slip length magnitude results in an elongation of the thin film region. This elongation of the thin film is confirmed by the works of Wee et al. [9] and Park et al. [15].

This lengthening process is expected, as the viscous force acting between the wall and liquid has been effectively reduced in magnitude. As such, the liquid retains a greater momentum as it travels toward the adsorbed thin film region. 


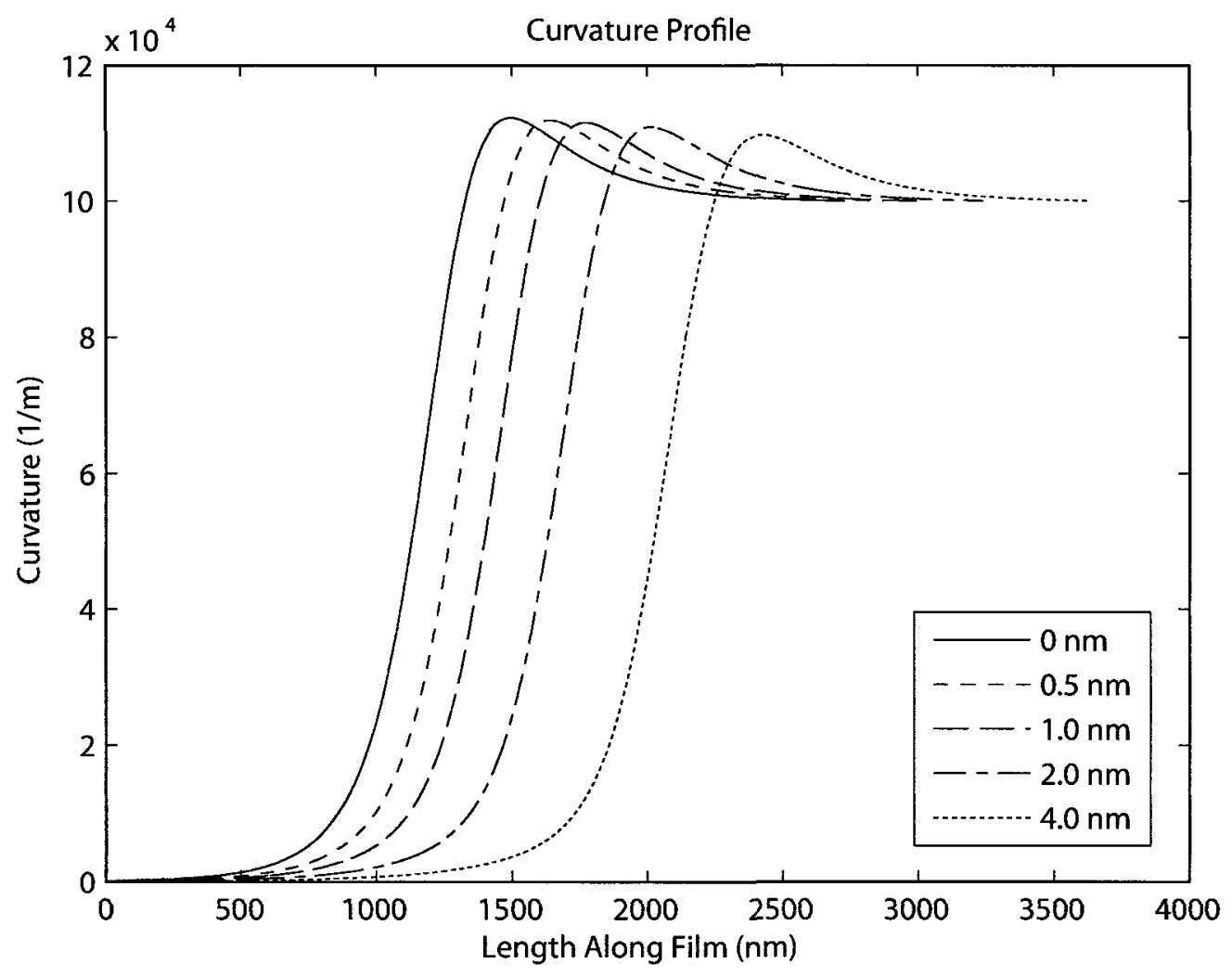

Figure 47: Wall slip effects on the curvature

The curvature profiles in Figure 47, share the same elongation as that of the thin film thickness profiles. The location of the curvature overshoot is observed to shift and slightly decrease in magnitude as the slip length increases. 


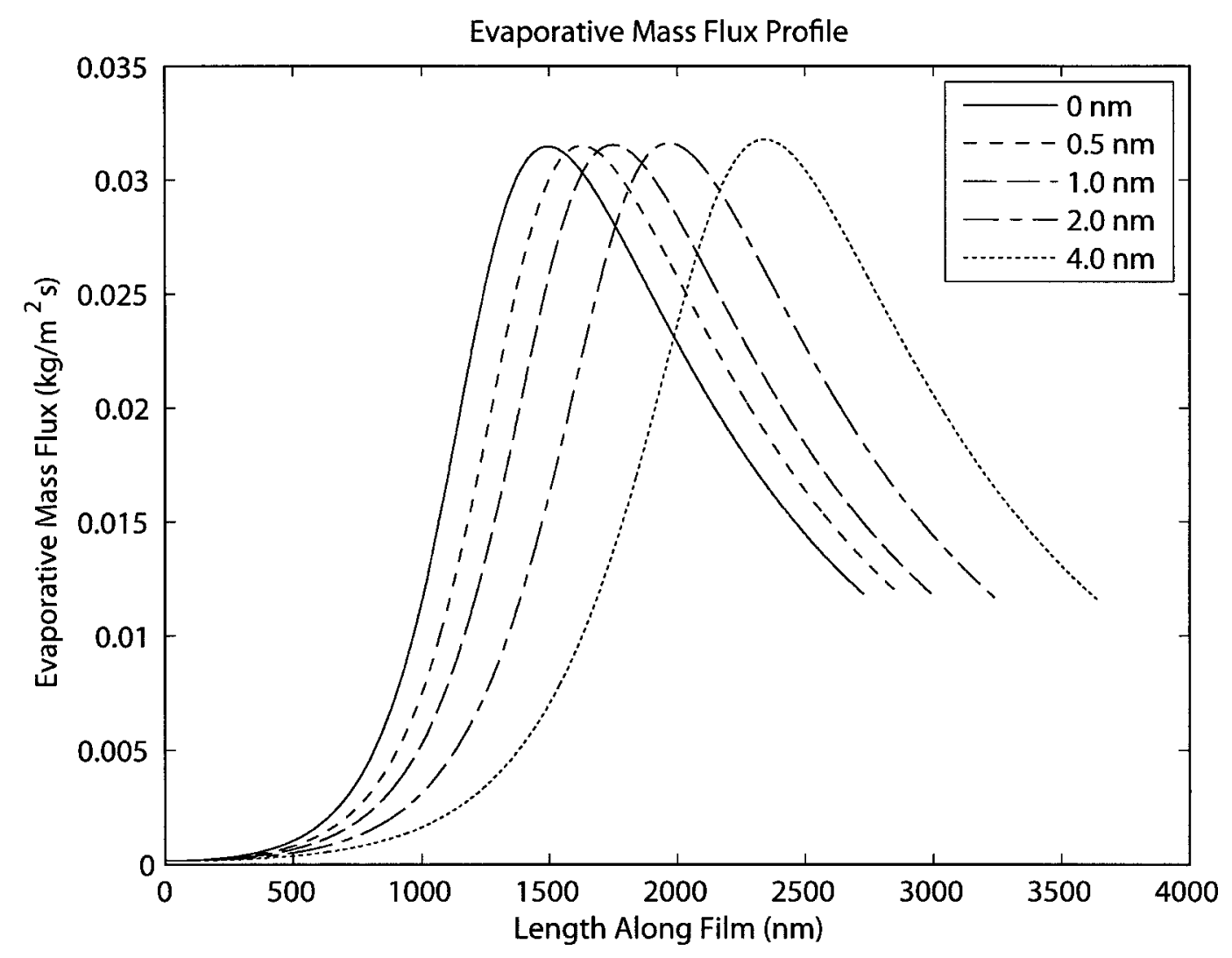

Figure 48: Wall slip effects on the evaporative mass flux

The mass flux profiles illustrated in Figure 48, have maintained their magnitude with some increase in breadth. The origin of the mass flux is almost zero lending to the requirement of no evaporation in the adsorbed region. With the extension of the thin film length, the growth of the thin film thickness is delayed thus allowing for the disjoining pressure to dominate further into the thin film region of the evaporating meniscus. 


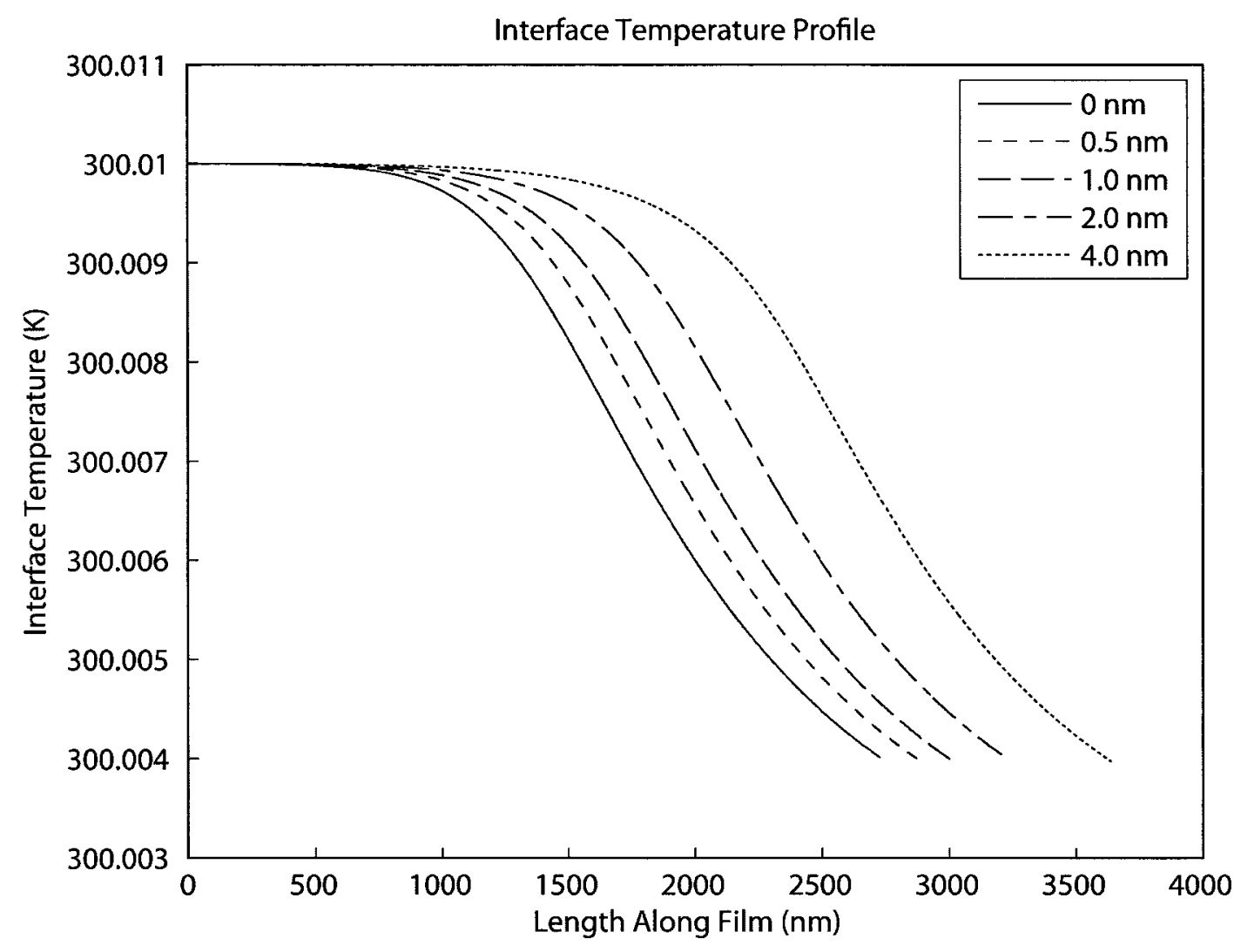

Figure 49: Wall slip effects on the interfacial temperature profile

The interface temperature profiles as shown in Figure 49, exhibit the same characteristics as those mentioned earlier. The effects of the wall slip have permitted the formation of a longer transition region which the disjoining pressure dominates. This delay in thin film growth subsequently leads to the near uniformity in temperature until such point that the evaporative cooling can reduce the interface temperature.

The wall slip length allows for the formation of an elongated thin film region for evaporation to occur. The increase in evaporative mass flux discussed by Panchamgam et al. [16], may come from the increased evaporative area as well as the increase in breadth of the evaporative mass flux profile as shown in Figure 48. 


\subsection{Different fluids}

The mathematical model developed makes use of the non-polar form of the disjoining pressure equation. As such, only non-polar fluids are to be simulated such as pentane which has been used throughout all of the previous cases.

To ensure that the model is consistent, a second non-polar fluid, such as octane can be used. This change in fluid is meant to represent the potential for applications that may have different environmental or operational conditions.

The results presented herein use the conditions as outlined in Table 9. To maintain consistency the conditions used for octane are the same as those used previously for the pentane. The value stated for $\delta^{\prime}$ is solved such that the far field curvature is the desired constant $1 / r$.

Table 9: Pentane and octane initial conditions

\begin{tabular}{|l|c|c|}
\hline \multirow{2}{*}{ Input } & \multicolumn{2}{|c|}{ Value } \\
& Pentane & Octane \\
\hline Superheat & $0.01 \mathrm{~K}$ & $0.01 \mathrm{~K}$ \\
Vapour temperature & $300 \mathrm{~K}$ & $300 \mathrm{~K}$ \\
Channel radius & $10 \mu \mathrm{m}$ & $10 \mu \mathrm{m}$ \\
$\epsilon_{1}$ & $0.1 \% \delta_{0}$ & $0.1 \% \delta_{0}$ \\
$\epsilon_{2}$ & $1.2566 \times 10^{-5}$ & $9.2493 \times 10^{-6}$ \\
$\epsilon_{3}$ & $1 \times 10^{-7}$ & $1 \times 10^{-7}$ \\
$\epsilon_{4}$ & 0 & 0 \\
\hline
\end{tabular}




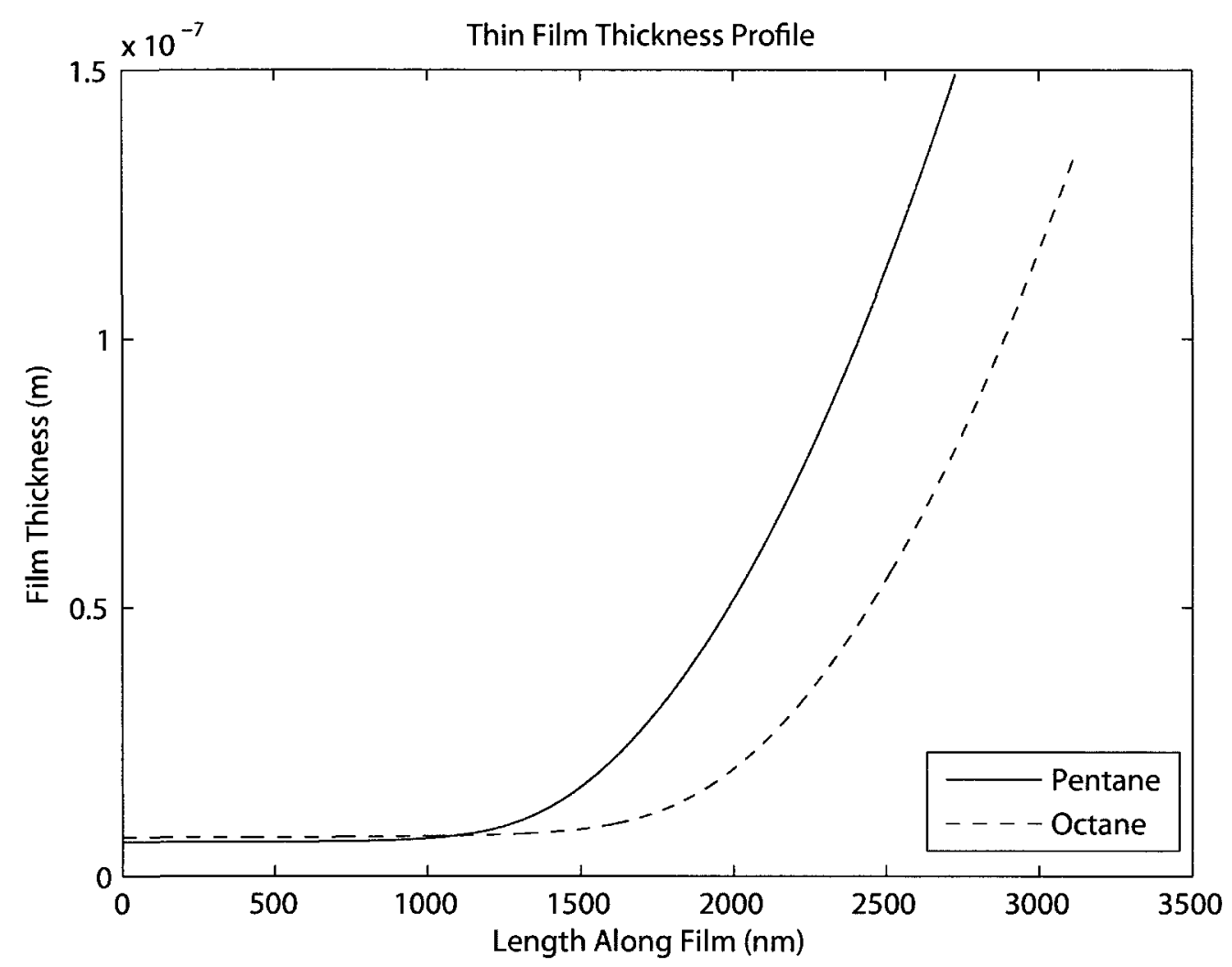

Figure 50: Pentane and octane thin film thickness

The progressive growth of the thin films for each fluid, as shown in Figure 50, are quite different from each other. The differences begin at the start of the thin film region.

The thickness at the start of the thin film is different as a result of the differences in the Hamaker constant and the properties of fluid itself. As with each fluid-solid set, the Hamaker constant is different. This difference in the Hamaker constant, fluid properties and thin film thickness allow for the disjoining pressure to have a greater dominance for the octane, than that of the pentane. This in part accounts for the increased length of the thin film region. 


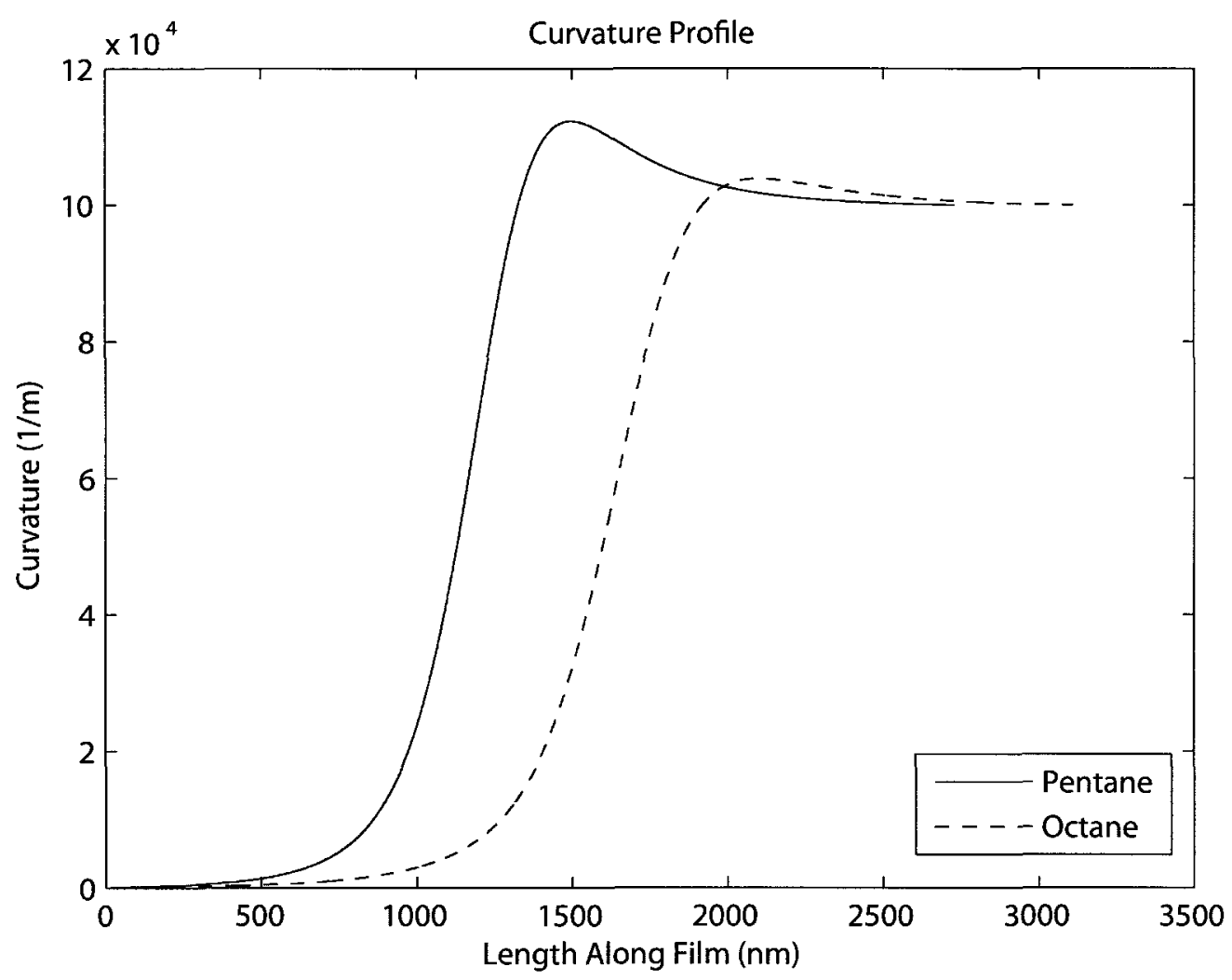

Figure 51: Pentane and octane curvature profiles

The curvature profiles shown above in Figure 51, have a difference in the location and magnitude of the curvature overshoot. In addition, the slope of the curvature profile is slightly less for octane than pentane. 


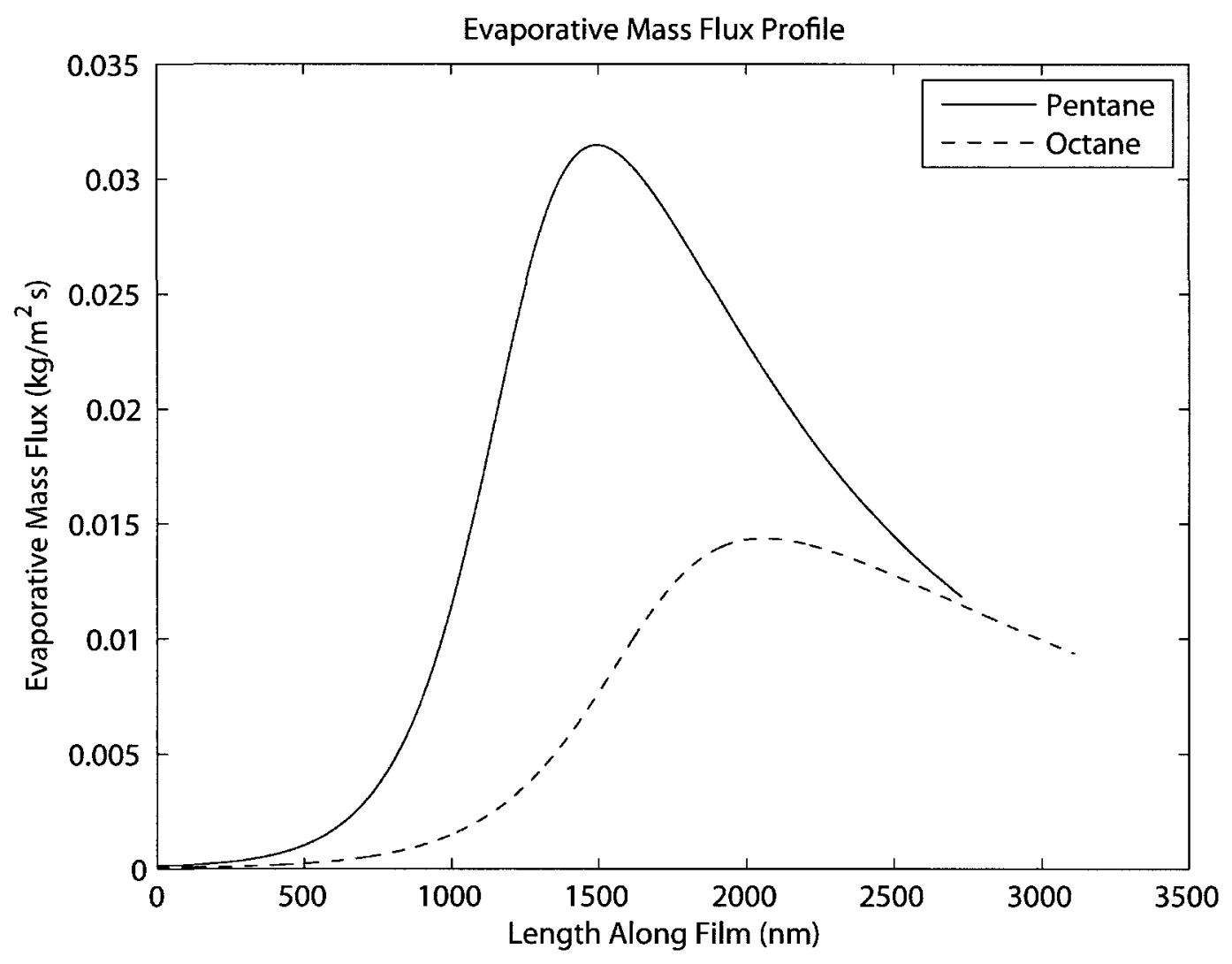

Figure 52: Pentane and octane evaporative mass flux profiles

The evaporative mass flux experienced by each fluid as given in Figure 52, is quite different for each fluid. The pentane once again has a high a pronounced peak and the octane has a diminished peak. The shape and location of the peaks for each fluid coincide with the shape and location of the curvature overshoot. 


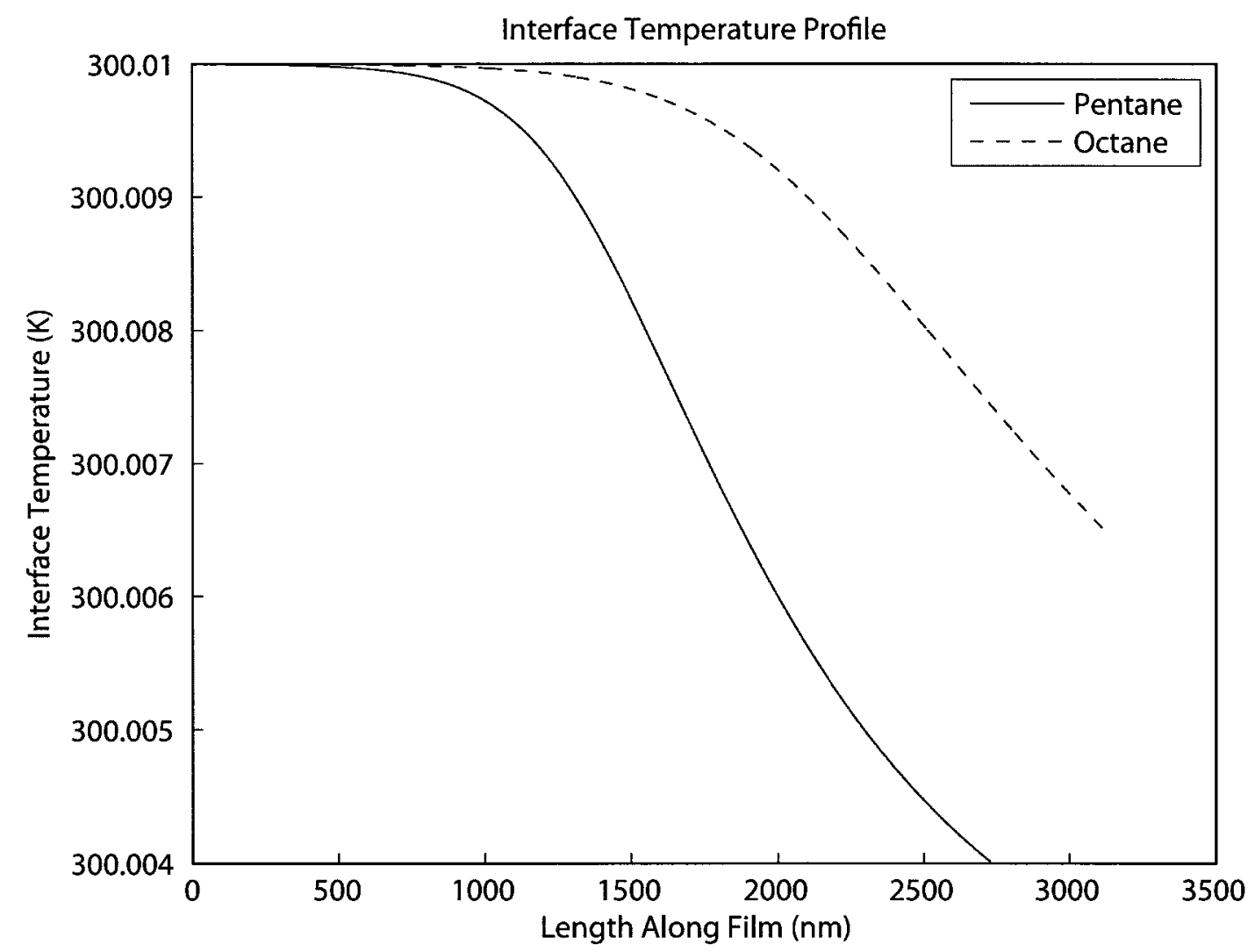

Figure 53: Pentane and octane interface temperature profiles

The interface temperature profiles provided in Figure 53, are as expected. The octane temperature retains a higher value as a result of the extension of the thin film length. With regards to the shape, the pentane temperature profile appears to drop forming a convex, then later transitioning to a concave. This shift in temperature is not observed in the octane solution. 


\subsection{Different channel widths}

In engineering applications it is important to understand how geometry can attribute to the changes in evaporation properties. As with grooved evaporators in heat pipes, the groove dimension can influence the heat transport capacity, provided a geometric relation is present.

To determine how channel width affects the evaporative characteristics of the thin film region a set of channels were selected. For use in the numerical model the channel radius is used instead of the channel width. This is done so as to calculate the desired far field curvature. In total, five different channel radii were investigated, ranging from $2.5 \mu m \leq r \leq 40 \mu m$.

The initial conditions used for the simulation are given below in Table 10. The fluid used is once again pentane.

Table 10: Channel width initial conditions

\begin{tabular}{|l|c|}
\hline Input & Value \\
\hline Superheat & $0.01 \mathrm{~K}$ \\
Vapour temperature & $300 \mathrm{~K}$ \\
Channel radius & $2.5 \mu m \leq r \leq 40 \mu m$ \\
$\epsilon_{1}$ & $0.1 \% \delta_{0}$ \\
$\epsilon_{2}$ & Solved for $\kappa=1 / r$ \\
$\epsilon_{3}$ & $1 \times 10^{-7}$ \\
$\epsilon_{4}$ & 0 \\
\hline
\end{tabular}




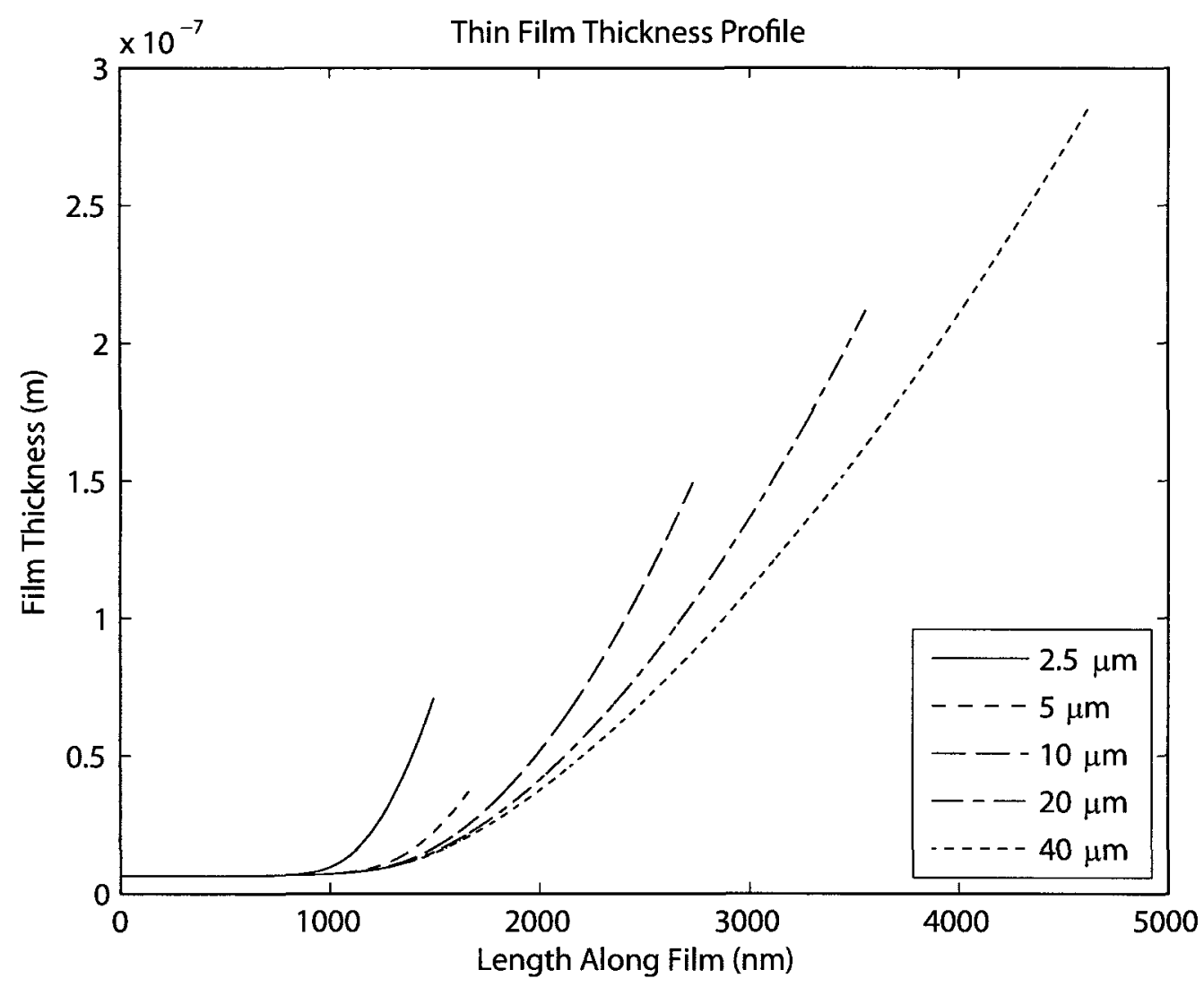

Figure 54: Channel radius effects on thin film thickness

The thin film thickness as given above in Figure 54, illustrates how the film thickness varies with channel width. For all of channel radii greater than $2.5 \mu m$, they begin to increase in thickness near the same point.

The second exception in Figure 54, is the termination point of the $5 \mu \mathrm{m}$ radius channel. The other four channel radii terminate so as to form the four points of a straight line. This may indicate a problem with the termination conditions for the $5 \mu m$ channel.

Despite the differences in the termination point of the thin film thickness, the trend shows that an increase in channel width leads to the lengthening of the thin film. This trend has been observed in the numerical models of Wang et al. [5], Zhao et al. [3] and the experimental results of $\mathrm{Qu}$ et al. [11]. 


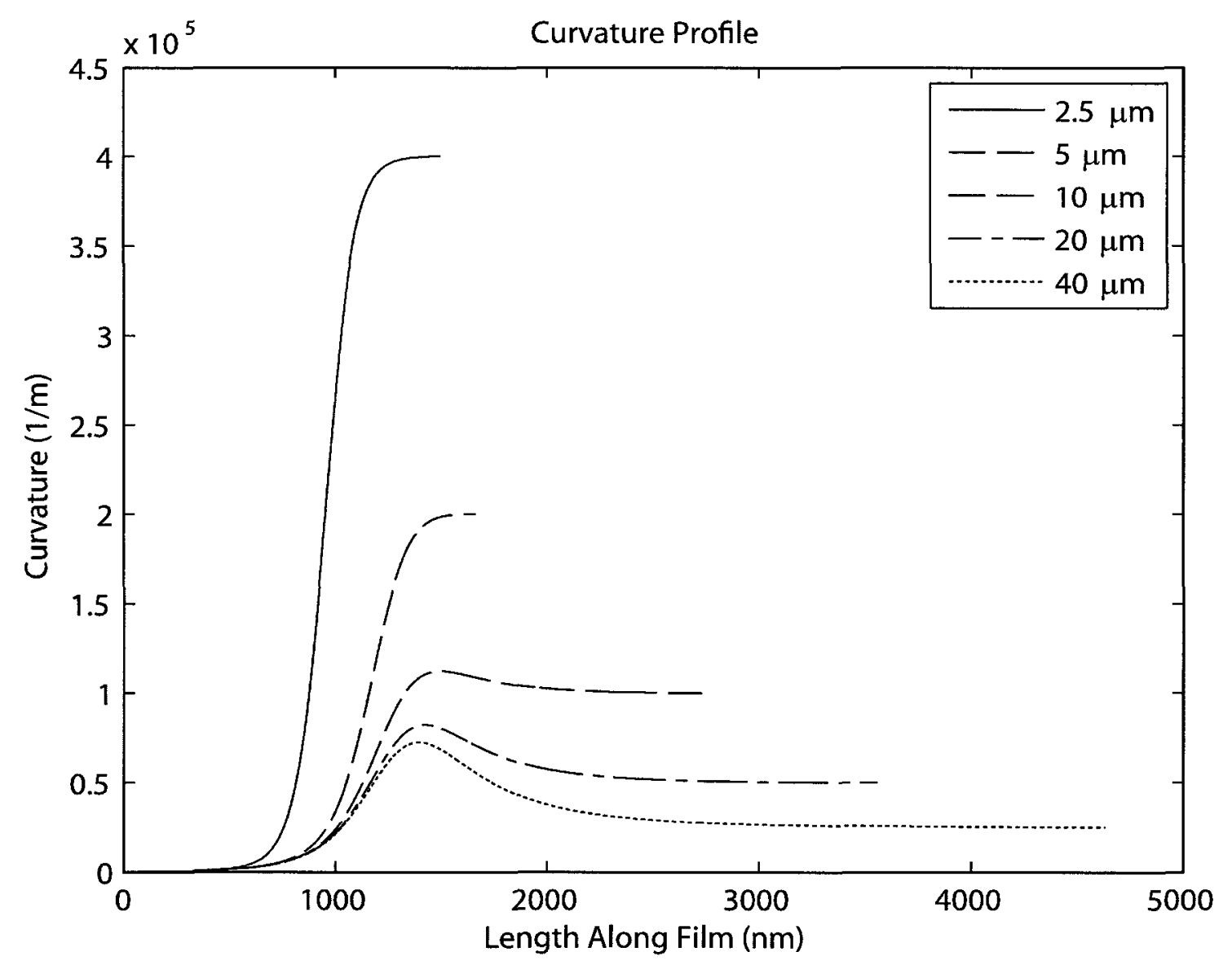

Figure 55: Channel radius effects on local curvature

The curvature profiles for each channel width as provided in Figure 55, show how the numerical model converges. As desired the far field curvature for each of the channels corresponds to a $1 / r$ solution.

The shape and gradient of the curvature shares the turning point with that of the film thickness profiles discussed previously. It appears that the $2.5 \mu m$ channel, undergoes a curvature change separate from the other channel widths. The curvature climbs rapidly, and then converges without any curvature overshoot. 
For the remaining channel widths, they all start and follow a similar path. Around $1000 \mathrm{~nm}$, the curvature profiles for each channel begins to separate, as they progress to their various final curvatures. For the $5 \mu \mathrm{m}$ channel, no curvature overshoot is observed and reaches a constant value quickly.

As the channel width increases past $5 \mu m$, an overshoot in the curvature is observed. The reason for the overshoot is currently unknown and is considered to be a characteristic of the differential equation. However this peak in curvature was experimentally measured in the works of Panchamgam et al. [16].

In the trends associated with the channel width, it is possible to see that the far field constant curvature will tend toward a limit of zero. This limit of zero comes from the $1 / r$ curvature condition, where the radius tends towards infinity lending to the Young-Laplace solution for a large reservoir. 


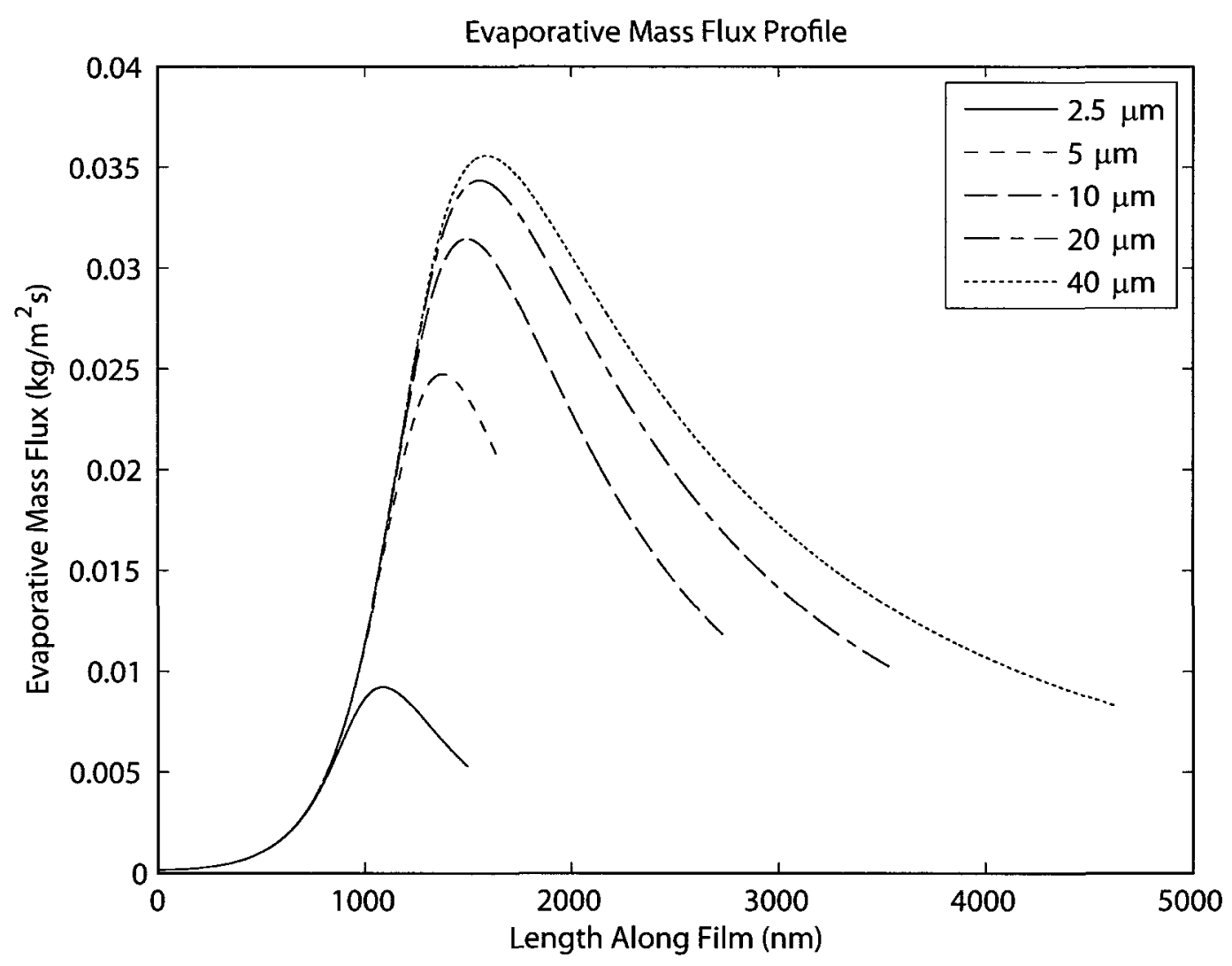

Figure 56: Channel radius effects on evaporative mass flux

The evaporative mass flux profiles given in Figure 56, show a significant shift in shape and magnitude. The solutions are consistent in how the mass flux starts at zero in the region nearest the adsorbed layer.

As the mass flux increases toward the peak, the various channel widths branch off toward their respective maximums. The trend observed is similar to that of the curvature and the potential limit. In this case, it appears that the evaporative mass flux indicates a potential plateau in the available evaporation. The tendency for the evaporative mass flux to increase is confirmed by the numerical model conducted by Wee et al. [9]. 
The evaporative capability of the different channel widths can be represented by the area under the curve. Thus, the greatest evaporative heat transfer is for that of the widest channel modeled.

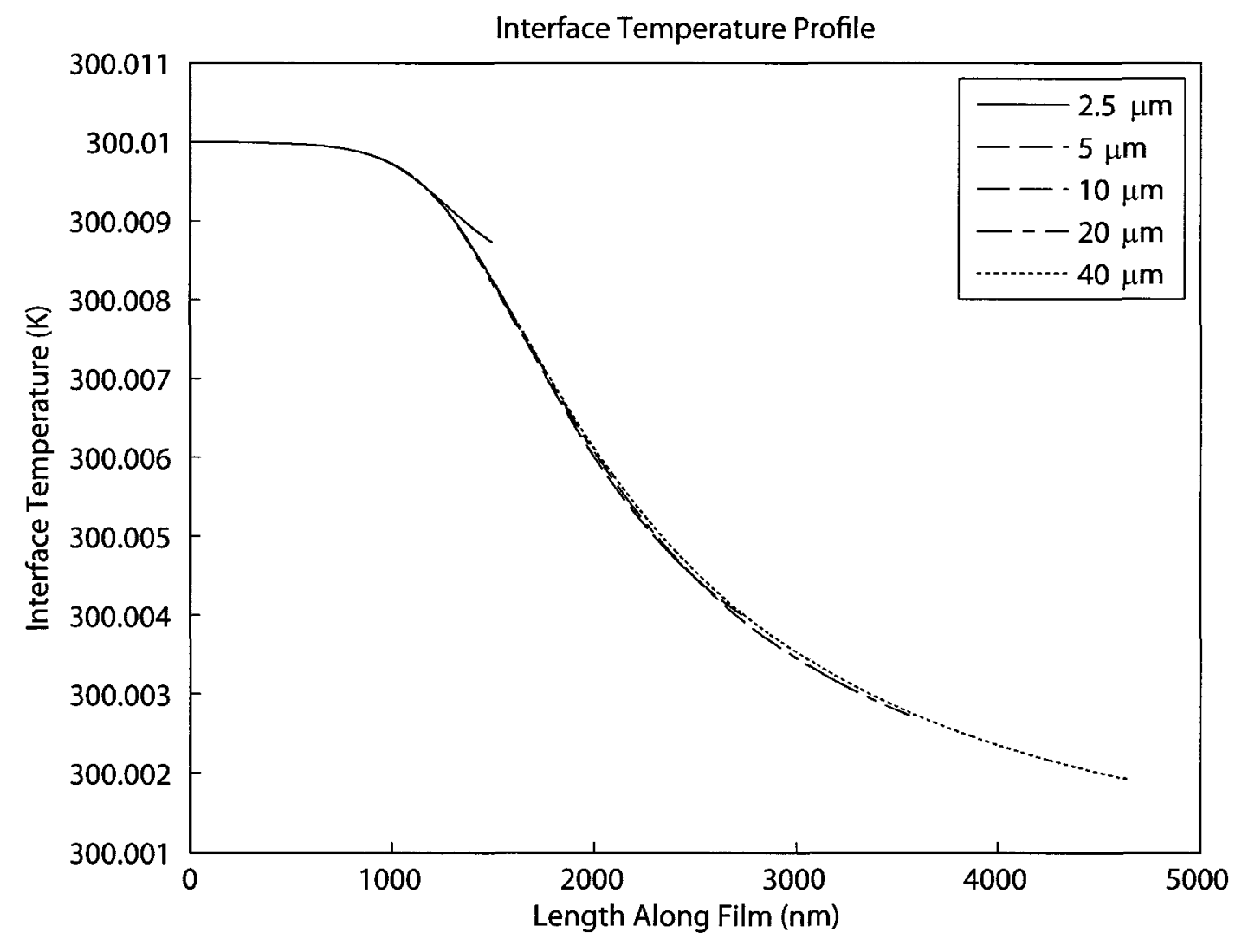

Figure 57: Channel radius effects on interface temperature

Last is the interface temperature profile as given in Figure 57. The temperature profiles for the interface are nearly identical for all of the channel widths. 


\subsection{Different superheats}

The final set of data collected was for that of a range of applied superheats. The superheat was incrementally increased from $0.01 \mathrm{~K}$ to $0.05 \mathrm{~K}$ with a total of five values tested. The highest applied superheat modeled was that of $0.05 \mathrm{~K}$ because of numerical instability resulting from the initial perturbation size selection. This instability is reasonable, as the slope of the thin film at the start is expected to increase as the thin film region becomes smaller. The conditions used for the model, are given below in Table 11, with the value of $\delta^{\prime}$ solved such that the far field curvature achieved a $1 / r$ value.

Table 11: Initial conditions for varied superheats

\begin{tabular}{|l|c|}
\hline Input & Value \\
\hline Superheat & $0.01 \rightarrow 0.05 \mathrm{~K}$ \\
Vapour temperature & $300 \mathrm{~K}$ \\
Channel radius & $10 \mu m$ \\
$\epsilon_{1}$ & $0.1 \% \delta_{0}$ \\
$\epsilon_{2}$ & Solved for $\kappa=1 / r$ \\
$\epsilon_{3}$ & $1 \times 10^{-7}$ \\
$\epsilon_{4}$ & 0 \\
\hline
\end{tabular}

Of the initial conditions used, the percentage system for the $\epsilon_{1}$ perturbation is important. This method of perturbation was found after a variety of trial and errors. This percentage based perturbation ensures that the perturbation is based off the adsorbed thickness. 
For the variance of applied superheat, the adsorbed thickness changes accordingly. This change in adsorbed film thickness is where the percentage type of perturbation comes to be of benefit. It ensures a consistent perturbation size despite the changes in adsorbed thickness. Thus minimizing the undesired effects, stemming from the sensitivities of the governing differential equation as discussed earlier in the parametric study.

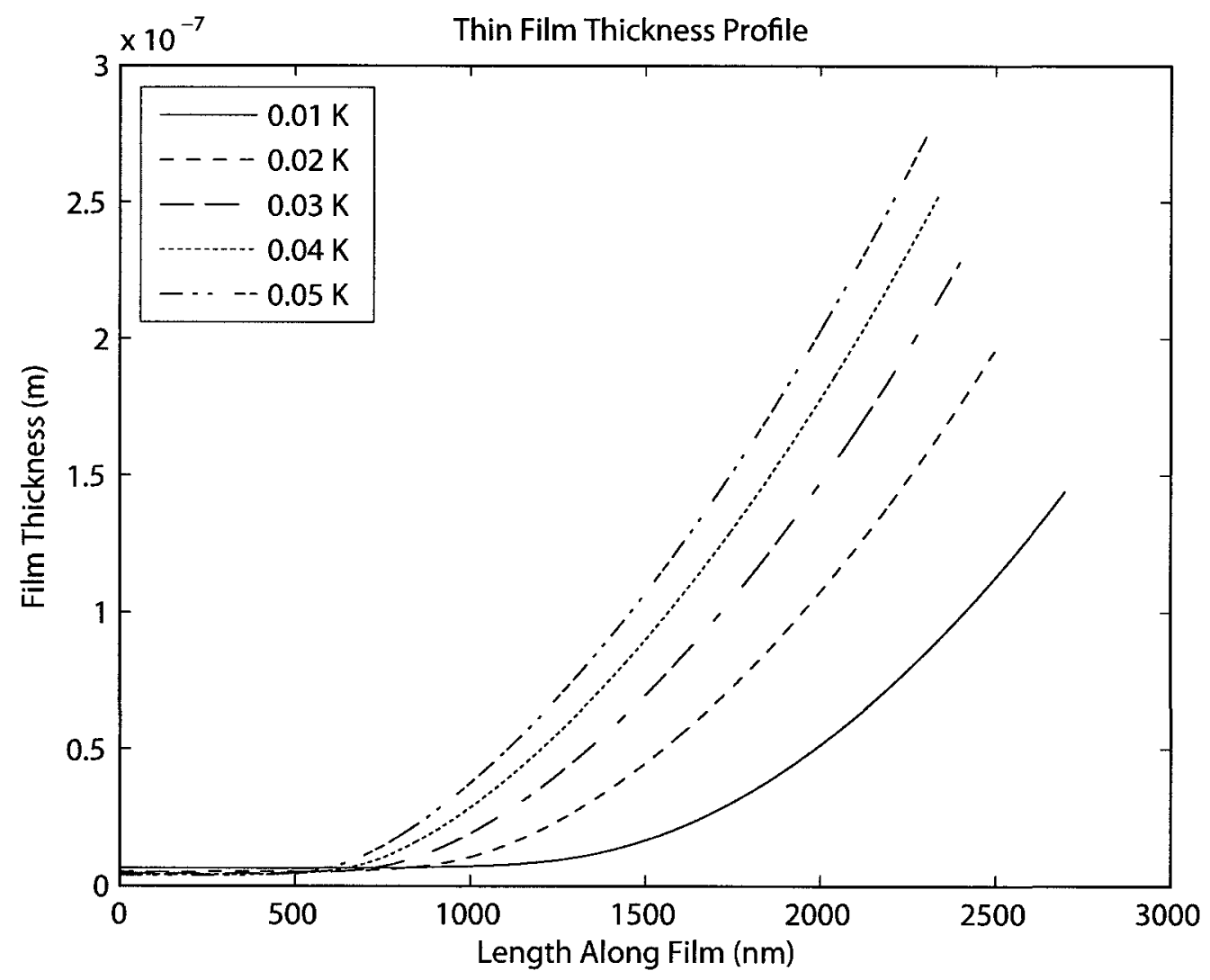

Figure 58: Superheat effects on thin film thickness

The thin film thickness profiles for the applied superheats are shown in Figure 58. The start of the thin film region exhibits a shift in the adsorbed layer thickness as expected. 
The film thickness increases with the increase of applied superheat, thus effectively forming ever shallower menisci. This phenomenon is documented in other numerical models and experimental data collected $[3,5,9,11,35]$.

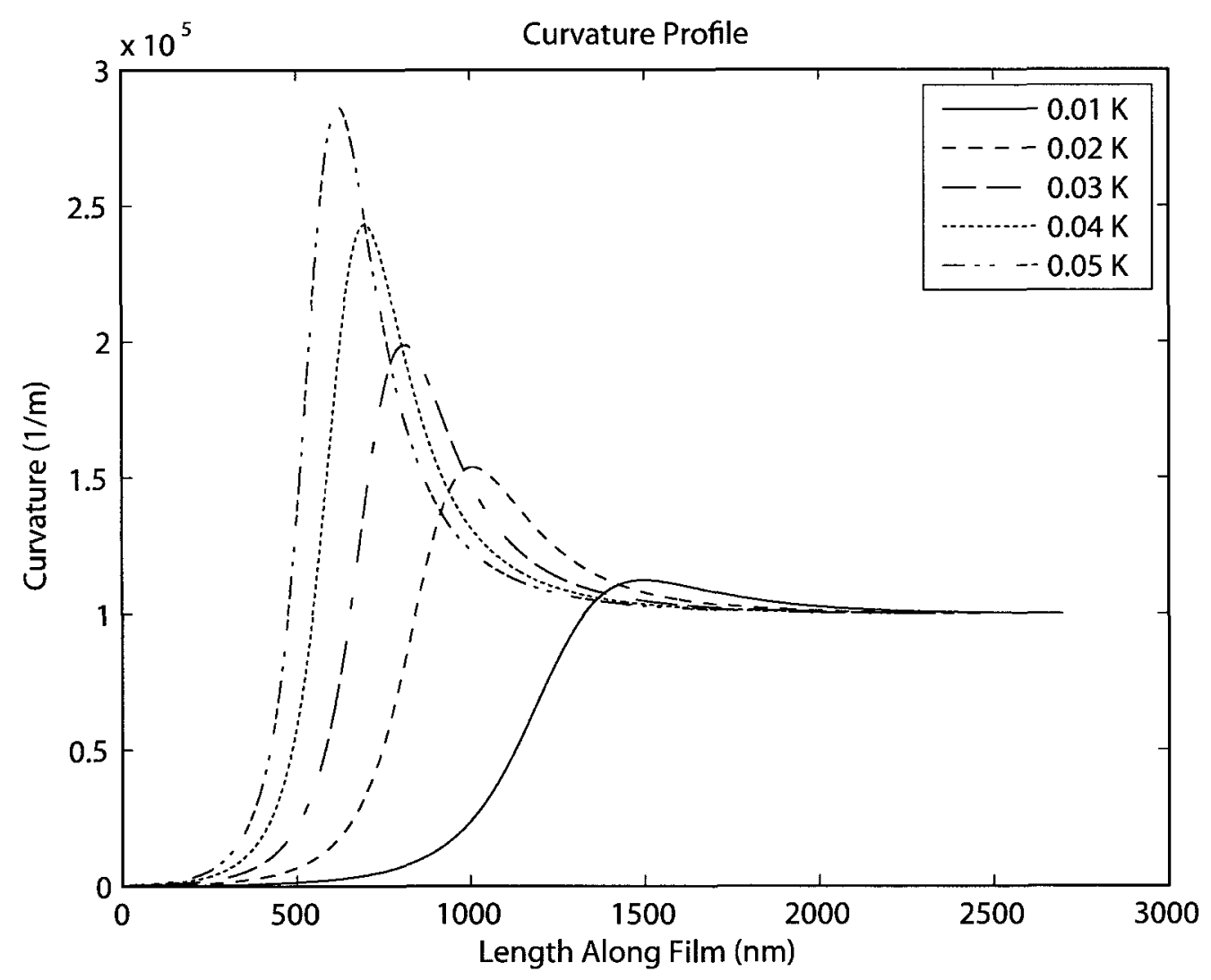

Figure 59: Superheat effects on curvature

The curvature profiles for the different applied superheats shown in Figure 59, illustrate a large sensitivity to variance in applied superheats. With the increase in applied superheat, the curvature tends to both shorten the thin film length and increase the curvature overshoot.

The nature of the curvature overshoot as a function of superheat variance is documented numerically and experimentally $[3,16,35]$. Of the experimental data collected, the curvature peak change in superheat is observed in the transient aspects of the switching on of a heater. 


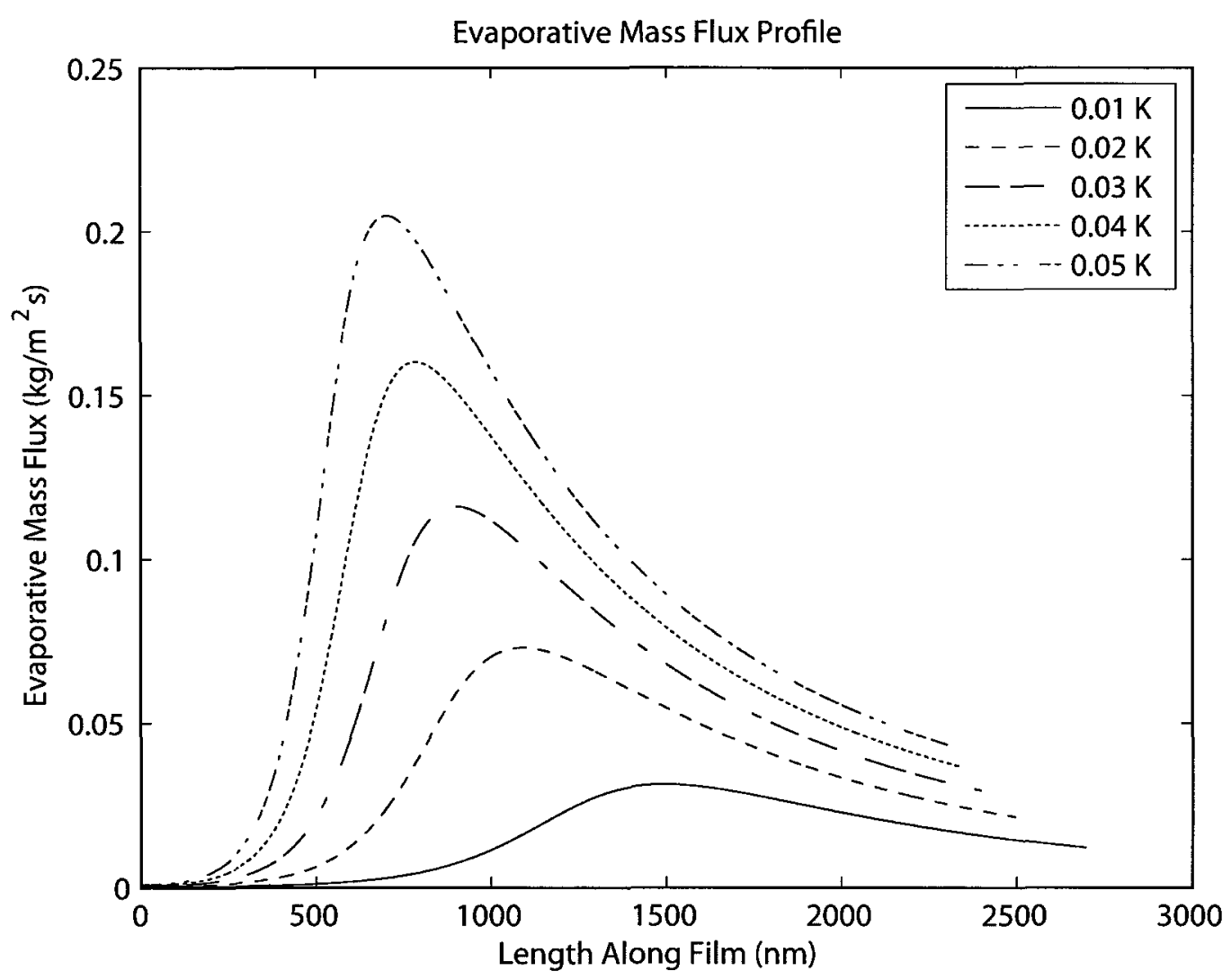

Figure 60: Superheat effects on evaporative mass flux

The evaporative characteristics of the thin film region as a function of applied superheat are given in Figure 60. As expected, the evaporative mass flux increases with the increase in applied superheat. For the starting region of the thin film, the evaporative mass flux is non-existent, thus satisfying the conditions of the adsorbed region.

This trend of increased capacity to evaporate is once again confirmed in the works of Wee et al. [9]. Of the differences in results, it is important to note that a jump in evaporative mass flux is found at the start of the thin film region as plotted by Wee et al. [9]. This jump is not present in the results shown above in Figure 60, lending credence to the model developed here. 
With each step increment in applied superheat the, evaporative mass flux profile contains all those below it. This attribute of the thin film is important as it ensures that for a high superheat, a greater mass flux can be attained.

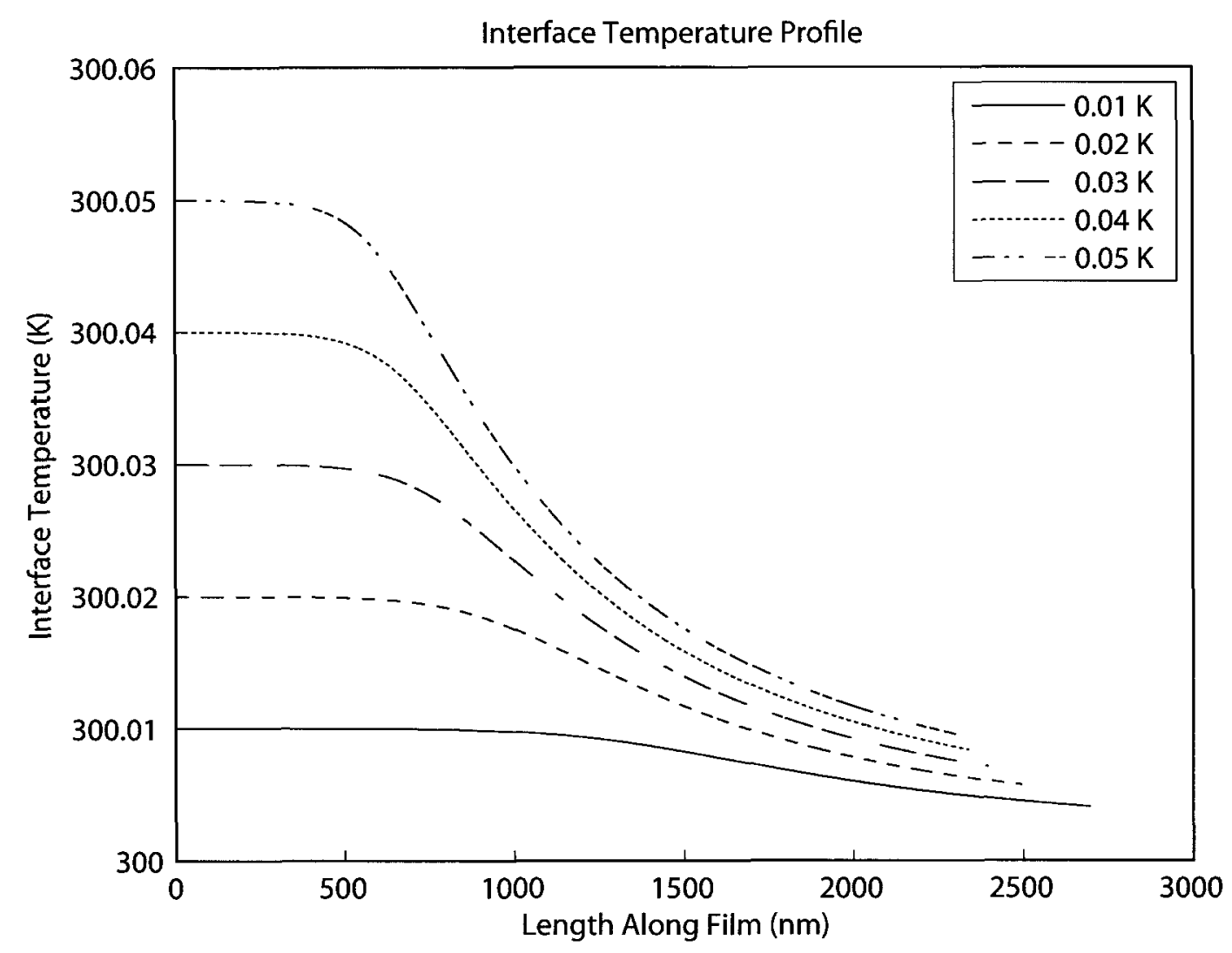

Figure 61: Superheat effects on interface temperature

Last to be reviewed is the variance of the interface temperature, as provided above in Figure 61. The temperature profiles all start at their respective applied superheats. As the temperature profiles progress along the length of the thin film, they tend to collect near the same region. 


\section{Chapter 6}

\section{Conclusions and Recommendations}

\subsection{Conclusions}

The study of thin film heat transfer is of interest to researchers and engineers, as it provides an effective and efficient means of transporting heat. The objective of this research was to formulate a mathematical model to represent the physical characteristics of the thin film, and its evaporative properties. The results of the model were selected so as to provide much needed information as to the behavioural characteristics for a variety of conditions.

To this end, a mathematical model was successfully developed, from the basic equations of fluid mechanics, thermodynamics and physics. The resulting model was that of a third order differential equation, subject to boundary conditions and initial conditions. The model developed includes the interfacial temperature gradient to ensure that any Marangoni effects would not be neglected, as well a wall slip condition was permitted in the Navier-Stokes equations.

The solving of the governing differential equation was done in MATLAB, with the use of Runge-Kutta solver. The program script was written so as to permit user defined geometry, operating fluid and physical conditions. This input and control capability allowed for the manipulation of the situations performed so as to capture 
the natural trends and tendencies of the differential equation. Thus the numerical model coding was used to produce results for a basic solution, solution uniqueness test, perturbation sensitivities, Marangoni effects, wall slip, different operating fluids, channel widths and applied superheats. The concluding remarks for each of the simulations performed are summarized below.

1. The first solution set obtained was for that of a simple case so as to determine if the solutions were reasonable. The thin film thickness was confirmed to monotonically increase from the adsorbed region through to the bulk meniscus region. The far field curvature was found to reach a constant value and was searched out for a curvature of $1 / r$, for the Young-Laplace solution. Of the data collected, it is possible to conclude that the thin film portion of the meniscus is dominated by the disjoining and capillary pressures. As the pressures switch from disjoining in the adsorbed region to capillary in the bulk region, the liquid is not as restricted and thus evaporation is easier. This is why the evaporative mass flux peaks in this region, thus confirming that evaporation rates are greater in the thin film region, than that of the bulk meniscus.

2. The uniqueness study was conducted to determine if a unique solution for the differential equation existed. The results of the study prove that a solution to the governing differential equation is not unique, but depend in part on the initial conditions and applied perturbations. The trends observed in the solutions to the differential equation, indicate that the thin film characteristics are simply shifted as a result of changing the perturbations applied to the adsorbed film thickness and the film slope and curvature. Thus it can be concluded that the thin film length increases as the initial perturbations approach zero, with no effect on the shape of the profiles. 
3. The second study performed was that of a parametric study of the perturbations applied to the initial conditions. The perturbations were studied independently over a range of values so as to determine how the results were affected. The results of the study indicated that the perturbations applied to the thin film thickness and slope were sensitive, while the perturbation applied to the second derivative was insensitive. The perturbations of $\epsilon_{1}$ and $\epsilon_{2}$, were observed to have similar sensitivities, with the results shifting the solutions in opposite directions with respect to the perturbation increase.

4. The interfacial temperature gradient term carried through the derivation, was to represent the Marangoni effects which may arise in the case of an evaporating thin film. The investigation into the temperature gradient revealed that for low applied superheats, the differences in the thin film formation were small. Thus, it can be understood, that for sufficiently low superheats, the interfacial temperature gradient can take a value of zero. This is ill advised, as differences in the simulation conditions can give rise to complications. Thus, the temperature gradient should be retained so as to remove any inaccuracies.

5. The potential for slip to occur between the thin film and the channel wall was studied. The results of the study prove that a slip condition at the wall can increase thin film length with little effect on the evaporative profiles. These results were observed to be similar to those of the uniqueness study.

6. For the case regarding the study of different fluids, two non-polar fluids were accurately modeled: namely pentane and octane. The results of the simulations clearly demonstrate how the operating fluid can affect the heat transfer characteristics of an evaporating thin film. Of the simulations conducted, it can be concluded that a difference in fluid can significantly affect the results. 
7. The geometry of the channel which held the evaporating thin film was successfully modeled. This simulation set was able to provide insight into the geometric effects on thin film evaporation. The results of this study show that an increase in channel width increases both the thin film length and the evaporative capabilities. It also shows that the increase in evaporation with channel width will reach a limit as dictated by the approach to a planar surface in the bulk region of the meniscus. In conclusion, the increase in channel width will asymptotically approach a limit for the evaporative mass flux and thin film length.

8. The last case studied was for that of different applied superheats. Of the simulations conducted, a trend of decreasing thin film length was observed with increasing superheat. The consequences of which, resulted in a curvature overshoot and significant increases in the evaporative mass flux. Thus it can be concluded, that with increasing superheat, the evaporation within the thin film increases with the curvature overshoot.

In conclusion, a mathematical model for an evaporating thin film was successfully developed and numerically solved. The model developed here, as demonstrated, proved to be robust and capable reproducing the main features of an evaporating thin film. 


\subsection{Recommendations}

The work completed in this thesis has demonstrated that the numerical model developed appropriately represents the formation of an evaporating thin film. This model is however limited in some aspects of applications and simplifying assumptions, and lack of experimental validation. Thus the model can be further advanced, through the removal of some of the assumptions and comparison to experimental data.

First is the experimental data requirement for validation of the numerical model. This aspect of thin film research is in short supply, with only a couple of experimental investigations completed. Of the data collected, the resolution of the measurements taken is near the thin film length scale, yet still too large for high superheat cases. Thus, an experimental investigation is needed with sufficient resolution so as to capture some of the characteristics such as the curvature overshoot. This would provide insight into the potential short comings of the numerical model.

As for the numerical model itself, a more expansive study of the applied superheats would be desired. The applied superheat could be expanded to that of several Kelvin. If higher superheats are to be studied, the temperature gradient term must remain in the derivation so as to ensure the Marangoni effects are not neglected.

For the cases where Marangoni vortices may be expected, the Navier-Stokes equations will need to be reconsidered, as the thin film will have flow transverse to the channel wall. This change in fluid flow will affect the entire derivation, requiring the transitioning from a one dimensional fluid flow to that of at least a two dimensional flow. As well, the convective heat transfer within the thin film will have an increasing effect on the thin film behaviour. 
Another simplification that can be removed would be the constant vapour pressure field. With the removal of this condition, both sides of the interface would require simultaneous solution of two sets Navier-Stokes equations. The first set of equations would be the same as those used above with the change in a couple of boundary conditions so as to ensure interaction between the vapour and liquid. The resulting model would provide a more consistent description of the thin film and possibly that of the bulk region.

With regards to the fluids used in the simulations, they were that of a nonpolar nature. This specifier restricts the use of common polar fluids such as water and ammonia. This can be addressed by the modification of the disjoining pressure equation so as to compensate for the difference in intermolecular forces. To achieve this however, two different mathematical models are required, with each retaining a piece of the same operational code developed in this work. 


\section{List of References}

[1] C. Sodtke, J. Kern, N. Schweizer, and P. Stephan, "High resolution measurements of wall temperature distribution underneath a single vapour bubble under low gravity conditions," International Journal of Heat and Mass Transfer, vol. 49, pp. 1100-1106, 2006.

[2] S. DasGupta, I. Y. Kim, and P. C. Wayner Jr., "Use of the kelvin-clapeyron equation to model an evaporating curved microfilm," Journal of Heat Transfer, vol. 116, pp. 1007-1015, 1994.

[3] J. J. Zhao, Y. Y. Duan, X. D. Wang, and B. X. Wang, "Effects of superheat and temperature-dependent thermophysical properties on evaporating thin liquid films in microchannels," International Journal of Heat and Mass Transfer, vol. 54, pp. 1259-1267, 2011.

[4] C. Sodtke and P. Stephan, "Spray cooling on micro structured surfaces," International Journal of Heat and Mass Transfer, vol. 50, pp. 4089-4097, 2007.

[5] H. Wang, S. V. Garimella, and J. Y. Murthy, "Characteristics of an evaporating thin film in a microchannel," International Journal of Heat and Mass Transfer, vol. 50, pp. 3933 - 3942, 2007.

[6] P. C. Wayner Jr., "Mechanical and thermal effects in the forced spreading of a liquid film with a finite contact angle," Colloids and Surfaces A: Physicochemical and Engineering Aspects, vol. 89, pp. 89-95, 1994.

[7] B. Derjaguin, N. Churaev, and V. Muller, Surface Forces. New York: Consultants Bureau, 1987.

[8] C. Höhmann and P. Stephan, "Microscale temperature measurement at an evaporating liquid meniscus," Experimental Thermal and Fluid Science, vol. 26, pp. 157-162, 2002. 
[9] S. K. Wee, K. D. Kihm, and K. P. Hallinan, "Effects of the liquid polarity and the wall slip on the heat and mass transport characteristics of the micro-scale evaporating transition film," International Journal of Heat and Mass Transfer, vol. 48, pp. 265-278, 2005.

[10] K. Stephan, "Influence of dispersion forces on phase equilibria between thin liquid films and their vapour," International Journal of Heat and Mass Transfer, vol. 45, pp. 4715-4725, 2002.

[11] W. Qu, T. Ma, J. Miao, and J. Wang, "Effects of radius and heat transfer on the profile of evaporating thin liquid film and meniscus in capillary tubes," International Journal of Heat and Mass Transfer, vol. 45, pp. 1879-1887, 2002.

[12] H. Wang, S. V. Garimella, and J. Y. Murthy, "An analytical solution for the total heat transfer in the thin-film region of an evaporating meniscus," International Journal of Heat and Mass Transfer, vol. 51, pp. 6317-6322, 2008.

[13] P. C. Wayner Jr., Y. Kao, and L. LaCroix, "The interline heat-transfer coefficient of an evaporating wetting film," International Journal of Heat and Mass Transfer, vol. 19, pp. 487-492, 1976.

[14] K. Park and K. S. Lee, "Flow and heat transfer characteristics of the evaporating extended meniscus in a micro-capillary channel," International Journal of Heat and Mass Transfer, vol. 46, pp. 4587-4594, 2003.

[15] K. Park, K. J. Noh, and K. S. Lee, "Transport phenomena in the thin-film region of a micro-channel," International Journal of Heat and Mass Transfer, vol. 46, pp. 2381-2388, 2003.

[16] S. S. Panchamgam, A. Chatterjee, J. L. Plawsky, and P. C. Wayner Jr., "Comprehensive experimental and theoretical study of fluid flow and heat transfer in a microscopic evaporating meniscus in a miniature heat exchanger," International Journal of Heat and Mass Transfer, vol. 51, pp. 5368-5379, 2008.

[17] P. C. Wayner Jr., "Intermolecular forces in phase-change heat transfer: 1998 kern award review," AIChE Journal, vol. 45, pp. 2055-2068, 1999.

[18] M. Potash Jr. and P. C. Wayner Jr., "Evaporation from a two-dimensional extended mensicus," International Journal of Heat and Mass Transfer, vol. 15, pp. 1851-1863, 1972. 
[19] S. DasGupta, J. A. Schonberg, I. Y. Kim, and P. C. Wayner Jr., "Use of the augmented Young-Laplace equation to model equilibrium and evaporating extended mensicus," Journal of Colloid and Interface Science, vol. 157, pp. 332-342, 1993.

[20] Q. He and K. P. Hallinan, "A new particle image velocimetry technique for threedimensional full field fluid flow measurement in evaporating films," Experimental Thermal and Fluid Science, vol. 17, pp. 230-237, 1998.

[21] J. A. Schonberg, S. DasGupta, and P. C. Wayner Jr., "An augmented YoungLaplace model of an evaporating meniscus in a microchannel with high heat flux," Experimental Thermal and Fluid Science, vol. 10, pp. 163-170, 2011.

[22] H. Wang, J. Y. Murthy, and S. V. Garimella, "Transport from a volatile meniscus inside an open microtube," International Journal of Heat and Mass Transfer, vol. 51, pp. 3007-3017, 2008.

[23] S. C. Maroo and J. N. Chung, "heat transfer characteristics and pressure variation in a nanoscale evaporating meniscus," International Journal of Heat and Mass Transfer, vol. 53, pp. 3335-3345, 2010.

[24] C. Buffone, K. Sefiane, and J. R. E. Christy, "Experimental investigation of the hydrodynamics and stability of an evaporating wetting film placed in a temperature gradient," Applied Thermal Engineering, vol. 24, pp. 1157-1170, 2004.

[25] T. Kaya and J. Goldak, "Numerical analysis of heat and mass transfer in the capillary structure of a loop heat pipe," International Journal of Heat and Mass Transfer, vol. 49, pp. 3211 - 3220, 2006.

[26] T. J. Lu, "Thermal management of high power electronics with phase change cooling," International Journal of Heat and Mass Transfer, vol. 43, pp. 22452256,2000 .

[27] H. J. Butt and M. Kappl, Surface and Interfacial Forces. Federal Republic of Germany: Wiley-VCH Verlag GmbH and Co. KGaA, Weinheim, 2010.

[28] H. C. Hamaker, "The London-van der Waals attraction between spherical particles," Physica, vol. 4, pp. 1058-1072, 1937.

[29] R. H. French, R. M. Cannon, L. K. DeNoyer, and Y. M. Chiang, "Full spectral calculation of non-retarded Hamaker constants for ceramic systems from interband transition strengths," Solid State Ionics, vol. 75, pp. 13-33, 1995. 
[30] P. C. Wayner Jr., "The effect of interfacial mass transport on flow in thin liquid films," Colloids and Surfaces, vol. 52, pp. 71-84, 1991.

[31] P. C. Wayner Jr., "Nucleation, growth and surface movement of a condensing sessile droplet," Colloids and Sufraces A: Physicochemical and Engineering Aspects, vol. 206, pp. 157-165, 2002.

[32] P. C. Stephan and C. A. Busse, "Analysis of the heat transfer coefficient of grooved heat pipe evaporator walls," International Journal of Heat and Mass Transfer, vol. 35, pp. 383-391, 1992.

[33] R. Ranjan, J. Y. Murthy, and S. V. Garimella, "A microscale model for thinfilm evaporating in capillary wick structures," International Journal of Heat and Mass Transfer, vol. 54, pp. 169-179, 2011.

[34] R. Ranjan, J. Y. Murthy, S. V. Garimella, and U. Vadakkan, "A numerical model for transport in flat heat pipes considering wick microstructure effects," International Journal of Heat and Mass Transfer, vol. 54, pp. 153-168, 2011.

[35] S. S. Panchamgam, J. L. Plawsky, and P. C. Wayner Jr., "Microscale heat transfer in an evaporating moving extended meniscus," Experimental Thermal and Fluid Science, vol. 30, pp. 745-754, 2006.

[36] F. M. White, Fluid Mechanics. McGraw Hill Higher Education, 6th ed., 2008.

[37] J. P. Holman, Heat Transfer. McGraw Hill, 10th international edition ed., 2010.

[38] K. A. Zachariasse, T. von der Haar, A. Hebecker, U. Leinhos, and W. Kühnle, "Intramolecular charge transfer in amino-benzonitriles: Requirements for dual flourescence," Pure and Applied Chemistry, vol. 65, pp. 1745-1750, 1993.

[39] E. A. Costner, B. K. Long, C. Navar, S. Jockusch, X. Lei, P. Zimmerman, A. Campion, N. J. Turro, and C. G. Willson, "Fundamental optical properties of linear and cyclic alkanes: Absorbance and index of refraction," Journal of Physical Chemistry, pp. 9337-9347, 2009.

[40] A. Catenaccio, Y. Daruich, and C. Magallanes, "Temperature dependence of the permittivity of water," Chemical Physics Letters, vol. 367, pp. 669-671, 2003.

[41] A. Mingarelli, Calculus. The Nolan Company, 1st edition ed., 2001.

[42] J. R. Dormand and P. J. Prince, "A family of embedded Runge-Kutta formulae," Journal of Computational and Applied Mathematics, vol. 6, pp. 19-26, 1980. 


\section{Appendix A}

\section{Derivation of Thin Film Model}

The development of a mathematical model for the thin film portion of an evaporating meniscus uses the following physical representation.

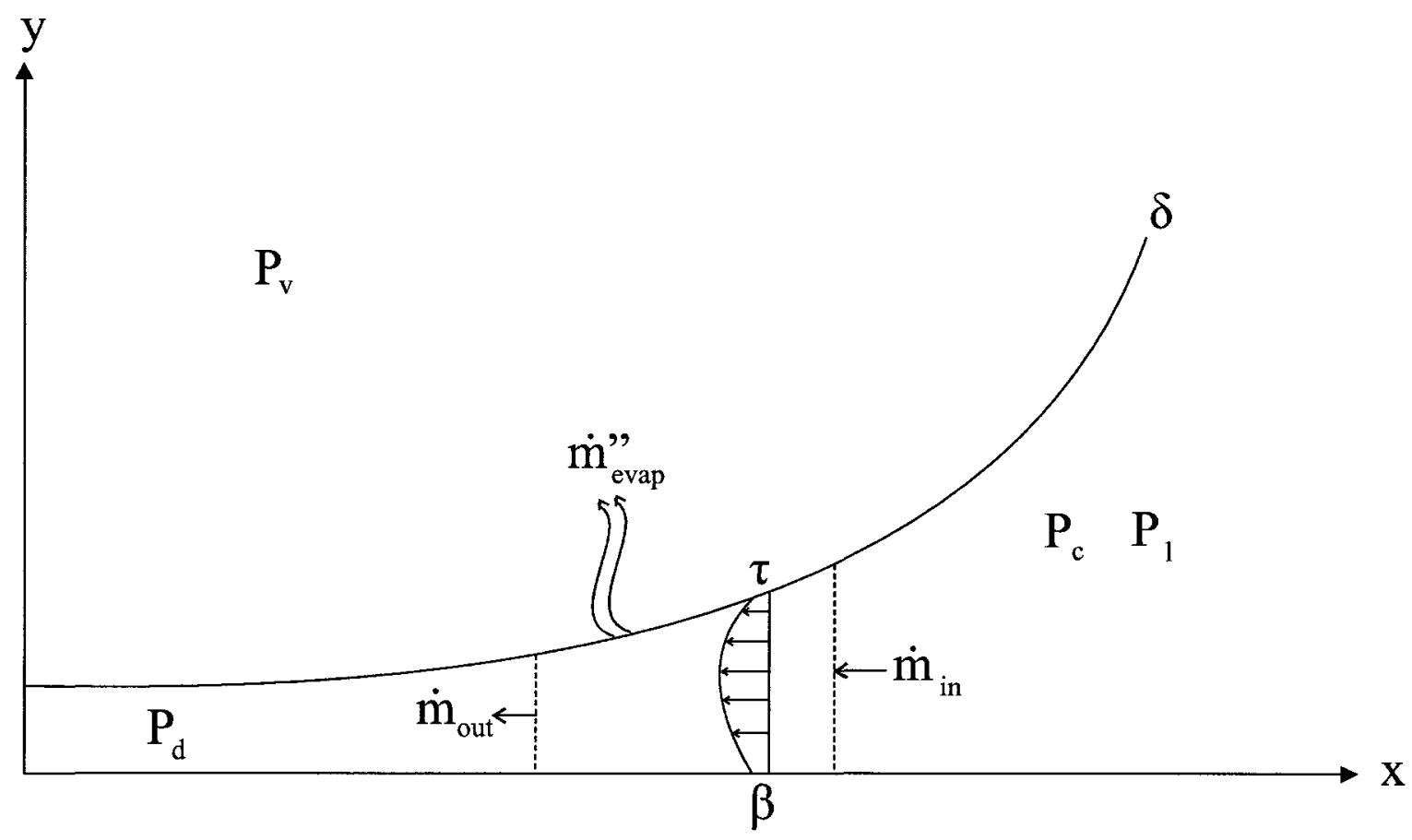

Figure 62: Thin film portion of a meniscus 
The coordinate system is set such that the origin is placed adjacent to the end of adsorbed region and the start of the evaporating thin film. The model employs the following simplifying assumptions:

- no liquid flow in y or z directions

- no thermal conduction through the liquid in the $\mathrm{x}$ and $\mathrm{z}$ directions

- vapour pressure is uniform and constant

- steady state

Solving the force balance on the thin liquid film,

$$
P_{v}=P_{l}+P_{c}+P_{d}
$$

where,

$$
P_{d}=\frac{A}{\delta^{3}}
$$

$$
P_{c}=\sigma \kappa
$$

$\kappa$ is the local curvature, defined by:

$$
\kappa=\frac{\delta^{\prime \prime}}{\left(1+\delta^{\prime 2}\right)^{3 / 2}}
$$

let,

$$
\alpha=1+\delta^{\prime 2}
$$

Combining all of the pressure terms in Equation (65),

$$
P_{v}=P_{l}+\sigma \kappa+\frac{A}{\delta^{3}}
$$


In taking the derivative of the force balance, Equation (65), with respect to the film length,

$$
\frac{d P_{v}}{d x}=\frac{d P_{l}}{d x}+\frac{d P_{c}}{d x}+\frac{d P_{d}}{d x}
$$

The following outlines the process of obtaining a third order ordinary differential equation:

$$
\frac{d P_{v}}{d x}=\frac{d P_{l}}{d x}+\sigma^{\prime} \kappa+\sigma \kappa^{\prime}-\frac{3 A}{\delta^{4}} \delta^{\prime}
$$

As the vapour pressure is assumed uniform and constant, the gradient must then be zero. In solving for the third order terms, the curvature gradient is,

$$
\kappa^{\prime}=\frac{\delta^{\prime \prime \prime}}{\alpha^{3 / 2}}-\frac{3 \delta^{\prime} \delta^{\prime 2}}{\alpha^{5 / 2}}
$$

Now to rearrange and solve for the liquid pressure gradient term.

$$
\begin{aligned}
-\frac{d P_{l}}{d x} & =\sigma^{\prime} \kappa+\sigma \kappa^{\prime}-\frac{3 A}{\delta^{4}} \delta^{\prime} \\
& =\sigma^{\prime} \frac{\delta^{\prime \prime}}{\alpha^{3 / 2}}+\sigma\left(\frac{\delta^{\prime \prime \prime}}{\alpha^{3 / 2}}-\frac{3 \delta^{\prime} \delta^{\prime \prime 2}}{\alpha^{5 / 2}}\right)-\frac{3 A}{\delta^{4}} \delta^{\prime}
\end{aligned}
$$

Rearranging for the third order film thickness term.

$$
\begin{aligned}
\delta^{\prime \prime \prime} & =\frac{3 \delta^{\prime} \delta^{\prime 2}}{\alpha}+\frac{3 A}{\delta^{4}}\left(\frac{\alpha^{3 / 2}}{\sigma}\right) \delta^{\prime}-\frac{\sigma^{\prime}}{\sigma} \delta^{\prime \prime}-\frac{d P_{l}}{d x}\left(\frac{\alpha^{3 / 2}}{\sigma}\right) \\
& =\frac{3 \delta^{\prime} \delta^{\prime 2}}{\alpha}-\frac{\sigma^{\prime}}{\sigma} \delta^{\prime \prime}+\frac{\alpha^{3 / 2}}{\sigma}\left(\frac{3 A}{\delta^{4}} \delta^{\prime}-\frac{d P_{l}}{d x}\right)
\end{aligned}
$$

Where,

$$
\begin{gathered}
\sigma=a+b T_{l v} \\
\sigma^{\prime}=b \frac{d T_{l v}}{d x}
\end{gathered}
$$


Thus,

$$
\delta^{\prime \prime \prime}=\frac{3 \delta^{\prime} \delta^{\prime 2}}{\alpha}+\frac{\alpha^{3 / 2}}{\sigma}\left(\frac{3 A}{\delta^{4}} \delta^{\prime}-\frac{d P_{l}}{d x}\right)-\frac{b}{\sigma}\left(\frac{d T_{l v}}{d x}\right) \delta^{\prime \prime}
$$

Equation (78) is a third order ordinary differential equation which describes the profile of the evaporating thin film. The equation requires knowledge of the liquid pressure gradient and the interfacial temperature gradient. Using Navier-Stokes, the liquid pressure gradient can be related to the velocity field in the thin film. Using the Cartesian form of the Navier-Stokes equations, two of the equations can be discarded as the assumptions prohibit flow in the $y$ and $z$ directions. Thus the flow profile in the $x$ direction is given by,

$$
\rho\left(\frac{d u}{d t}+u \frac{d u}{d x}+v \frac{d u}{d y}+w \frac{d u}{d z}\right)=-\frac{d P_{l}}{d x}+\mu\left(\frac{d^{2} u}{d x^{2}}+\frac{d^{2} u}{d y^{2}}+\frac{d^{2} u}{d z^{2}}\right)+\rho g_{x}
$$

The liquid pressure gradient is obtained from Equation (79) with the application of associated boundary conditions. Thus the liquid pressure gradient is,

$$
\frac{d P_{l}}{d x}=\mu \frac{d^{2} u}{d y^{2}}
$$

Integrating Equation (80), we obtain the liquid pressure gradient as a function of thin film fluid velocity.

$$
\frac{1}{\mu} \int \frac{d P_{l}}{d x} d y=\int \frac{d^{2} u}{d y^{2}} d y
$$

Thus,

$$
\frac{1}{\mu} \frac{d P_{l}}{d x}\left(y+C_{1}\right)=\frac{d u}{d y}
$$

Integrating Equation (82),

$$
\frac{1}{\mu} \frac{d P_{l}}{d x} \int\left(y+C_{1}\right) d y=\int \frac{d u}{d y} d y
$$


Integrating once more to obtain the velocity profile,

$$
\frac{1}{\mu} \frac{d P_{l}}{d x}\left(\frac{y^{2}}{2}+C_{1} y+C_{2}\right)=u
$$

The boundary conditions associated with the flow are:

At the wall: $y=0$

$$
u=u_{s}=-\beta \frac{d u}{d y}
$$

At the liquid vapour interface: $y=\delta$

$$
\tau=\sigma^{\prime}=\mu \frac{d u}{d y}
$$

Applying boundary condition (85) to Equation (84),

$$
C_{2}=\mu u_{s} \frac{d x}{d P_{l}}
$$

Applying boundary condition (86) to Equation (82)

$$
C_{1}=\sigma^{\prime} \frac{d x}{d P_{l}}-\delta
$$

Combining Equations (84), (87) and (88), to obtain a relationship for the thin film velocity.

$$
\begin{aligned}
u & =\frac{1}{\mu} \frac{d P_{l}}{d x}\left(\frac{y^{2}}{2}+y\left(\sigma^{\prime} \frac{d x}{d P_{l}}-\delta\right)+\mu u_{s} \frac{d x}{d P_{l}}\right) \\
& =\frac{1}{\mu} \frac{d P_{l}}{d x} \frac{y^{2}}{2}+\frac{\sigma^{\prime}}{\mu} y-y \frac{\delta}{\mu} \frac{d P_{l}}{d x}+u_{s} \\
& =\frac{1}{\mu} \frac{d P_{l}}{d x}\left(\frac{y^{2}}{2}-y \delta\right)+\frac{\sigma^{\prime}}{\mu} y+u_{s}
\end{aligned}
$$


We still need a relationship for $u_{s}$, which can be obtained by integrating Equation (80) with definite boundary conditions (85) and (86).

$$
\int_{0}^{\delta} \frac{d P_{l}}{d x} d y=\mu \int_{0}^{\delta} \frac{d^{2} u}{d y^{2}} d y
$$

Thus the wall slip velocity is defined as,

$$
u_{s}=\frac{\beta}{\mu}\left(\frac{d P_{l}}{d x} \delta-\sigma^{\prime}\right)
$$

Combing Equations (91) and (89) and collecting terms, we obtain the thin film velocity profile.

$$
\begin{aligned}
u & =\frac{1}{\mu} \frac{d P_{l}}{d x}\left(\frac{y^{2}}{2}-y \delta\right)+\frac{\sigma^{\prime}}{\mu} y+\frac{\beta}{\mu}\left(\frac{d P_{l}}{d x} \delta-\sigma^{\prime}\right) \\
& =\frac{1}{\mu} \frac{d P_{l}}{d x}\left(\frac{y^{2}}{2}-y \delta+\beta \delta\right)+\frac{\sigma^{\prime}}{\mu}(y-\beta) \\
& =\frac{1}{\mu} \frac{d P_{l}}{d x}\left(\frac{y^{2}}{2}-\delta(y-\beta)\right)+\frac{\sigma^{\prime}}{\mu}(y-\beta)
\end{aligned}
$$

Yet, the surface tension gradient is a function of the interfacial temperature gradient as described by Equation (77). This now provides a thin film fluid velocity profile,

$$
u=\frac{1}{\mu} \frac{d P_{l}}{d x}\left(\frac{y^{2}}{2}-\delta(y-\beta)\right)+\frac{b}{\mu} \frac{d T_{l v}}{d x}(y-\beta)
$$

Next is to solve for the mass flow rate in the thin film using the continuity equation. Note, the cross sectional area is defined between the interface and the wall and is taken to be perpendicular to the $x$ axis. The area is defined as $A=d y d z$, and $d z$ is taken to be unity.

$$
\dot{m}=\rho A u
$$


Integrating Equation (94) with respect to $y$,

$$
\begin{aligned}
\dot{m} & =\int_{0}^{\delta} \rho u d y \\
& =\int_{0}^{\delta} \rho\left[\frac{1}{\mu} \frac{d P_{l}}{d x}\left(\frac{y^{2}}{2}-\delta(y-\beta)\right)+\frac{b}{\mu} \frac{d T_{l v}}{d x}(y-\beta)\right] d y \\
& =\frac{1}{\nu} \frac{d P_{l}}{d x} \int_{0}^{\delta}\left[\frac{y^{2}}{2}-\delta(y-\beta)\right] d y+\frac{b}{\nu} \frac{d T_{l v}}{d x} \int_{0}^{\delta}[y-\beta] d y \\
& =\frac{1}{\nu} \frac{d P_{l}}{d x}\left[\frac{y^{3}}{6}-\delta\left(\frac{y^{2}}{2}-\beta y\right)\right]_{0}^{\delta}+\frac{b}{\nu} \frac{d T_{l v}}{d x}\left[\frac{y^{2}}{2}-\beta y\right]_{0}^{\delta} \\
& =\frac{1}{\nu} \frac{d P_{l}}{d x}\left[\frac{\delta^{3}}{6}-\frac{\delta^{3}}{2}+\beta \delta^{2}\right]+\frac{b}{\nu} \frac{d T_{l v}}{d x}\left[\frac{\delta^{2}}{2}-\beta \delta\right] \\
& =\frac{1}{\nu} \frac{d P_{l}}{d x}\left[-\frac{\delta^{3}}{3}+\beta \delta^{2}\right]+\frac{b}{\nu} \frac{d T_{l v}}{d x}\left[\frac{\delta^{2}}{2}-\beta \delta\right]
\end{aligned}
$$

Next is to accommodate for the heat transfer via evaporation. This can be accomplished by using the energy equation assuming,

- No thermal conduction in the $\mathrm{x}$ or $\mathrm{z}$ directions

- No heat generation within the film layer

- No transient response or effects

$$
\frac{\partial}{\partial x}\left(k_{l} \frac{\partial T}{\partial x}\right)+\frac{\partial}{\partial y}\left(k_{l} \frac{\partial T}{\partial y}\right)+\frac{\partial}{\partial z}\left(k_{l} \frac{\partial T}{\partial z}\right)+\dot{q}=\rho c_{p} \frac{\partial T}{\partial t}
$$

Applying the above stated assumptions,

$$
\frac{\partial}{\partial y}\left(k_{l} \frac{\partial T}{\partial y}\right)=0
$$

The boundary conditions to be associated with Equation (97) are: At the wall, $y=0$ :

$$
T=T_{w}
$$


At the liquid vapour interface, $y=\delta$ :

$$
-k_{l} \frac{\partial T}{\partial y}=\dot{m}_{e v a p}^{\prime \prime} h_{f g}
$$

Applying boundary conditions (98) and (99) to the energy equation (97) and integrating with respect to $y$, we can obtain the evaporative mass flux.

$$
\begin{aligned}
\int \frac{\partial}{\partial y}\left(k_{l} \frac{\partial T}{\partial y}\right) d y & =0 \\
k_{l} \frac{\partial T}{\partial y} & =C_{3}
\end{aligned}
$$

Solving for the constant of integration in Equation (100) through the application of boundary condition (99).

$$
\begin{aligned}
k_{l} \frac{\partial T}{\partial y} & =-\dot{m}_{\text {evap }}^{\prime \prime} h_{f g} \\
k_{l}\left(\frac{T_{l v}-T_{w}}{\delta-0}\right) & =-\dot{m}_{\text {evap }}^{\prime \prime} h_{f g} \\
-k_{l}\left(\frac{T_{w}-T_{l v}}{\delta}\right) & =-\dot{m}_{\text {evap }}^{\prime \prime} h_{f g} \\
\dot{m}_{\text {evap }}^{\prime \prime} & =\frac{k_{l}\left(T_{w}-T_{l v}\right)}{\delta h_{f g}}
\end{aligned}
$$

Equation (101) is representative of the evaporative mass flux at the interface of the thin film. Of particular importance is the condition of steady state evaporation. For the meniscus to be stable, the mass flow into the thin film region must be balanced by the evaporative mass flux. Thus a relation for the mass flow balance can be derived as follows:

$$
\dot{m}_{i n}=\rho A u_{i n}
$$


Due to the direction of the fluid flow in the thin film region, the velocity profile in relation to the chosen coordinate system is to be taken as negative. And for the evaporative mass flux, the area is to be taken to be $d x$ and unity in $z$. This results in,

$$
\begin{aligned}
\left(-\dot{m}_{\imath n}\right)-\left(-\dot{m}_{\text {out }}\right) & =\dot{m}_{\text {evap }}^{\prime \prime} d x d z \\
\dot{m}_{\text {evap }}^{\prime \prime} & =\frac{-\dot{m}_{\imath n}+\dot{m}_{\text {out }}}{x_{2}-x_{1}} \\
-\dot{m}_{\text {evap }}^{\prime \prime} & =\frac{d \dot{m}}{d x}
\end{aligned}
$$

Equating Equations (103) and (101), and integrating results in a relation for the mass flow in the thin film as a function of thin film length.

$$
\begin{aligned}
-\frac{d \dot{m}}{d x} & =\frac{k_{l}\left(T_{w}-T_{l v}\right)}{\delta h_{f g}} \\
-\int d \dot{m} & =\int_{0}^{x} \frac{k_{l}\left(T_{w}-T_{l v}\right)}{\delta h_{f g}} d x \\
\dot{m} & =-\int_{0}^{x} \frac{k_{l}\left(T_{w}-T_{l v}\right)}{\delta h_{f g}} d x
\end{aligned}
$$

Equating the evaporative mass flux as described by Equations (95) and (104). This will leads to a relation for the liquid pressure gradient and the interfacial temperature gradient.

$$
\begin{aligned}
-\int_{0}^{x} \frac{k_{l}\left(T_{w}-T_{l v}\right)}{\delta h_{f g}} d x & =\frac{1}{\nu} \frac{d P_{l}}{d x}\left(-\frac{\delta^{3}}{3}+\beta \delta^{2}\right)+\frac{b}{\nu} \frac{d T_{l v}}{d x}\left(\frac{\delta^{2}}{2}-\beta \delta\right) \\
-\int_{0}^{x} \frac{k_{l}\left(T_{w}-T_{l v}\right)}{\delta h_{f g}} d x & =-\frac{1}{\nu} \frac{d P_{l}}{d x}\left(\frac{\delta^{3}}{3}-\beta \delta^{2}\right)+\frac{b}{\nu} \frac{d T_{l v}}{d x}\left(\frac{\delta^{2}}{2}-\beta \delta\right) \\
\frac{1}{\nu} \frac{d P_{l}}{d x}\left(\frac{\delta^{3}}{3}-\beta \delta^{2}\right) & =\frac{b}{\nu} \frac{d T_{l v}}{d x}\left(\frac{\delta^{2}}{2}+\beta \delta\right)+\int_{0}^{x} \frac{k_{l}\left(T_{w}-T_{l v}\right)}{\delta h_{f} g} d x \\
\frac{d P_{l}}{d x}\left(\frac{\delta^{3}}{3}-\beta \delta^{2}\right) & =b \frac{d T_{l v}}{d x}\left(\frac{\delta^{2}}{2}+\beta \delta\right)+\nu \int_{0}^{x} \frac{k_{l}\left(T_{w}-T_{l v}\right)}{\delta h_{f g}} d x
\end{aligned}
$$


Letting,

$$
\begin{aligned}
& C_{4}=\left(\frac{\delta^{3}}{3}-\beta \delta^{2}\right) \\
& C_{5}=\left(\frac{\delta^{2}}{2}-\beta \delta\right)
\end{aligned}
$$

Thus, the liquid pressure gradient is defined by,

$$
\frac{d P_{l}}{d x}=b \frac{C_{5}}{C_{4}} \frac{d T_{l v}}{d x}+\frac{\nu k_{l}}{C_{4} h_{f g}} \int_{0}^{x} \frac{T_{w}-T_{l v}}{\delta} d x
$$

We still need one more relation for the evaporative mass flux. Thus, the KelvinClapeyron model will provide the relation as a function of the interfacial temperature.

$$
\dot{m}_{\text {evap }}^{\prime \prime}=\frac{1}{h_{f g}}\left(h_{l v}^{c l}\left(T_{l v}-T_{v}\right)-h_{l v}^{k l}\left(P_{d}+P_{c}\right)\right)
$$

Given,

$$
\begin{gathered}
h_{l v}^{c l}=\eta\left(\frac{1}{T_{l v}}\right)^{3 / 2}\left(\frac{M h_{f g}}{T_{v}}\right) \\
h_{l v}^{k l}=\eta\left(\frac{1}{T_{l v}}\right)^{3 / 2} V_{l}
\end{gathered}
$$

Provided,

$$
\eta=\left(\frac{C^{2} M}{2 \pi R}\right)^{1 / 2}\left(\frac{P_{v} h_{f g}}{R}\right)
$$

Thus the Kelvin-Clapeyron evaporative mass flux model is then,

$$
\dot{m}_{\text {evap }}^{\prime \prime}=\frac{\eta}{h_{f g}}\left(\frac{1}{T_{l v}}\right)^{3 / 2}\left[\frac{M h_{f g}}{T_{v}}\left(T_{l v}-T_{v}\right)-V_{l}\left(P_{d}+P_{c}\right)\right]
$$


By equating the evaporative mass flux relations defined by Equations (101) and (113), a relation for the interfacial temperature can be obtained as follows:

$$
\begin{aligned}
\frac{\eta}{h_{f g}}\left(\frac{1}{T_{l v}}\right)^{3 / 2}\left[\frac{M h_{f g}}{T_{v}}\left(T_{l v}-T_{v}\right)-V_{l}\left(P_{d}+P_{c}\right)\right] & =\frac{k_{l}\left(T_{w}-T_{l v}\right)}{\delta h_{f g}} \\
\frac{M h_{f g}}{T_{v}}\left(T_{l v}-T_{v}\right)-V_{l}\left(P_{d}+P_{c}\right) & =\frac{k_{l}}{\delta \eta}\left(T_{w}-T_{l v}\right) T_{l v}^{3 / 2} \\
\frac{M h_{f g}}{T_{v}} T_{l v}-M h_{f g}-V_{l}\left(P_{d}+P_{c}\right) & =\frac{k_{l}}{\delta \eta} T_{w} T_{l v}^{3 / 2}-\frac{k_{l}}{\delta \eta} T_{l v}^{5 / 2} \\
\frac{k_{l}}{\delta \eta} T_{l v}^{5 / 2}-\frac{k_{l}}{\delta \eta} T_{w} T_{l v}^{3 / 2}+\frac{M h_{f g}}{T_{v}} T_{l v} & =M h_{f g}+V_{l}\left(P_{d}+P_{c}\right)
\end{aligned}
$$

Using the equations for capillary pressure (67), disjoining pressure (66) and surface tension (76), the interfacial temperature relation, Equation (114), can be further expanded. The expansion will yield an interfacial temperature relation as a function of fluid properties, film thickness and associated derivatives.

$$
\begin{aligned}
\frac{k_{l}}{\delta \eta} T_{l v}^{5 / 2}-\frac{k_{l}}{\delta \eta} T_{w} T_{l v}^{3 / 2}+\frac{M h_{f g}}{T_{v}} T_{l v} & =M h_{f g}+V_{l}\left(\frac{A}{\delta^{3}}+\left(a+b T_{l v}\right) \kappa\right) \\
\frac{k_{l}}{\delta \eta} T_{l v}^{5 / 2}-\frac{k_{l}}{\delta \eta} T_{w} T_{l v}^{3 / 2}+\frac{M h_{f g}}{T_{v}} T_{l v} & =M h_{f g}+V_{l}\left(\frac{A}{\delta^{3}}\right)+V_{l} a \kappa+V_{l} b T_{l v} \kappa \\
\frac{k_{l}}{\delta \eta} T_{l v}^{5 / 2}-\frac{k_{l}}{\delta \eta} T_{w} T_{l v}^{3 / 2}+\frac{M h_{f g}}{T_{v}} T_{l v}-V_{l} b T_{l v} \kappa & =M h_{f g}+V_{l}\left(\frac{A}{\delta^{3}}\right)+V_{l} a \kappa \\
\frac{k_{l}}{\delta \eta} T_{l v}^{5 / 2}-\frac{k_{l}}{\delta \eta} T_{w} T_{l v}^{3 / 2}+\frac{M h_{f g}}{T_{v}} T_{l v}-V_{l} b T_{l v} \kappa & =M h_{f g}+V_{l}\left(\frac{A}{\delta^{3}}+a \kappa\right)
\end{aligned}
$$

Now that the relation for the interface temperature is defined, the interfacial temperature gradient can be obtained, by differentiating Equation (115) with respect to $x$. To simplify the derivatives, each set of terms given in Equation (115), is differentiated below.

$$
\frac{d}{d x}\left(\frac{k_{l}}{\delta \eta} T_{l v}^{5 / 2}\right)=-\frac{k_{l}}{\delta^{2} \eta} \delta^{\prime} T_{l v}^{5 / 2}+\frac{k_{l}}{\delta \eta}\left(\frac{5}{2}\right) T_{l v}^{3 / 2} \frac{d T_{l v}}{d x}
$$




$$
\begin{gathered}
\frac{d}{d x}\left(\frac{k_{l}}{\delta \eta} T_{l v}^{3 / 2}\right) T_{w}=-\frac{k_{l}}{\delta^{2} \eta} \delta^{\prime} T_{w} T_{l v}^{3 / 2}+\frac{k_{l}}{\delta \eta}\left(\frac{3}{2}\right) T_{w} T_{l v}^{1 / 2} \frac{d T_{l v}}{d x} \\
\frac{d}{d x}\left(\frac{M h_{f g}}{T_{v}} T_{l v}\right)=\frac{M h_{f g}}{T_{v}} \frac{d T_{l v}}{d x} \\
\frac{d}{d x}\left(V_{l} b T_{l v} \kappa\right)=V_{l} b\left(\kappa^{\prime} T_{l v}+\kappa \frac{d T_{l v}}{d x}\right) \\
\frac{d}{d x}\left(M h_{f g}\right)=0 \\
\frac{d}{d x}\left(V_{l} \frac{A}{\delta^{3}}\right)=-\frac{3 A}{\delta^{4}} \delta^{\prime} V_{l} \\
\frac{d}{d x}\left(V_{l} a \kappa\right)=V_{l} a \kappa^{\prime}
\end{gathered}
$$

Combining the differentiated terms, Equation (116) through Equation (121), and rearranging to obtain the interfacial temperature gradient.

$$
\begin{aligned}
&-\frac{3 A}{\delta^{4}} \delta^{\prime} V_{l}+V_{l} a \kappa^{\prime}= {\left[-\frac{k_{l}}{\delta^{2} \eta} \delta^{\prime} T_{l v}^{5 / 2}+\frac{k_{l}}{\delta \eta}\left(\frac{5}{2}\right) T_{l v}^{3 / 2} \frac{d T_{l v}}{d x}\right]-} \\
& {\left[-\frac{k_{l}}{\delta^{2} \eta} \delta^{\prime} T_{l v}^{3 / 2}+\frac{k_{l}}{\delta \eta}\left(\frac{3}{2}\right) T_{l v}^{1 / 2} T_{w} \frac{d T_{l v}}{d x}\right]+} \\
& \frac{M h_{f g}}{T_{v}} \frac{d T_{l v}}{d x}-V_{l} b\left[\kappa^{\prime} T_{l v}+\kappa \frac{d T_{l v}}{d x}\right] \\
& \frac{d T_{l v}}{d x}\left[\frac{5 k_{l} T_{l v}^{3 / 2}}{2 \delta \eta}-\frac{3 k_{l} T_{w} T_{l v}^{1 / 2}}{2 \delta \eta}+\frac{M h_{f g}}{T_{v}}-V_{l} b \kappa\right]= \\
& V_{l}\left[a \kappa^{\prime}-\frac{3 A}{\delta^{4}} \delta^{\prime}\right]+\frac{k_{l}}{\delta^{2} \eta} \delta^{\prime} T_{l v}^{5 / 2}-\frac{k_{l}}{\delta^{2} \eta} \delta^{\prime} T_{w} T_{l v}^{3 / 2}+V_{l} b \kappa^{\prime} T_{l v}
\end{aligned}
$$




$$
\begin{gathered}
\frac{d T_{l v}}{d x}\left[\frac{k_{l} T_{v}\left(5 T_{l v}^{3 / 2}-3 T_{w} T_{l v}^{1 / 2}\right)+2 \delta \eta\left(M h_{f g}-V_{l} T_{v} b \kappa\right)}{2 \delta \eta T_{v}}\right]= \\
V_{l}[\kappa^{\prime} \underbrace{\left(a+b T_{l v}\right)}_{\sigma}-\frac{3 A}{\delta^{4}} \delta^{\prime}]+\frac{k_{l}}{\delta^{2} \eta} \delta^{\prime}\left(T_{l v}^{5 / 2}-T_{w} T_{l v}^{3 / 2}\right)
\end{gathered}
$$

$$
\begin{gathered}
\frac{d T_{l v}}{d x}\left[\frac{k_{l} T_{v}\left(5 T_{l v}^{3 / 2}-3 T_{w} T_{l v}^{1 / 2}\right)+2 \delta \eta\left(M h_{f g}-V_{l} T_{v} b \kappa\right)}{2 \delta \eta T_{v}}\right]= \\
V_{l}\left[\frac{\kappa^{\prime} \sigma \delta^{4}-3 A \delta^{\prime}}{\delta^{4}}\right]+\frac{k_{l} \delta^{\prime}\left(T_{l v}^{5 / 2}-T_{w} T_{l v}^{3 / 2}\right)}{\delta^{2} \eta}
\end{gathered}
$$

$$
\begin{gathered}
\frac{d T_{l v}}{d x}\left[\frac{k_{l} T_{v}\left(5 T_{l v}^{3 / 2}-3 T_{w} T_{l v}^{1 / 2}\right)+2 \delta \eta\left(M h_{f g}-V_{l} T_{v} b \kappa\right)}{2 \delta \eta T_{v}}\right]= \\
{\left[\frac{V_{l} \eta\left(\kappa^{\prime} \sigma \delta^{4}-3 A \delta^{\prime}\right)+k_{l} \delta^{2} \delta^{\prime}\left(T_{l v}^{5 / 2}-T_{w} T_{l v}^{3 / 2}\right)}{\delta^{4} \eta}\right]}
\end{gathered}
$$

$$
\frac{d T_{l v}}{d x}=\left(\frac{2 \delta \eta T_{v}}{\delta^{4} \eta}\right)\left[\frac{V_{l} \eta\left(\kappa^{\prime} \sigma \delta^{4}-3 A \delta^{\prime}\right)+k_{l} \delta^{2} \delta^{\prime}\left(T_{l v}^{5 / 2}-T_{w} T_{l v}^{3 / 2}\right)}{k_{l} T_{v}\left(5 T_{l v}^{3 / 2}-3 T_{w} T_{l v}^{1 / 2}\right)+2 \delta \eta\left(M h_{f g}-V_{l} T_{v} b \kappa\right)}\right]
$$

$$
\frac{d T_{l v}}{d x}=\left(\frac{2 T_{v}}{\delta^{3}}\right)\left[\frac{\delta^{\prime}\left[k_{l} \delta^{2}\left(T_{l v}^{5 / 2}-T_{w} T_{l v}^{3 / 2}\right)-3 A V_{l} \eta\right]+V_{l} \eta \sigma \delta^{4} \kappa^{\prime}}{k_{l} T_{v}\left(5 T_{l v}^{3 / 2}-3 T_{w} T_{l v}^{1 / 2}\right)+2 \delta \eta\left(M h_{f g}-V_{l} T_{v} b \kappa\right)}\right]
$$


Assigning variables to the portions of the denominator and numerator for the interfacial temperature gradient, Equation (129),

$$
\begin{gathered}
\chi=\delta^{\prime}\left[k_{l} \delta^{2}\left(T_{l v}^{5 / 2}-T_{w} T_{l v}^{3 / 2}\right)-3 A V_{l} \eta\right] \\
\omega=k_{l} T_{v}\left(5 T_{l v}^{3 / 2}-3 T_{w} T_{l v}^{1 / 2}\right)+2 \delta \eta\left(M h_{f g}-V_{l} T_{v} b \kappa\right)
\end{gathered}
$$

Thus the interfacial temperature gradient is described by,

$$
\frac{d T_{l v}}{d x}=\left(\frac{2 T_{v}}{\delta^{3}}\right)\left(\frac{\chi+V_{l} \eta \sigma \delta^{4} \kappa^{\prime}}{\omega}\right)
$$

We now have all of the unknown terms required for the solving of the third order ordinary differential Equation (78). The first step will be to combine Equations (78) and (108).

$$
\begin{gathered}
\delta^{\prime \prime \prime}=\frac{3 \delta^{\prime} \delta^{\prime \prime 2}}{\alpha}+\frac{\alpha^{3 / 2}}{\sigma}\left(\frac{3 A}{\delta^{4}} \delta^{\prime}\right) \\
-\frac{\alpha^{3 / 2}}{\sigma}\left(b \frac{C_{5}}{C_{4}} \frac{d T_{l v}}{d x}+\frac{\nu k_{l}}{C_{4} h_{f g}} \int_{0}^{x} \frac{T_{w}-T_{l v}}{\delta} d x\right)-\frac{b}{\sigma}\left(\frac{d T_{l v}}{d x}\right) \delta^{\prime \prime} \\
\delta^{\prime \prime \prime}=\frac{3 \delta^{\prime} \delta^{\prime \prime 2}}{\alpha}+\frac{\alpha^{3 / 2}}{\sigma}\left(\frac{3 A}{\delta^{4}} \delta^{\prime}-\frac{\nu k_{l}}{C_{4} h_{f g}} \int_{0}^{x} \frac{T_{w}-T_{l v}}{\delta} d x\right) \\
-\frac{d T_{l v}}{d x}\left(\frac{b}{\sigma}\right)\left(b \frac{C_{5}}{C_{4}} \alpha^{3 / 2}+\delta^{\prime \prime}\right)
\end{gathered}
$$

Let,

$$
\theta=\frac{\alpha^{3 / 2}}{\sigma}\left(\frac{3 A}{\delta^{4}} \delta^{\prime}-\frac{\nu k_{l}}{C_{4} h_{f g}} \int_{0}^{x} \frac{T_{w}-T_{l v}}{\delta} d x\right)
$$




$$
\psi=\left(\frac{b}{\sigma}\right)\left(\frac{C_{5}}{C_{4}} \alpha^{3 / 2}+\delta^{\prime \prime}\right)
$$

Thus,

$$
\delta^{\prime \prime \prime}=\frac{3 \delta^{\prime} \delta^{\prime 2}}{\alpha}+\theta-\psi \frac{d T_{l v}}{d x}
$$

Next to combine Equations (137) and (132).

$$
\begin{aligned}
\delta^{\prime \prime \prime} & =\frac{3 \delta^{\prime} \delta^{\prime 2}}{\alpha}+\theta-\psi\left(\frac{2 T_{v}}{\delta^{3}}\right)\left(\frac{\chi+V_{l} \eta \sigma \delta^{4} \kappa^{\prime}}{\omega}\right) \\
& =\frac{3 \delta^{\prime} \delta^{\prime \prime 2}}{\alpha}+\theta-\left(\frac{2 T_{v} \psi \chi}{\delta^{3} \omega}\right)-\left(\frac{2 T_{v} \psi V_{l} \eta \sigma \delta^{4} \kappa^{\prime}}{\omega \delta^{3}}\right) \\
& =\frac{3 \delta^{\prime} \delta^{\prime \prime 2}}{\alpha}+\theta-\left(\frac{2 T_{v} \psi \chi}{\delta^{3} \omega}\right)-\left(\frac{2 T_{v} \psi V_{l} \eta \sigma \delta}{\omega}\right) \kappa^{\prime}
\end{aligned}
$$

Combing Equations (138) and (73), we obtain the final third order differential equation.

$$
\delta^{\prime \prime \prime}=\frac{3 \delta^{\prime} \delta^{\prime 2}}{\alpha}+\theta-\left(\frac{2 T_{v} \psi \chi}{\delta^{3} \omega}\right)-\left(\frac{2 T_{v} \psi V_{l} \eta \sigma \delta}{\omega}\right)\left(\frac{\delta^{\prime \prime \prime}}{\alpha^{3 / 2}}-\frac{3 \delta^{\prime} \delta^{\prime 2}}{\alpha^{5 / 2}}\right)
$$

Next is the collecting of terms from Equation (139) to simplify the expression.

$$
\delta^{\prime \prime \prime}\left(1+\frac{2 T_{v} \psi V_{l} \eta \sigma \delta}{\omega \alpha^{3 / 2}}\right)=\frac{3 \delta^{\prime} \delta^{\prime \prime 2}}{\alpha}\left(1+\frac{2 T_{v} \psi V_{l} \eta \sigma \delta}{\omega \alpha^{3 / 2}}\right)+\theta-\frac{2 T_{v} \psi \chi}{\omega \delta^{3}}
$$

Let,

$$
\lambda=\left(1+\frac{2 T_{v} \psi V_{l} \eta \sigma \delta}{\omega \alpha^{3 / 2}}\right)
$$

Thus, the final form of the third order differential equation is:

$$
\delta^{\prime \prime \prime}=\frac{3 \delta^{\prime} \delta^{\prime 2}}{\alpha}-\frac{2 T_{v} \psi \chi}{\omega \delta^{3} \lambda}+\frac{\theta}{\lambda}
$$


Where,

$$
\begin{aligned}
& \sigma=a+b T_{l v} \\
& \alpha=1+\left(\delta^{\prime}\right)^{2} \\
& \eta=\left(\frac{C^{2} M}{2 \pi R}\right)^{1 / 2}\left(\frac{P_{v} h_{f g}}{R}\right) \\
& \kappa=\frac{\delta^{\prime \prime}}{\alpha^{3 / 2}} \\
& C_{4}=\frac{\delta^{3}}{3}-\beta \delta^{2} \\
& C_{5}=\frac{\delta^{2}}{2}-\beta \delta \\
& \chi=\delta^{\prime}\left[k_{l} \delta^{2}\left(T_{l v}^{5 / 2}-T_{w} T_{l v}^{3 / 2}\right)-3 A V_{l} \eta\right] \\
& \omega=k_{l} T_{v}\left(5 T_{l v}^{3 / 2}-3 T_{w} T_{l v}^{1 / 2}\right)+2 \delta \eta\left(M h_{f g}-V_{l} T_{v} b \kappa\right) \\
& \theta=\frac{\alpha^{3 / 2}}{\sigma}\left(\frac{3 A}{\delta^{4}} \delta^{\prime}-\frac{\nu k_{l}}{C_{4} h_{f g}} \int_{0}^{x} \frac{T_{w}-T_{l v}}{\delta} d x\right) \\
& \psi=\left(\frac{b}{\sigma}\right)\left(\frac{C_{5}}{C_{4}} \alpha^{3 / 2}+\delta^{\prime \prime}\right) \\
& \lambda=\left(1+\frac{2 T_{v} \psi V_{l} \eta \sigma \delta}{\omega \alpha^{3 / 2}}\right)
\end{aligned}
$$




\section{Appendix B}

\section{Determining the Adsorbed Film Thickness}

As the thin film progresses from the bulk region to the adsorbed region, the thickness asymptotically approaches a constant value. The region for which the film thickness is constant is considered the adsorbed thin film region. Within the adsorbed film, their is no evaporation or fluid flow. Setting the evaporative mass flux to zero would allow for the determination of the film thickness for a given superheat. This is achieved using the Kelvin-Clapeyron model,

$$
\dot{m}_{\text {evap }}^{\prime \prime}=\frac{\eta}{h_{f g}}\left(\frac{1}{T_{l v}}\right)^{3 / 2}\left[\frac{M h_{f g}}{T_{v}}\left(T_{l v}-T_{v}\right)-V_{l}\left(P_{d}+P_{c}\right)\right]=0
$$

The thin film thickness is of the order on nanometers and as such, the conductive losses are minimal. Thus the temperature at the interface of the adsorbed film is assumed to be,

$$
T_{l v} \approx T_{w}
$$

As the adsorbed layer of thin film is of constant thickness, the curvature must then be zero. Thus the capillary pressure within the adsorbed film is zero.

$$
P_{c}=\sigma \kappa \approx 0
$$


Lastly the evaporative mass flux in the thin film is nonexistent. This is due to the attractive van der Waals interaction between the thin film and the channel wall.

$$
\dot{m}_{\text {evap }}^{\prime \prime}=0
$$

Solving for the adsorbed film thickness,

$$
\begin{aligned}
0 & =\frac{\eta}{h_{f g}}\left(\frac{1}{T_{l v}}\right)^{3 / 2}\left[\frac{M h_{f g}}{T_{v}}\left(T_{w}-T_{v}\right)-V_{l} P_{d}\right] \\
V_{l} P_{d} & =\frac{M h_{f g}}{T_{v}}\left(T_{w}-T_{v}\right) \\
\frac{A}{\delta^{3}} & =\frac{M h_{f g}}{V_{l} T_{v}}\left(T_{w}-T_{v}\right) \\
\delta & =\left[\frac{A V_{l}}{M h_{f g}}\left(\frac{T_{v}}{T_{w}-T_{v}}\right)\right]^{1 / 3}
\end{aligned}
$$




\section{Appendix C}

\section{Second Derivative Limits}

The initial conditions which are applied to the third order ordinary differential equation, include $\delta, \delta^{\prime}$ and $\delta^{\prime \prime}$. The initial values assigned to the differential equation, are related in limitations via the evaporative mass flux balance. The evaporative mass flux balance comes from equating the Kelvin-Clapeyron model and the energy equation such that,

$$
\frac{k_{l}\left(T_{w}-T_{l v}\right)}{\delta h_{f g}}=\frac{\eta}{h_{f g}}\left(\frac{1}{T_{l v}}\right)^{3 / 2}\left[\frac{M h_{f g}}{T_{v}}\left(T_{l v}-T_{v}\right)-V_{l}\left(P_{d}+P_{c}\right)\right]
$$

The limits which may be imposed on the initial conditions stem from the selection of $\delta$ and $\delta^{\prime}$. The upper and lower limits are related by the extremes of the interface temperature. As such, the upper limit is associated with the wall temperature $\left(T_{w}\right)$ and the lower limit is associated with the vapour temperature $\left(T_{v}\right)$.

As discussed in literature [3], the initial conditions for film thickness and slope are sensitive to perturbations. Thus the limiting value is calculated for the $\delta^{\prime \prime}$ so as to avoid any solution divergences. 
Calculating the lower limit value for $\delta^{\prime \prime}$ :

Let $T_{l v} \approx T_{v}$ :

$$
\begin{aligned}
\frac{k_{l}\left(T_{w}-T_{v}\right)}{\delta h f g} & =\frac{\eta}{h_{f g}}\left(\frac{1}{T_{v}}\right)^{3 / 2}[\frac{M h_{f g}}{T_{v}} \underbrace{\left(T_{v}-T_{v}\right)}_{0}-V_{l}\left(P_{d}+P_{c}\right)] \\
-V_{l}\left(P_{d}+\sigma \kappa\right) & =\frac{k_{l}}{\delta \eta}\left(T_{v}\right)^{3 / 2}\left(T_{w}-T_{v}\right) \\
0 & =\frac{k_{l}}{\delta \eta}\left(T_{v}\right)^{3 / 2}\left(T_{w}-T_{v}\right)+V_{l} P_{d}+V_{l} \sigma \kappa \\
0 & =\frac{k_{l}\left(T_{v}\right)^{3 / 2}\left(T_{w}-T_{v}\right)+\delta \eta V_{l} P_{d}}{\delta \eta}+V_{l} \sigma\left(\frac{\delta^{\prime \prime}}{\alpha^{3 / 2}}\right) \\
0 & =\left(k_{l}\left(T_{v}\right)^{3 / 2}\left(T_{w}-T_{v}\right)+\delta \eta V_{l} P_{d}\right) \alpha^{3 / 2}+V_{l} \sigma \delta \eta \delta^{\prime \prime} \\
\delta^{\prime \prime} V_{l} \sigma \delta \eta & =-\alpha^{3 / 2}\left(k_{l}\left(T_{v}\right)^{3 / 2}\left(T_{w}-T_{v}\right)+\delta \eta V_{l} P_{d}\right) \\
\delta_{m i n}^{\prime \prime} & =-\alpha^{3 / 2}\left[\frac{k_{l}\left(T_{w}-T_{v}\right)}{V_{l} \sigma \delta \eta}\left(T_{v}\right)^{3 / 2}+\frac{P_{d}}{\sigma}\right]
\end{aligned}
$$

For the components of Equation (160), it is possible to determine if the overall total is greater or less than zero. This gives,

$$
\begin{gathered}
\frac{k_{l}\left(T_{w}-T_{v}\right)}{V_{l} \sigma \delta \eta}\left(T_{v}\right)^{3 / 2}>0 \\
\frac{P_{d}}{\sigma} \geq 0 \\
\alpha^{3 / 2}>0
\end{gathered}
$$

The negative multiplier in front of Equation (160) in conjunction with the conditions of Equations (161), (162) and (163) results in an overall negative limit for $\delta^{\prime \prime}$. Although this result in mathematically correct, the physics of the thin film limit the lower value of $\delta^{\prime \prime}$ to zero, as a negative value would suggest that the thin film would taper off into the channel wall and through it. 
Calculating the upper limit for $\delta^{\prime \prime}$,

Let $T_{l v} \approx T_{w}$ :

$$
\begin{aligned}
\frac{k_{l}\left(T_{w}-T_{w}\right)}{\delta h_{f g}} & =\frac{\eta}{h_{f g}}\left(\frac{1}{T_{v}}\right)^{3 / 2}\left[\frac{M h_{f g}}{T_{v}}\left(T_{w}-T_{v}\right)-V_{l}\left(P_{d}+P_{c}\right)\right] \\
0 & =\frac{M h_{f g}}{T_{v}}\left(T_{w}-T_{v}\right)-V_{l}\left(P_{d}+\sigma \kappa\right) \\
V_{l} P_{c} & =\frac{M h_{f g}}{T_{v}}\left(T_{w}-T_{v}\right)-V_{l} P_{d} \\
V_{l} \sigma\left(\frac{\delta^{\prime \prime}}{\alpha^{3 / 2}}\right) & =\frac{M h_{f g}}{T_{v}}\left(T_{w}-T_{v}\right)-V_{l} P_{d} \\
\delta_{\text {max }}^{\prime \prime} & =\frac{\alpha^{3 / 2}}{\sigma}\left(\frac{M h_{f g}}{T_{v} V_{l}}\left(T_{w}-T_{v}\right)-P_{d}\right)
\end{aligned}
$$

For the components of Equation (164), it is possible to determine if the overall total is greater or less than zero. This gives,

$$
\begin{gathered}
\frac{M h_{f g}}{T_{v} V_{l}}\left(T_{w}-T_{v}\right)>0 \\
P_{d} \geq 0 \\
\frac{\alpha^{3 / 2}}{\sigma} \geq 0
\end{gathered}
$$

Overall, Equation (164) can be either positive or negative. To ensure that $\delta^{\prime \prime}$ is greater than zero, the following condition must be satisfied.

$$
\left(\frac{M h_{f g}}{T_{v} V_{l}}-\frac{A}{\delta^{3}}\right) \geq 0
$$




\section{Appendix D}

\section{Reduction of Order}

The evaporating thin film portion of the meniscus, for the purposes of this analysis, was described in Appendix A by a third order differential equation. As a means of solving Equation (142), a system of first order differential equations can be defined, using the following change of variables:

$$
\begin{aligned}
& d_{0}=\delta \\
& d_{1}=\delta^{\prime} \\
& d_{2}=\delta^{\prime \prime} \\
& d_{2}^{\prime}=\delta^{\prime \prime \prime}
\end{aligned}
$$

Thus, the system of first order differential equations is given as:

$$
\begin{gathered}
d_{0}^{\prime}=d_{1} \\
d_{1}^{\prime}=d_{2} \\
d_{2}^{\prime}=\frac{3 d_{1} d_{2}^{2}}{\alpha}-\frac{2 T_{v} \psi \chi}{\omega \lambda d_{0}^{3}}+\frac{\theta}{\lambda}
\end{gathered}
$$


With the terms of Equation (175),

$$
\begin{gathered}
\sigma=a+b T_{l v} \\
\alpha=1+d_{1}^{2} \\
\eta=\left(\frac{C^{2} M}{2 \pi R}\right)^{1 / 2}\left(\frac{P_{v} h_{f g}}{R}\right) \\
\kappa=\frac{d_{2}}{\alpha^{3 / 2}} \\
C_{4}=\frac{d_{0}^{3}}{3}-\beta d_{0}^{2} \\
C_{5}=\frac{d_{0}^{2}}{2}-\beta d_{0} \\
\chi=d_{1}\left[k_{l} d_{0}^{2}\left(T_{l v}^{5 / 2}-T_{w} T_{l v}^{3 / 2}\right)-3 A V_{l} \eta\right] \\
\theta=\frac{\alpha^{3 / 2}}{\sigma}\left(\frac{3 A}{d_{0}^{4}} d_{1}-\frac{\nu k_{l}}{C_{4} h_{f g}} \int_{0}^{x} \frac{T_{w}-T_{l v}}{d_{0}} d x\right) \\
\psi=\left(\frac{b}{\sigma}\right)\left(\frac{C_{5}}{C_{4}} \alpha^{3 / 2}+d_{2}\right) \\
\lambda=\left(1+\frac{2 T_{v} \psi V_{l} \eta \sigma d_{0}}{\omega T_{l v}^{3 / 2}}\right)
\end{gathered}
$$

